\title{
THE SLOAN DIGITAL SKY SURVEY-II SUPERNOVA SURVEY: SEARCH ALGORITHM AND FOLLOW-UP OBSERVATIONS
}

\author{
Masao Sako $^{1,2}$, Bruce BassetT ${ }^{3,4}$, Andrew Becker ${ }^{5}$, David Cinabro ${ }^{6}$, Fritz DeJongh ${ }^{7}$, D. L. Depoy ${ }^{8}$, Ben Dilday ${ }^{9,10}$, \\ Mamoru Doi ${ }^{11}$, Joshua A. Frieman ${ }^{7,9,12}$, Peter M. Garnavich ${ }^{13}$, Craig J. Hogan ${ }^{14}$, Jon Holtzman ${ }^{15}$, Saurabh Jha $^{2}$, \\ Richard Kessler $^{9,16}$, Kohki Konishi ${ }^{17}$, Hubert Lampeitl ${ }^{18}$, John Marriner ${ }^{7}$, Gajus Miknaitis ${ }^{7}$, Robert C. Nichol ${ }^{19}$, \\ Jose Luis Prieto ${ }^{8}$, Adam G. Riess ${ }^{18,20}$, Michael W. Richmond ${ }^{21}$, Roger Romani ${ }^{2,22}$, Donald P. Schneider ${ }^{23}$,
} Mathew Smith ${ }^{19}$, Mark SubbaRao ${ }^{12}$, Naohiro Takanashi ${ }^{11}$, Kouichi Tokita ${ }^{11}$, Kurt van der Heyden $^{4}$, Naoki Yasuda ${ }^{17}$, Chen Zheng ${ }^{2,22}$, John Barentine ${ }^{24,25}$, Howard Brewington ${ }^{25}$, Changsu $\mathrm{CHOI}^{26}, \mathrm{~J}_{\text {ACK Dembicky }}{ }^{25}$, Michael HarnaveK ${ }^{25}$, Yutaka Ihara $^{27}$, Myungshin Im ${ }^{26}$, William Ketzeback ${ }^{25}$, Scott J. Kleinman ${ }^{25,28}$, Jurek KrZesiński ${ }^{25,29}$, Daniel C. Long ${ }^{25}$, Elena Malanushenko ${ }^{25}$, Viktor Malanushenko ${ }^{25}$, Russet J. McMillan ${ }^{25}$, Tomoki Morokuma ${ }^{27,30}$, Atsuko Nitta ${ }^{25,31}$, Kaike Pan ${ }^{25}$, Gabrelle Saurage ${ }^{25}$, and StePhanie A. Snedden ${ }^{25}$

${ }^{1}$ Department of Physics and Astronomy, University of Pennsylvania, 209 South 33rd Street, Philadelphia, PA 19104, USA

${ }^{2}$ Kavli Institute for Particle Astrophysics \& Cosmology, Stanford University, P.O. Box 20450, MS29, Stanford, CA 94309, USA

${ }^{3}$ Department of Mathematics and Applied Mathematics, University of Cape Town, Rondebosch 7701, South Africa

${ }^{4}$ South African Astronomical Observatory, P.O. Box 9, Observatory 7935, South Africa

${ }^{5}$ Department of Astronomy, University of Washington, Box 351580, Seattle, WA 98195, USA ${ }^{6}$ Department of Physics, Wayne State University, Detroit, MI 48202, USA

${ }^{7}$ Center for Particle Astrophysics, Fermi National Accelerator Laboratory, P.O. Box 500, Batavia, IL 60510, USA

${ }^{8}$ Department of Astronomy, Ohio State University, 140 West 18th Avenue, Columbus, OH 43210-1173, USA

${ }^{9}$ Kavli Institute for Cosmological Physics, The University of Chicago, 5640 South Ellis Avenue Chicago, IL 60637, USA

${ }^{10}$ Department of Physics, University of Chicago, Chicago, IL 60637, USA

${ }^{11}$ Institute of Astronomy, Graduate School of Science, University of Tokyo 2-21-1, Osawa, Mitaka, Tokyo 181-0015, Japan

12 Department of Astronomy and Astrophysics, The University of Chicago, 5640 South Ellis Avenue, Chicago, IL 60637, USA

${ }^{13}$ University of Notre Dame, 225 Nieuwland Science, Notre Dame, IN 46556-5670, USA

${ }^{14}$ Department of Astronomy, University of Washington, Box 351580, Seattle, WA 98195, USA

15 Department of Astronomy, MSC 4500, New Mexico State University, P.O. Box 30001, Las Cruces, NM 88003, USA

${ }^{16}$ Enrico Fermi Institute, University of Chicago, 5640 South Ellis Avenue, Chicago, IL 60637, USA

${ }^{17}$ Institute for Cosmic Ray Research, University of Tokyo, 5-1-5, Kashiwanoha, Kashiwa, Chiba, 277-8582, Japan

18 Space Telescope Science Institute, 3700 San Martin Drive, Baltimore, MD 21218, USA

${ }^{19}$ Institute of Cosmology and Gravitation, Mercantile House, Hampshire Terrace, University of Portsmouth, Portsmouth PO1 2EG, UK

${ }^{20}$ Department of Physics and Astronomy, Johns Hopkins University, 3400 North Charles Street, Baltimore, MD 21218, USA

${ }^{21}$ Physics Department, Rochester Institute of Technology, 85 Lomb Memorial Drive, Rochester, NY 14623-5603, USA ${ }^{22}$ Department of Physics, Stanford University, 382 Via Pueblo Mall, Stanford, CA 94305, USA

${ }^{23}$ Department of Astronomy and Astrophysics, The Pennsylvania State University, 525 Davey Laboratory, University Park, PA 16802, USA

${ }^{24}$ Department of Astronomy, McDonald Observatory, University of Texas, Austin, TX 78712, USA ${ }_{25}$ Apache Point Observatory, P.O. Box 59, Sunspot, NM 88349, USA

${ }^{26}$ Department of Astronomy, Seoul National University, Seoul, South Korea

${ }^{27}$ Institute of Astronomy, Graduate School of Science, University of Tokyo, 2-21-1, Osawa, Mitaka, Tokyo 181-0015, Japan ${ }^{28}$ Subaru Telescope, 650 North A'ohoku Place, Hilo, HI 96720, USA

${ }^{29}$ Obserwatorium Astronomiczne na Suhorze, Akademia Pedagogicazna w Krakowie, ulica Podchorążych 2, PL-30-084 Kraków, Poland

${ }^{30}$ National Observatory of Japan, 2-21-1, Osawa, Mitaka, Tokyo 181-8588, Japan

${ }^{31}$ Gemini Observatory, 670 North A'ohoku Place, Hilo, HI 96720, USA Received 2007 August 19; accepted 2007 September 27; published 2007 December 12

\begin{abstract}
The Sloan Digital Sky Survey-II Supernova Survey has identified a large number of new transient sources in a $300 \mathrm{deg}^{2}$ region along the celestial equator during its first two seasons of a three-season campaign. Multiband (ugriz) light curves were measured for most of the sources, which include solar system objects, galactic variable stars, active galactic nuclei, supernovae $(\mathrm{SNe})$, and other astronomical transients. The imaging survey is augmented by an extensive spectroscopic follow-up program to identify SNe, measure their redshifts, and study the physical conditions of the explosions and their environment through spectroscopic diagnostics. During the survey, light curves are rapidly evaluated to provide an initial photometric type of the SNe, and a selected sample of sources are targeted for spectroscopic observations. In the first two seasons, 476 sources were selected for spectroscopic observations, of which 403 were identified as SNe. For the type Ia SNe, the main driver for the survey, our photometric typing and targeting efficiency is $90 \%$. Only $6 \%$ of the photometric SN Ia candidates were spectroscopically classified as non-SN Ia instead, and the remaining $4 \%$ resulted in low signal-to-noise, unclassified spectra. This paper describes the search algorithm and the software, and the realtime processing of the SDSS imaging data. We also present the details of the supernova candidate selection procedures and strategies for follow-up spectroscopic and imaging observations of the discovered sources.
\end{abstract}

Key words: cosmology: observations - methods: data analysis - techniques: image processing - supernovae: general - surveys

Online-only material: color figures, machine-readable tables 


\section{INTRODUCTION}

Measurements of luminosity distances to type Ia supernovae ( $\mathrm{SNe}$ ) have played a central role in cosmology, leading two independent groups to the remarkable discovery of an unknown, currently-dominant component of the universe-dark energyand strong evidence for an accelerating universe (Riess et al. 1998; Perlmutter et al. 1999). Current surveys that target highredshift $\mathrm{SNe}$ from the ground-the Canada-France-Hawaii Telescope Supernova Legacy Survey $\left(\right.$ SNLS $^{32}$; Astier et al. 2006) and the Equation of State: Supernovae Trace Cosmic Expansion (ESSENCE ${ }^{33}$; Wood-Vasey et al. 2007; Miknaitis et al. 2007) — and from space using the Hubble Space Telescope (HST) (Riess et al. 2004a, 2007; Barbary et al. 2006) have substantially increased the sample of high- $z \mathrm{SNe}$, and have provided much-improved statistical constraints on the expansion history of the universe.

The discovery of cosmic acceleration was made possible in part through extensive observations of nearby type Ia $\mathrm{SNe}$ by the Calán/Tololo Supernova Search (Hamuy et al. 1993, 1996a, 1996b, 1996c) and by the CfA follow-up program (Riess et al. 1995; Riess 1996; Riess et al. 1999; Jha et al. 2006, 2007), and by studies pioneered by Pskovskii (1977) and by Phillips (1993) of the relationship between peak brightness and light curve decline rate (Hamuy et al. 1996d; Riess et al. 1996; Phillips et al. 1999). Current lowredshift SN surveys and follow-up programs (Lick Observatory Supernova Search (LOSS) $)^{34}$; Filippenko et al. 2001, Carnegie Supernova Project (CSP) ${ }^{35}$; Hamuy et al. 2006, Nearby Supernova Factory (SNFactory) ${ }^{36}$; Aldering et al. 2002, and the CfA $\mathrm{SN} \mathrm{Group}^{37}$ ) are continuing to discover $\mathrm{SNe}$ and compile a large number of high-quality multicolor light curves as well as multiepoch optical spectra of SNe Ia to expand the library of local training data used as "templates". These high-quality data sets will be indispensable for calibrating the brightness-decline relation to high precision. Obtaining and studying multi-epoch spectra are also important for computing improved $K$-corrections and minimizing systematic uncertainties (Kim et al. 1996; Nugent et al. 2002; Hsiao et al. 2007). Recent spectroscopic modeling efforts have also led to a better understanding of the physical mechanism responsible for the observed brightness-decline relation (see, e.g., Kasen \& Woosley 2007 and references therein).

As one of the three primary scientific components of the Sloan Digital Sky Survey-II (SDSS-II), the Supernova Survey takes repeated imaging scans of the same 300 square degrees of the sky during the fall seasons of 2005-7 to search for and measure light curves of SNe. The imaging survey is complemented by an extensive spectroscopic follow-up program to confirm the SN type and measure redshifts, and to study the detailed spectral properties of a sample of selected events.

This program exploits the unique capabilities of the SDSS $2.5 \mathrm{~m}$ telescope (Gunn et al. 2006) and its CCD imaging camera (Gunn et al. 1998) to survey a large volume of space at moderately high cadence. The survey complements and improves upon other low $z$ and high- $z$ surveys in several important ways. The wide-field-of-view camera operating in

\footnotetext{
32 http://www.cfht.hawaii.edu/SNLS.

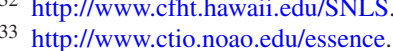

$34 \mathrm{http} / / /$ astro.berkeley.edu/bait/kait.html.

35 http://csp1.lco.cl/cspuser1/PUB/CSP.html.

$36 \mathrm{http}: / /$ snfactory.lbl.gov/.

37 http://cfa-www.harvard.edu/oir/Research/supernova/SNgroup.html
}

drift scan mode allows for efficient discoveries of type Ia $\mathrm{SNe}$ at $0.05 \lesssim z \lesssim 0.4$, a redshift interval that is not easily probed by other existing surveys that target either known nearby galaxies $($ low $-z$ ) or narrow pencil-beam volumes (high- $z$ ). Its well-calibrated multi-band photometric system (ugriz; Fukugita et al. 1996) enables precise measurements of supernova light curves with controlled systematics. The absolute magnitude scale is accurate to better than $\sim 2 \%$ in $r$ and $\sim 2-3 \%$ in the colors (Adelman-McCarthy et al. 2007), and a factor of $\sim 2$ improvement has been obtained from repeat imaging of the equatorial region (Ivezić et al. 2007). Finally, the survey is sensitive to the redshift interval that, given a large enough sample, enables cosmological distance measurements with data from a single telescope, eliminating the need for crosscalibration across two or more photometric systems.

This paper is part of a series describing the SDSS-II Supernova Survey. Here we present a technical description of the search algorithm, data processing, photometric typing of SN candidates, and spectroscopic target selection. Frieman et al. (2007) present an overview of the program. Photometry and light curves of the full sample of spectroscopically confirmed SNe from the 2005 season are presented in Holtzman et al. (2007). Spectroscopic data and their analysis results are described in Zheng et al. (2007). Kessler et al. (2007) present the SN Ia Hubble diagram and cosmological analysis from the 2005 season. The measurement of the low-redshift type Ia $\mathrm{SN}$ rate is presented in Dilday et al. (2007). Detailed studies of two peculiar SNe discovered by the SDSS-II SN Survey, SN2005hk and SN2005gj, are presented in Phillips et al. (2007) and Prieto et al. (2007), respectively.

The main body of the paper is separated into two broad sections. The first part (Section 2) presents the details of the real-time on-mountain data processing and the mechanics of the search pipeline, which identifies new transient events. The second part (Section 3) describes the procedures for supernova candidate identification, photometric SN typing, and the algorithm adopted for selecting targets for spectroscopy. A brief discussion of follow-up imaging observations of a sample of spectroscopically confirmed $\mathrm{SNe}$ is presented in Section 4. The general results from the 2005 and 2006 seasons are presented in Section 5. We briefly summarize in Section 6.

\section{SDSS 2.5 M OBSERVATIONS AND DATA PROCESSING}

The SDSS-II Supernova Survey has been allocated the bulk of the time during the fall seasons (September 1-November 30) of 2005-7 on the SDSS telescope at Apache Point Observatory (APO). Imaging observations are scheduled on most nights excluding a 5-day period around full moon. Some nights are shared with the SEGUE program especially during stretches of consecutive nights with good observing conditions. An overview of the SDSS is given by York et al. (2000); see Frieman et al. (2007) for an overview of the SN Survey.

\subsection{Survey Area: Stripe 82}

The survey area is Stripe 82 , the $300 \mathrm{deg}^{2}$ southern equatorial stripe of the SDSS footprint, which covers the approximate coordinate ranges $-60^{\circ} \lesssim \alpha \lesssim+60^{\circ}(20 \mathrm{~h}$ to $4 \mathrm{~h}$ in right ascension, R.A. or $\alpha$ ) and $-1.25^{\circ} \lesssim \delta \lesssim+1.25^{\circ}$ in declination $(\delta)$. The detailed Stripe 82 footprint of each camera column is shown in Table 1. This survey area was selected for three primary reasons: (1) extensive repeat observations were acquired as part of the SDSS-I survey, before the start 
Table 1

Stripe 82 Footprint

\begin{tabular}{lccr}
\hline \hline Strip & $\begin{array}{c}\text { Camera } \\
\text { column }\end{array}$ & $\delta_{\min }{ }^{\mathrm{a}}$ & $\delta_{\max }{ }^{\mathrm{a}}$ \\
\hline $82 \mathrm{~N}$ & 1 & $-1.058^{\circ}$ & $-0.833^{\circ}$ \\
$\ldots$ & 2 & $-0.638^{\circ}$ & $-0.413^{\circ}$ \\
$\ldots$ & 3 & $-0.219^{\circ}$ & $+0.007^{\circ}$ \\
$\ldots$ & 4 & $+0.201^{\circ}$ & $+0.426^{\circ}$ \\
$\ldots$ & 5 & $+0.621^{\circ}$ & $+0.846^{\circ}$ \\
$\ldots$ & 6 & $+1.041^{\circ}$ & $+1.266^{\circ}$ \\
$82 \mathrm{~S}$ & 1 & $-1.267^{\circ}$ & $-1.042^{\circ}$ \\
$\ldots$ & 2 & $-0.848^{\circ}$ & $-0.622^{\circ}$ \\
$\ldots$ & 3 & $-0.428^{\circ}$ & $-0.203^{\circ}$ \\
$\ldots$ & 4 & $-0.008^{\circ}$ & $+0.217^{\circ}$ \\
$\ldots$ & 5 & $+0.411^{\circ}$ & $+0.637^{\circ}$ \\
$\ldots$ & 6 & $+0.831^{\circ}$ & $+1.057^{\circ}$ \\
\hline & & &
\end{tabular}

Note. ${ }^{\text {a }}$ These values correspond to the nominal $\delta$ values. The exact telescope pointing differs slightly from night to night resulting in a shift in $\delta$ by as much as $0.003^{\circ}$ in either direction.

of the SN Survey, (2) the area is easily accessible to most telescopes, and (3) it is accessible at low airmass during the fall months, when most of the northern SDSS area is not. The advantages of having repeat observations from SDSS-I are three-fold. First, the data provide a catalog of known variable sources, which is crucial for distinguishing a small number of new SN candidates from a large population of foreground variable stars and background active galactic nuclei. Second, the deep co-added images constructed from the individual scans serve as references for image subtraction, enabling a more sensitive search of new transient events (the template is essentially noiseless in the detection process). Finally, the deep co-adds also allow identification of faint host galaxies that are otherwise undetected in the single-scan images, which are frequently useful for prioritizing follow-up observations. The stripe contains over three million cataloged galaxies that are brighter than $r \sim 22.5 \mathrm{mag}$.

Due to gaps between the six CCD columns, the SDSS imaging camera is capable of scanning approximately half of the stripe (or one strip) in a single night. During the 2005 season, the observations alternated between the northern strip $(82 \mathrm{~N})$ and the southern strip (82S) from night to night. In 2006, however, a significant effort was made to avoid long temporal gaps in any given part of the strip, so pieces of R.A. ranges from both strips were sometimes observed in a single night. We also note that approximately $10 \%$ of Stripe 82 is covered by both the northern and southern strips due to overlapping CCD columns, so $\sim 30 \mathrm{deg}^{2}$ of the sky is observed on a cadence of 1 day (modulo weather losses). The lists of all SDSS-II SN runs, i.e., continuous imaging scans, and their corresponding R.A. ranges taken during the 2005 and 2006 seasons are given in Tables 2 and 3, respectively. We also show in Figure 1 the number of visits made to each of the strips in 2005 and 2006 as a function of R.A. The complete set of corrected frames and the uncalibrated object catalogs from 2005 and 2006 are available online as part of the first $\mathrm{SN}$ data release $\left(\mathrm{DRSN1}{ }^{38}\right)$. These data are also accessible from the Data Archive Server of the Sixth Data Release $^{39}$ (Adelman-McCarthy et al. 2007).

\footnotetext{
38 http://www.sdss.org/drsn1/DRSN1_data_release.html.

39 http://www.sdss.org/dr6/access/index.html.
}

Table 2

2005 SDSS Supernova Imaging Runs

\begin{tabular}{|c|c|c|c|c|c|}
\hline Run & Strip & MJD $_{\text {start }}$ & MJD $_{\text {end }}$ & R.A.start & R.A.end \\
\hline 5566 & $\mathrm{~N}$ & 53616.215 & 53616.469 & -32.72 & +58.91 \\
\hline 5582 & $\mathrm{~S}$ & 53622.207 & 53622.477 & -40.36 & +57.41 \\
\hline 5590 & $\mathrm{~N}$ & 53623.156 & 53623.324 & -50.67 & +9.96 \\
\hline 5597 & $\mathrm{~S}$ & 53625.176 & 53625.266 & -50.62 & -18.43 \\
\hline 5603 & $\mathrm{~N}$ & 53626.141 & 53626.449 & -50.62 & +60.17 \\
\hline 5607 & $\mathrm{~S}$ & 53627.137 & 53627.441 & -50.59 & +59.90 \\
\hline 5610 & $\mathrm{~N}$ & 53628.129 & 53628.434 & -50.57 & +60.21 \\
\hline 5619 & $\mathrm{~S}$ & 53634.129 & 53634.438 & -50.68 & +60.71 \\
\hline 5622 & $\mathrm{~N}$ & 53635.137 & 53635.445 & -50.72 & +60.66 \\
\hline 5628 & $\mathrm{~S}$ & 53636.133 & 53636.281 & -50.70 & +1.99 \\
\hline 5633 & $\mathrm{~N}$ & 53637.129 & 53637.371 & -50.68 & +36.45 \\
\hline 5637 & $\mathrm{~S}$ & 53638.2 & 53638.477 & -20.72 & +60.72 \\
\hline 5642 & $\mathrm{~N}$ & 53639.289 & 53639.484 & -9.82 & +60.69 \\
\hline 5646 & $\mathrm{~S}$ & 53640.234 & 53640.441 & -13.85 & +60.70 \\
\hline 5654 & $\mathrm{~N}$ & 53641.113 & 53641.121 & -55.42 & -53.02 \\
\hline 5658 & $\mathrm{~N}$ & 53641.359 & 53641.465 & +16.08 & +54.56 \\
\hline 5666 & $\mathrm{~S}$ & 53643.430 & 53643.484 & +41.25 & +60.71 \\
\hline 5670 & $\mathrm{~N}$ & 53644.109 & 53644.430 & -55.53 & +60.79 \\
\hline 5675 & $\mathrm{~S}$ & 53645.180 & 53645.219 & -55.52 & -41.75 \\
\hline 5681 & $\mathrm{~S}$ & 53646.418 & 53646.480 & +29.97 & +52.88 \\
\hline 5698 & $\mathrm{~S}$ & 53648.164 & 53648.289 & -55.62 & -9.81 \\
\hline 5702 & $\mathrm{~N}$ & 53649.102 & 53649.129 & -55.55 & -46.42 \\
\hline 5709 & $\mathrm{~N}$ & 53654.152 & 53654.359 & -51.60 & +23.25 \\
\hline 5713 & $\mathrm{~S}$ & 53655.094 & 53655.363 & -55 & +41.47 \\
\hline 5719 & $\mathrm{~N}$ & 53656.090 & 53656.246 & -5 & +1.14 \\
\hline 5729 & $\mathrm{~S}$ & 53657.203 & 53657.2 & -46 & -43.05 \\
\hline 5730 & $\mathrm{~S}$ & 53657.258 & 53657.324 & +37 & +60.71 \\
\hline 5731 & $\mathrm{~N}$ & 53657.332 & 53657.441 & +21.17 & +60.69 \\
\hline 5732 & $\mathrm{~S}$ & 53657.449 & 53657.488 & +47.11 & +60.73 \\
\hline 5743 & $\mathrm{~N}$ & 53663.086 & 53663.164 & -55.42 & -26.98 \\
\hline 5744 & $\mathrm{~N}$ & 53663.176 & 53663.418 & -29.75 & +57.83 \\
\hline 5745 & $\mathrm{~S}$ & 53663.430 & 53663.488 & +33.09 & +55.10 \\
\hline 5754 & $\mathrm{~S}$ & 53664.117 & 53664.367 & -55.60 & +34.37 \\
\hline 5759 & $\mathrm{~N}$ & 53665.082 & 53665.395 & -55.52 & +57.81 \\
\hline 5760 & $\mathrm{~S}$ & 53665.406 & 53665.492 & +25.13 & +55.68 \\
\hline 5763 & $\mathrm{~S}$ & 53666.082 & 53666.246 & -55.49 & +3.94 \\
\hline 5765 & $\mathrm{~N}$ & 53666.332 & 53666.480 & +2.30 & +54.86 \\
\hline 5770 & $\mathrm{~N}$ & 53668.094 & 53668.406 & -55 & +57.94 \\
\hline 5771 & $\mathrm{~S}$ & 53668.418 & 8.492 & +33 & +6 \\
\hline 5776 & $\mathrm{~S}$ & 53669.078 & 53669.391 & -55 & +57.82 \\
\hline 5777 & $\mathrm{~N}$ & 53669.406 & 53669.496 & +25 & +57.96 \\
\hline 5781 & $\mathrm{~N}$ & 53670.098 & 53670.410 & -55 . & +57.86 \\
\hline 5782 & $\mathrm{~S}$ & 53670.422 & 53670.500 & +32.65 & +60.66 \\
\hline 5786 & $\mathrm{~S}$ & 53671.273 & 53671.484 & -14.82 & +60.65 \\
\hline 5792 & $\mathrm{~N}$ & 53673.066 & 53673.379 & -55.42 & +57.91 \\
\hline 5797 & $\mathrm{~S}$ & 53674.062 & 53674.375 & -55.57 & +58.05 \\
\hline 5800 & $\mathrm{~N}$ & 53675.062 & 53675.371 & -55.61 & +56.07 \\
\hline 5807 & $\mathrm{~S}$ & 53676.172 & 53676.465 & -47.67 & +57.89 \\
\hline 5808 & $\mathrm{~N}$ & 53676.473 & 53676.496 & +49.27 & +57.96 \\
\hline 5813 & $\mathrm{~N}$ & 53677.082 & 53677.359 & -55.56 & +44.59 \\
\hline 5820 & $\mathrm{~S}$ & 53679.152 & 53679.441 & -44.54 & +59.96 \\
\hline 5823 & $\mathrm{~N}$ & 53680.070 & 53680.391 & -55.46 & +60.71 \\
\hline 5836 & $\mathrm{~S}$ & 53681.113 & 53681.438 & -55.48 & +60.70 \\
\hline 5842 & $\mathrm{~N}$ & 53683.129 & 53683.449 & -55.43 & +60.76 \\
\hline 5847 & $\mathrm{~S}$ & 53684.301 & 53684.398 & +25.71 & +60.74 \\
\hline 5853 & $\mathrm{~S}$ & 53685.062 & 53685.172 & -58.95 & -19.28 \\
\hline 5864 & $\mathrm{~N}$ & 53686.141 & 53686.152 & -50.06 & -45.42 \\
\hline 5865 & $\mathrm{~N}$ & 53686.172 & 53686.242 & -42.96 & -18.11 \\
\hline 5866 & $\mathrm{~N}$ & 53686.273 & 53686.391 & +18.21 & +60.73 \\
\hline 5870 & $\mathrm{~S}$ & 53687.109 & 53687.250 & -55.57 & -6.16 \\
\hline 5871 & $\mathrm{~S}$ & 53687.258 & 53687.340 & +31.39 & +60.74 \\
\hline 5872 & $\mathrm{~N}$ & 53687.352 & 53687.449 & +25.14 & +60.78 \\
\hline 5878 & $\mathrm{~N}$ & 53693.059 & 53693.395 & -59.98 & +60.68 \\
\hline 5882 & $\mathrm{~S}$ & 53694.074 & 53694.410 & -60.11 & +60.71 \\
\hline 5889 & $\mathrm{~S}$ & 53696.355 & 53696.402 & +44.01 & +60.78 \\
\hline
\end{tabular}


Table 2

(Continued)

\begin{tabular}{lccccc}
\hline \hline Run & Strip & MJD $_{\text {start }}$ & MJD $_{\text {end }}$ & R.A.start & R.A.end \\
\hline 5895 & S & 53697.059 & 53697.395 & -60.11 & +60.71 \\
5898 & N & 53698.059 & 53698.129 & -59.98 & -33.63 \\
5902 & N & 53699.082 & 53699.301 & -60.13 & +18.78 \\
5905 & S & 53700.059 & 53700.391 & -59.99 & +60.69 \\
5909 & N & 53702.059 & 53702.211 & -59.35 & -4.10 \\
5915 & N & 53703.066 & 53703.156 & -60.01 & -27.07 \\
5918 & N & 53704.059 & 53704.379 & -54.46 & +60.67 \\
5924 & S & 53705.043 & 53705.379 & -60.06 & +60.76 \\
\hline
\end{tabular}

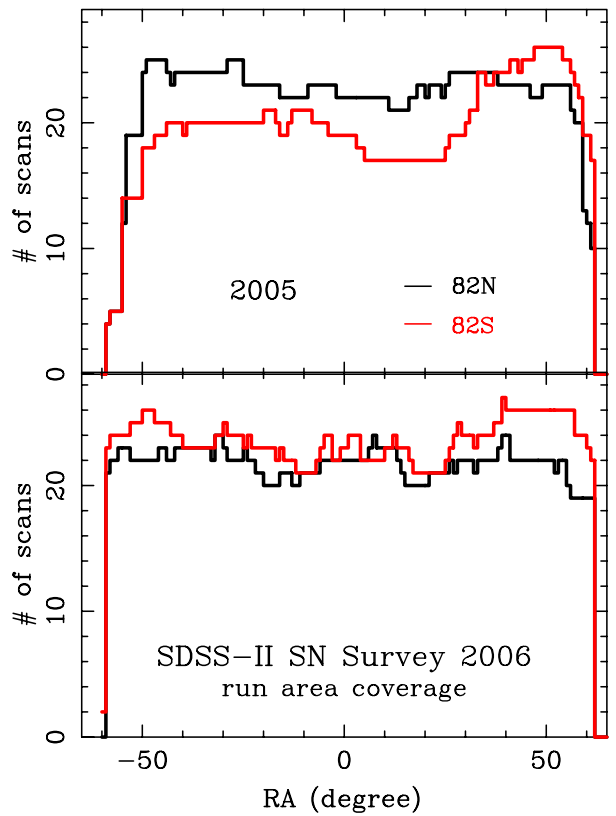

Figure 1. Number of imaging scans of the northern (black) and southern (red) strips for the 2005 (top) and 2006 (bottom) seasons as a function of right ascension. Note that, in contrast to the 2005 season, the 2006 scans are more evenly distributed in R.A. with very little difference between the northern and southern strips.

(A color version of this figure is available in the online journal)

\subsection{On-Mountain Processing}

The SDSS $2.5 \mathrm{~m}$ telescope nominally performs observations every night during the search seasons, so the data must be processed within $24 \mathrm{~h}$ to avoid a backlog of unprocessed data and to rapidly identify SN candidates for spectroscopic followup observations. A single night of observing produces up to $\sim 200 \mathrm{~Gb}$ of imaging data, so it is highly desirable to process the data on the mountain and significantly reduce the number of images that are transferred over the internet for visual inspection.

The data are processed on a dedicated 10 dual-processor (20 processors total) computer cluster that runs at APO. There are 9 computers that process data; each of the 18 processors is responsible for processing all images from a single combination of filter ( $\mathrm{gri}$ ) and camera column (1-6). Each computer has $400 \mathrm{~Gb}$ of RAID5 disk and $2 \mathrm{~Gb}$ memory. The tenth monitor/backup computer has $3 \mathrm{~Tb}$ of disk and $4 \mathrm{~Gb}$ memory, and is used for process-management, data transfer, and software development. This setup was specifically chosen to meet our requirement of processing a full night's worth of data within $24 \mathrm{~h}$. An 11th computer is available as a spare but so far has not been needed-during the first two years of operation, there were

Table 3

2006 SDSS Supernova Imaging Runs

\begin{tabular}{|c|c|c|c|c|c|}
\hline Run & Strip & MJD $_{\text {start }}$ & MJD $_{\text {end }}$ & R.A.start & R.A.end \\
\hline 6281 & $\mathrm{~N}$ & 53974.141 & 53974.203 & -56.82 & -34.52 \\
\hline 6283 & $\mathrm{~N}$ & 53974.270 & 53974.336 & -11.69 & +12.42 \\
\hline 6287 & $\mathrm{~S}$ & 53975.133 & 53975.465 & -58.78 & +60.70 \\
\hline 6293 & $\mathrm{~N}$ & 53977.371 & 53977.387 & +7.31 & +13.45 \\
\hline 6313 & $\mathrm{~N}$ & 53989.121 & 53989.160 & -60.05 & -45.99 \\
\hline 6314 & $\mathrm{~N}$ & 53989.176 & 53989.473 & -47.39 & +60.70 \\
\hline 6330 & $\mathrm{~S}$ & 53990.312 & 53990.387 & +1.17 & +27.82 \\
\hline 6348 & $\mathrm{~S}$ & 53993.277 & 53993.371 & -31.19 & +2.94 \\
\hline 6349 & $\mathrm{~S}$ & 53993.379 & 53993.414 & +26.11 & +38.98 \\
\hline 6353 & $\mathrm{~S}$ & 53994.125 & 53994.156 & -59.99 & -48.77 \\
\hline 6355 & $\mathrm{~S}$ & 53994.301 & 53994.484 & -5.78 & +59.80 \\
\hline 6360 & $\mathrm{~N}$ & 53995.129 & 53995.254 & -59.98 & -14.92 \\
\hline 6362 & $\mathrm{~N}$ & 53995.344 & 53995.391 & +9.13 & +25.90 \\
\hline 6363 & $\mathrm{~N}$ & 53995.414 & 53995.453 & +25.67 & +40.04 \\
\hline 6367 & $\mathrm{~S}$ & 53996.168 & 53996.469 & -47.82 & +60.72 \\
\hline 6370 & $\mathrm{~N}$ & 53997.105 & 53997.199 & -59.99 & -26.15 \\
\hline 6373 & $\mathrm{~N}$ & 53997.352 & 53997.422 & -17.22 & +8.09 \\
\hline 6374 & $\mathrm{~N}$ & 53997.434 & 53997.469 & +38.16 & +50.88 \\
\hline 6377 & $\mathrm{~S}$ & 53998.105 & 53998.312 & -60.00 & +14.85 \\
\hline 6383 & $\mathrm{~N}$ & 54000.191 & 54000.430 & -32.88 & +53.50 \\
\hline 6391 & $\mathrm{~N}$ & 54003.145 & 54003.160 & -60.06 & -54.52 \\
\hline 6400 & $\mathrm{~N}$ & 54005.078 & 54005.160 & -60.02 & -30.98 \\
\hline 6401 & $\mathrm{~S}$ & 54005.168 & 54005.191 & -50.90 & -41.62 \\
\hline 6402 & $\mathrm{~S}$ & 54005.230 & 54005.332 & -24.92 & +11.76 \\
\hline 6404 & $\mathrm{~S}$ & 54005.383 & 54005.484 & +24.97 & +60.75 \\
\hline 6408 & $\mathrm{~S}$ & 54006.094 & 54006.125 & -59.99 & -48.77 \\
\hline 6409 & $\mathrm{~N}$ & 54006.137 & 54006.395 & -32.79 & +60.78 \\
\hline 6412 & $\mathrm{~N}$ & 54007.094 & 54007.191 & -58.91 & -24.18 \\
\hline 6414 & $\mathrm{~N}$ & 54007.258 & 54007.484 & -25.93 & +54.91 \\
\hline 6417 & $\mathrm{~S}$ & 54008.094 & 54008.430 & -60.13 & +60.68 \\
\hline 6418 & $\mathrm{~N}$ & 54008.438 & 54008.461 & +53.21 & +60.70 \\
\hline 6421 & $\mathrm{~N}$ & 54009.090 & 54009.422 & -60.12 & +60.69 \\
\hline 6422 & $\mathrm{~S}$ & 54009.434 & 54009.484 & +37.09 & +56.10 \\
\hline 6425 & $\mathrm{~S}$ & 54010.090 & 54010.426 & -60.04 & +60.77 \\
\hline 6430 & $\mathrm{~N}$ & 54011.152 & 54011.488 & -60.10 & +60.71 \\
\hline 6433 & $\mathrm{~S}$ & 54012.082 & 54012.418 & -60.08 & +60.73 \\
\hline 6435 & $\mathrm{~N}$ & 54012.434 & 54012.484 & +36.09 & +54.35 \\
\hline 6441 & $\mathrm{~N}$ & 54019.102 & 54019.289 & -60.13 & +6.64 \\
\hline 6444 & $\mathrm{~N}$ & 54019.371 & 54019.480 & +21.13 & +60.66 \\
\hline 6447 & $\mathrm{~S}$ & 54020.090 & 54020.367 & -60.05 & +40.10 \\
\hline 6448 & $\mathrm{~S}$ & 54020.395 & 54020.457 & +39.08 & +60.79 \\
\hline 6450 & $\mathrm{~N}$ & 54021.305 & 54021.492 & -6.74 & +60.79 \\
\hline 6453 & $\mathrm{~S}$ & 54022.137 & 54022.293 & -60.01 & -4.47 \\
\hline 6458 & $\mathrm{~S}$ & 54024.316 & 54024.492 & -6.73 & +55.85 \\
\hline 6461 & $\mathrm{~N}$ & 54025.152 & 54025.488 & -60.11 & +60.71 \\
\hline 6464 & $\mathrm{~S}$ & 54026.090 & 54026.133 & -59.99 & -45.02 \\
\hline 6468 & $\mathrm{~S}$ & 54028.078 & 54028.254 & -60.06 & +2.96 \\
\hline 6471 & $\mathrm{~S}$ & 54028.352 & 54028.492 & +9.53 & +60.74 \\
\hline 6474 & $\mathrm{~N}$ & 54029.082 & 54029.336 & -60.05 & +31.41 \\
\hline 6476 & $\mathrm{~N}$ & 54029.414 & 54029.496 & +30.56 & +60.66 \\
\hline 6479 & $\mathrm{~S}$ & 54030.082 & 54030.293 & -60.09 & +15.96 \\
\hline 6480 & $\mathrm{~S}$ & 54030.371 & 54030.445 & +33.19 & +59.24 \\
\hline 6484 & $\mathrm{~N}$ & 54031.070 & 54031.402 & -60.01 & +60.66 \\
\hline 6488 & $\mathrm{~S}$ & 54032.070 & 54032.164 & -60.06 & -26.83 \\
\hline 6494 & $\mathrm{~S}$ & 54034.137 & 54034.223 & -61.51 & -31.27 \\
\hline 6501 & $\mathrm{~S}$ & 54035.234 & 54035.492 & -32.56 & +60.72 \\
\hline 6504 & $\mathrm{~N}$ & 54036.074 & 54036.410 & -60.00 & +60.67 \\
\hline 6508 & $\mathrm{~S}$ & 54037.062 & 54037.398 & -60.52 & +60.59 \\
\hline 6513 & $\mathrm{~N}$ & 54039.070 & 54039.406 & -60.08 & +60.73 \\
\hline 6518 & $\mathrm{~S}$ & 54040.078 & 54040.414 & -60.12 & +60.69 \\
\hline 6522 & $\mathrm{~N}$ & 54041.074 & 54041.152 & -60.00 & -31.86 \\
\hline 6524 & $\mathrm{~N}$ & 54041.227 & 54041.379 & +6.11 & +60.75 \\
\hline 6525 & $\mathrm{~S}$ & 54041.387 & 54041.449 & +39.12 & +60.68 \\
\hline 6528 & $\mathrm{~S}$ & 54047.070 & 54047.164 & -60.11 & -25.23 \\
\hline 6530 & $\mathrm{~S}$ & 54047.254 & 54047.441 & -7.98 & +60.74 \\
\hline
\end{tabular}


Table 3

(Continued)

\begin{tabular}{lccccc}
\hline \hline Run & Strip & MJD $_{\text {start }}$ & MJD $_{\text {end }}$ & R.A.start & R.A.end \\
\hline 6533 & $\mathrm{~N}$ & 54048.066 & 54048.344 & -60.10 & +39.61 \\
6534 & $\mathrm{~N}$ & 54048.367 & 54048.430 & +38.70 & +60.71 \\
6537 & $\mathrm{~S}$ & 54049.223 & 54049.438 & -17.38 & +60.78 \\
6542 & $\mathrm{~S}$ & 54050.062 & 54050.215 & -59.99 & -4.60 \\
6545 & $\mathrm{~S}$ & 54050.344 & 54050.398 & +41.22 & +60.68 \\
6548 & $\mathrm{~N}$ & 54051.059 & 54051.164 & -60.03 & -22.01 \\
6552 & $\mathrm{~N}$ & 54052.070 & 54052.406 & -60.05 & +60.77 \\
6555 & $\mathrm{~S}$ & 54053.070 & 54053.320 & -60.02 & +31.00 \\
6556 & $\mathrm{~S}$ & 54053.328 & 54053.418 & +28.37 & +60.71 \\
6559 & $\mathrm{~N}$ & 54054.090 & 54054.293 & -60.06 & +14.05 \\
6564 & $\mathrm{~N}$ & 54055.066 & 54055.316 & -60.10 & +30.18 \\
6565 & $\mathrm{~N}$ & 54055.328 & 54055.418 & +28.17 & +60.66 \\
6568 & $\mathrm{~S}$ & 54056.070 & 54056.406 & -60.05 & +60.77 \\
6571 & $\mathrm{~S}$ & 54057.055 & 54057.152 & -53.76 & -19.32 \\
6577 & $\mathrm{~N}$ & 54058.066 & 54058.398 & -59.99 & +60.68 \\
6580 & $\mathrm{~S}$ & 54059.066 & 54059.398 & -60.06 & +60.76 \\
6584 & $\mathrm{~N}$ & 54060.137 & 54060.422 & -42.83 & +60.78 \\
6590 & $\mathrm{~S}$ & 54061.074 & 54061.203 & -60.09 & -13.52 \\
6592 & $\mathrm{~S}$ & 54061.277 & 54061.410 & +12.07 & +60.73 \\
6596 & $\mathrm{~S}$ & 54062.070 & 54062.402 & -60.07 & +60.75 \\
6600 & $\mathrm{~N}$ & 54063.055 & 54063.320 & -60.10 & +34.82 \\
6604 & $\mathrm{~S}$ & 54064.309 & 54064.402 & +27.24 & +60.78 \\
6609 & $\mathrm{~N}$ & 54065.320 & 54065.398 & +33.18 & +60.73 \\
6615 & $\mathrm{~S}$ & 54068.059 & 54068.180 & -60.05 & -16.78 \\
6618 & $\mathrm{~S}$ & 54068.211 & 54068.387 & -1.81 & +60.77 \\
\hline & & & & &
\end{tabular}

no serious failures. Several disks failed, but the RAID system worked properly to prevent any loss of processing time while waiting for a disk to be replaced. There was one curious glitch that may be related to operation at high altitude $(2800 \mathrm{~m})$. On occasion one of the computers would hang up, and cycling the power was the only way to revive the system. There were about 20 such incidents per season, although the probability seemed higher in the month of November, possibly due to better weather and longer nights resulting in more computing time. Since people are present at APO day and night, minimal processing time was lost from these hang-ups.

There are three main tasks for the process manager: (i) start jobs, (ii) monitor processing progress, and (iii) monitor disk space. Due to the structure of the data acquisition system, the image processing can start only after an imaging run has finished, and the master script is usually executed manually by a person in the morning. While this script could have been automatically started, it is better that a human operator reviews the observer logs and checks that processing starts smoothly. The master script schedules and allocates resources for copying data from the data acquisition computer to a local disk, photometric reduction in ugriz (Section 2.2.1), and frame subtraction (Section 2.2.2) and object detection (Section 2.2.3) in gri. Several monitor scripts are used to check the status throughout the $\sim 20 \mathrm{~h}$ needed to process the data. Since data processing continues virtually round-the-clock, a few people often shared the monitoring burden. Continuous monitoring was necessary because there were two common problems that could interupt the processing. First, poor observing conditions caused the photometric reduction software to abort. In this case, the photometric reduction must be reprocessed with a more restrictive R.A. range. The second source of interruption was the computer hang-ups discussed above. The last process-manager issue concerns disk space. Rather than clearing disk space after each night's data were processed, we kept all of the subtracted

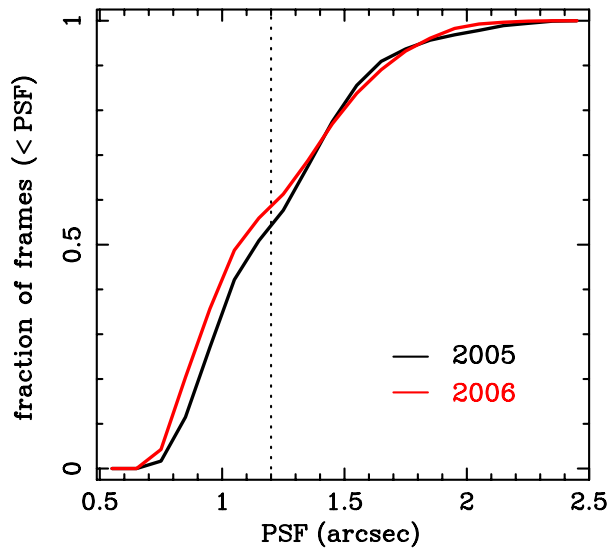

Figure 2. Cumulative distributions of the $r$-band PSF during the 2005 (black) and 2006 (red) search seasons. More than half of the frames were acquired at $<1.2^{\prime \prime}$. Only frames successfully processed through Photo are included.

(A color version of this figure is available in the online journal)

images at APO for 1-2 weeks. The reason for keeping subtracted images is that for interesting candidates we could go back to earlier epochs and process $u$ and $z$ : the $u$ band was particularly useful for distinguishing type Ia and type II supernovae. In addition, since the candidate position was known, we re-ran gri photometry at the known location in previous epochs to get a better estimate of the early-epoch fluxes and upper limits. The disk space was managed with a priority system so that clearing the disk was mostly based on following a pre-defined algorithm and rarely involved a hasty decision. To avoid risks with automated disk-cleaning, a human-entered command was required.

Below we describe the search pipeline and the reduction procedures adopted to take the raw images to the point where they are transferred to the central SN database server at Fermilab.

\subsubsection{PHOTO}

The raw data are first processed through the Photo pipeline (Lupton et al. 2001, 2002; Stoughton et al. 2002). The software produces the corrected frames and generates bad-pixel maps, position-dependent point-spread functions (PSFs), and astrometric solutions (Pier et al. 2003) that are used in subsequent processing stages. Our version of Photo does not identify sources in the images or perform photometric measurements (Hogg et al. 2001; Smith et al. 2002; Ivezić et al. 2004; Tucker et al. 2006), as they are not appropriate for efficiently identifying SN candidates, which are usually blended with their host galaxies. As with all standard SDSS data, images from each camera column are written in frames of $2048 \times 1361$ unique pixels or $13.51^{\prime} \times 8.98^{\prime}$ on the sky. The pipeline software was slightly tweaked to run on data taken under poor observing conditions (bright moon, low atmospheric transparency, and poor seeing). In 2005 and 2006, approximately $34 \%$ and $30 \%$, respectively, of the frames were acquired when the Moon was above the horizon. Figure 2 shows the distributions of the $r$-band PSF of frames successfully processed through Photo during the first two seasons.

\subsubsection{Image Subtraction}

To identify new transients in the search data, the images are run through a difference imaging pipeline, which matches the PSF of the search and template frames, adjusts for the difference 
in photometric zeropoint, accurately registers their pixels, and performs the subtraction. The image templates were divided into four separate R.A. ranges so that human scanning and target selection could begin well before the entire night of data was finished processing. The subtracted frames in each filter are searched for positive fluctuations that consist of at least 2 contiguous pixels each above $3.0 \sigma$ of the noise. The software is derived from version 7.1 of the Photpipe software used by the SuperMACHO and ESSENCE collaborations (Smith et al. 2002). Extensive changes have been implemented to operate successfully on processed SDSS data. The modified application is called Framesub.

The pipeline is operated in three modes. (1) The first mode is sdssred, where the input of images is prepared for difference imaging. (2) The second one is sdssdiff, which performs a difference imaging analysis of the search data compared to the template data. To conserve computing time, only the gri frames are differenced and used for object detection. This is the default mode that produces the objects for manual inspection, and subsequently the SN candidates described below. (3) The third mode is sdssforce, in which, after reliable detection of a supernova candidate, we difference all data on the object, including the $u$ and $z$ bands, and perform forced-positional photometry at the location of the candidate. The measurements of magnitudes by sdssdiff and sdssforce are referred to as search and forced photometry. The overall structure of the software and detailed descriptions of each mode are provided in Appendix A.

\subsubsection{Object Detection and Filtering Algorithm}

The individual peaks found by Framesub are filtered through a software called doObjects to remove statistical fluctuations and identify true astronomical sources. First, the single-filter peaks are matched by position to identify sources that are detected in at least two of the three gri filters within $0.8^{\prime \prime}$. These sources are flagged as objects. Significant negative fluctuations are not flagged. The list of objects is compared against the list of known variable sources (the veto catalog) constructed from previous scans of Stripe 82 . Any object that matches the position of a known variable is filtered out. Most of the sources in this catalog are variable stars, active galactic nuclei (AGN), and other persistently varying sources. Approximately 10\% (20\%) of the objects identified by doObjects in 2005 (2006) were associated with sources in the veto catalog.

Given the large area covered by the survey and the overlap of the central R.A. range of Stripe 82 with the ecliptic plane, our detections are overwhelmingly dominated by solar system objects. The challenge is to remove as many of these objects as possible prior to handscanning, without filtering out real SN candidates. Objects with proper motions in excess of $\sim 1^{\prime \prime}$ per minute are easily rejected by requiring that the object is detected in the $r$ and $i$ bands within $0.8^{\prime \prime}$ (the filters are imaged in the order riuzg and the effective time difference between adjacent filters is $71.7 \mathrm{~s}$ ). Objects that move as slowly as $\sim 0.2^{\prime \prime}$ per minute can be identified through motion between the $g$ and $r$ bands. Objects that move at a slower rate are slightly more difficult to identify; when they pass in front of a background galaxy, they can look like perfectly good SN candidates. Approximately 35\% (40\%) of the objects found in 2005 (2006) were tagged as moving objects and removed prior to human evaluation.

New transient sources are entered into a dedicated MySQL database and the cutout images are transferred to Fermilab for visual inspection.

\subsection{Handscanning, Autoscanning, and SN Candidate Selection}

In addition to epochs of SN light-curves, the difference imaging and object detection algorithms described above result in the detection of many background sources of variation, both physical and non-physical. In order to robustly reject background and allow us to focus further analysis on promising $\mathrm{SN}$ candidates, we require that a person visually inspect images of the objects, a process which we refer to as handscanning.

In addition to moving solar system objects, other major sources of contamination include artifacts caused by (1) subtraction of slightly misaligned images, which creates shapes with clusters of positive and negative counts (objects that we call dipoles) in the differenced images, (2) diffraction spikes, and (3) bright saturated stars. Satellite trails that are not properly masked by the software also contribute to the background. A small fraction of the dipoles are high proper-motion stars that have drifted between the epochs of the template and search images. Many of these background objects, however, can be quickly rejected by visual inspection. In Figure 3, we show a gallery of cutout search, template, and differenced images of various types of objects evaluated by scanners-"none," "artifact," "dipole," "variable," "transient," "SN gold," "SN bronze," and "SN other." All images shown are from the $r$ band. In Figure 4 , we show gri images of a moving object correctly flagged by the software. These images show the source moved between the $g$ and $r$ exposures, which are separated by about $5 \mathrm{~min}$.

As a convenient way for people to handscan the detected objects, a Web interface was constructed that queries the database and displays all of the relevant information about the object, including gri cutout images of the search, template, and differenced images, measured magnitudes, the mean sky coordinates and the relative positions measured in each filter, and a list of all previous detections (if any) within $0.8^{\prime \prime}$ of the object under inspection. The scanner evaluates the information and decides to either tag the object as a possible $\mathrm{SN}$ or to reject it as background. If an object is tagged as an $\mathrm{SN}$ and there are no previous detections of objects within $0.8^{\prime \prime}$, it becomes a new SN candidate with a unique SN ID number. A candidate will always remain a candidate unless it is manually vetoed by one of the scanners (which rarely occurs). All of the candidates can be accessed through a public Web site ${ }^{40}$.

During the 2005 observing season, we required that every object that is not rejected by doObjects be handscanned by a person. An average of about 3000-5000 objects were inspected per full night of imaging during the fall 2005 season. Six scanners were on duty on a given night, each scanner responsible for inspecting all objects from one of the six camera columns, or $500-800$ objects. Based on our experience in 2005, we made a number of changes to the handscanning procedure for the 2006 season described below.

\subsubsection{Autoscanner}

To reduce the number of objects to be handscanned, prior to the 2006 season we implemented a new software filter, called the autoscanner. The software performs two primary tasks: (1) it identifies all objects detected in more than one epoch as well as bright ( $g$ or $r<21 \mathrm{mag}$ ) objects detected for the first time, and (2) it uses statistical classification techniques to identify and filter out first-epoch background non-SN objects.

The reasons for performing (1) are as follows. First, the selection of objects detected in more than one epoch provides a

${ }_{40}$ http://sdssdp47.fnal.gov/sdsssn/candidates/candTable.php. 

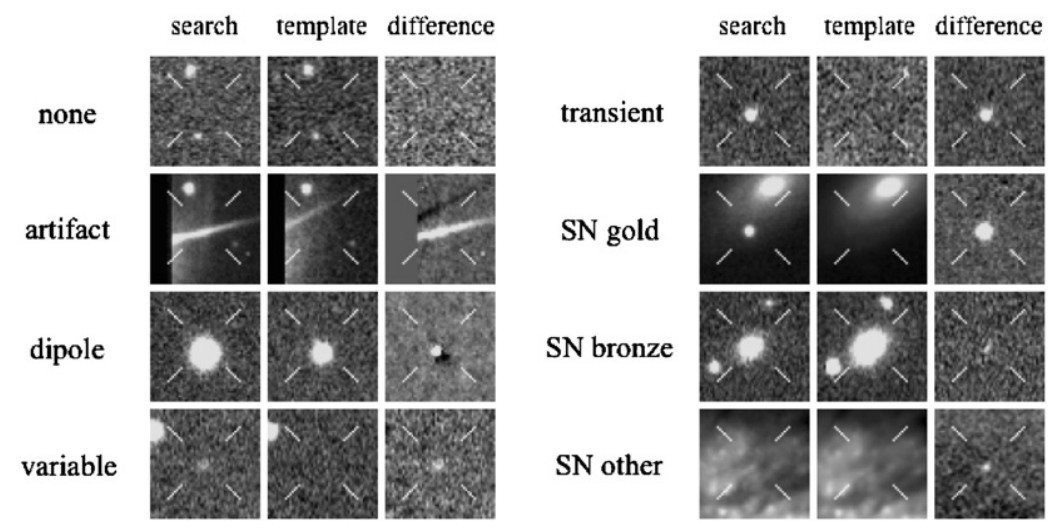

Figure 3. A gallery of various types of objects that a scanner evaluates. The panels show $r$-band search (left columns in each panel), template (central columns), and differenced (right columns) images of objects classified by scanners as "none," "artifact," "dipole," "variable," "transient," "SN gold," "SN bronze," and "SN other." Objects classified as "none" are typically detections near the threshold, and are often not visible to the human eye. Most "artifacts" are caused by different orientations of diffraction spikes in the search and template images. A "dipole" results from imperfect image registration as well as high proper-motion stars. A "variable" is an object associated with a persistent source and usually exhibits random temporal variability. These sources are newly variable sources that are not part of the veto catalog. A "transient" object is usually seen for the first time and not associated with a galaxy. The source can be a true SN with an undetected host galaxy or a slow-moving asteroid. An "SN gold" is a good SN candidate that is well separated from the host. An "SN bronze" is a possible SN that lies close to the core of the galaxy and can, therefore, also be a variable active galactic nucleus. An "SN other" is also a possible SN, but is most likely a different type of variable source-in the case shown above, the source is a variable star in NGC1068 (M77). There is another category called "SN silver" (not shown), which is used for objects that are similar to a "transient", but detected in more than a single epoch (e.g., an SN with an undetected host galaxy). The distinction between gold/silver/bronze/other SN candidates is not important; all objects tagged as an "SN" become an SN candidate. Each cutout image is 20 " on the side.

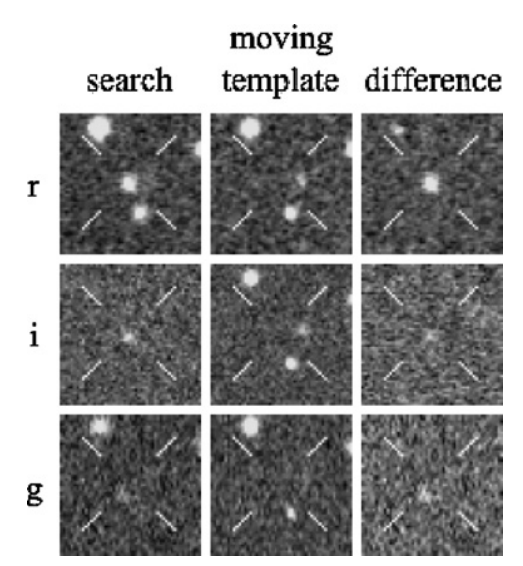

Figure 4. An example of a moving object that was correctly flagged by the autoscanner. As in Figure 3, the search, template, and differenced images are shown in the left, middle, and right columns, respectively. In addition to the $r$-band images (top row), we also display the $g$-band (middle row) and $i$-band (bottom row) images to show the relative position of the source in each filter. Note that the faint source in the $g$-band differenced image appears slightly below and to the left of the center of the image. The images are acquired in the order $r i(u z) g$ with a $71.7 \mathrm{~s}$ delay between each filter.

very robust way of eliminating moving objects, one of the major contaminants of the 2005 SN candidates. Second, the selection of bright first-epoch objects enables us to discover nearby SNe; we can thereby obtain spectroscopic observations well before maximum light and provide rapid alerts to the SN community. In addition, bright, single-epoch candidates provide a back-up list of spectroscopic targets for nights when the spectroscopy queue is not filled by promising multi-epoch candidates. Although most of our targets were spectroscopically observed after two or more epochs of photometry, in a few instances, we did obtain spectroscopic observations of SN candidates based on a single epoch of detection. In the selection of these objects, the autoscanner also identifies objects associated with either a known variable in the veto catalog or a known $\mathrm{SN}$ candidate. In the former case, the object is filtered out. In the latter case, since there is no need for a person to scan the object, it is instead flagged by the autoscanner as a known SN candidate, stored in the database, and used in the light curve analysis (see Section 3.1).

For (2), the autoscanner attempts to identify and filter out bright first-epoch objects that belong to one of three classes of background: unmasked diffraction spikes, artifacts of imperfect image registration (dipoles), and moving objects. The software treats the set of objects, along with their evaluations by a person, from the 2005 search as a training set, and compares the observed quantities for newly detected objects against this set. The method used to classify an object is the histogram method of probability density function (PDF) estimation. In this method the observable quantities, or attributes, of an object form a multidimensional space, and a classification decision is made by considering the number count of objects, in the training set, from each class in a bin centered at the point describing the object we wish to classify. This method has the advantages of being nonparametric, allowing the decision boundaries in the observable space to be arbitrarily complicated, and of explicitly retaining the correlations between observables. A caveat of using the histogram method of PDF estimation is that one must have a sufficient number of objects in the training set to sample the PDF well in all regions of interest. With $\approx 90,000$ objects in the training set, this is not a significant limitation for the SDSS-II SN survey. A technical discussion of the algorithm is presented in Appendix B.

During the 2006 observing season, the autoscanner was used to reject moving objects from among the set of bright firstepoch objects that were to be handscanned. For such bright objects, only $\approx 0.7 \%$ of them were incorrectly tagged as moving objects by the autoscanner. In contrast to the 2005 observing season, we chose not to handscan epochs of known candidates or objects that showed variation in more than one observing season, and the autoscanner was used to reject such objects. Although some objects were classified as artifacts or dipoles, we chose not to reject these from the handscanning as they are a small fraction of the background in comparison to moving 
Table 4

Reduction of 2006 Handscanning by Autoscanner

\begin{tabular}{|c|c|}
\hline & Number of objects \\
\hline Total to be scanned ${ }^{\mathrm{a}}$ & 145,428 \\
\hline First epoch & 118,584 \\
\hline Bright $^{\mathrm{b}}$ & 21,882 \\
\hline Scanned $^{\mathrm{c}}$ & 8,373 \\
\hline Second epoch & 26,844 \\
\hline Scanned & 6,007 \\
\hline Total scanned ${ }^{\mathrm{d}}$ & 14,430 \\
\hline
\end{tabular}

Notes. ${ }^{\text {a }}$ The number of objects that humans would have scanned in 2006 without the autoscanner.

b Objects that were detected for the first time and brighter than $21 \mathrm{mag}$ in $g$ or $r$.

c Actual number of "bright," first-epoch objects that were scanned.

d Total number of objects scanned in 2006. This number differs slightly from the sum of the scanned first- and second-epoch objects because some scanners on a few occasions chose to scan first-epochs objects as faint as $21.5 \mathrm{mag}$ in $g$ or $r$.

objects, and the relatively small reduction in handscanning that would be accomplished was determined to be outweighed by the risk of missing an early epoch of a nearby SN.

This new handscanning strategy and the autoscanner enabled a reduction in the number of objects scanned by more than an order of magnitude between 2005 and 2006, with no reduction in the quality or quantity of confirmed SNe. While scanning a single camera column in 2005 typically took $2-3 \mathrm{~h}$ per person for a full night of data, in 2006 a scanner could cover two columns in only 10-20 min. The reduction of the 2006 handscanning by the autoscanner is summarized in Table 4.

A comparison of the objects classified by the autoscanner as artifacts to their human classification shows that the autoscanner is extremely efficient at recognizing artifacts, and that our concern about potentially rejecting nearby $\mathrm{SNe}$ was overly cautious. During the 2006 observing campaign, 3753 objects had been classified by the autoscanner as artifacts. Human scanners classified 2668 of these objects as artifacts and 3710 of them as some type of non-SN background. Of the remaining 43 objects classified by people as SN candidates, 41 were background (non-SN) erroneously classified by the scanner, one was a bright nova outburst, and one was the first epoch of an SN that was later (after the second-epoch detection) classified as an SN. Therefore, in cases where they disagree on classification of artifacts, the autoscanner appears to be more reliable than the human scanners.

\section{SPECTROSCOPIC TARGET SELECTION AND FOLLOW-UP OBSERVATIONS}

Spectroscopic follow-up is an essential component of any current supernova survey, for both confirming the type of the SN candidate and measuring the cosmological redshift (preferably from host galaxy emission/absorption lines). For the SDSS-II SN survey, the amount of scheduled spectroscopic telescope time is larger than the amount of imaging time on the SDSS $2.5 \mathrm{~m}$ telescope by a large factor (see also Frieman et al. 2007). The telescopes used for spectroscopy in 2005 and 2006 included the $9.2 \mathrm{~m} \mathrm{HET}, 3.6 \mathrm{~m}$ NTT, $3.5 \mathrm{~m}$ ARC, $8.2 \mathrm{~m}$ Subaru,
2.4 m MDM Hiltner, 4.2 m WHT, 4 m KPNO Mayall, 10 m Keck, $2.5 \mathrm{~m}$ NOT, and the $11 \mathrm{~m}$ SALT.

On any given night during the fall season, there could be several telescopes that are simultaneously scheduled and observing a set of our SN candidates. This requires a significant amount of coordination between the various observers at each of the telescopes to avoid observing the same candidate. A single person (the first author of this paper) was responsible for coordinating all follow-up imaging and spectroscopic observations in 2005 and 2006. Telescopes separated by several time zones are relatively easy to coordinate, but those that have similar longitudes (e.g., MDM, ARC, KPNO) require real-time communication. In general, the brighter targets are parsed to telescopes with smaller aperture, but the magnitude limits must be adjusted depending on the observing conditions at each site. We have made every effort to avoid taking duplicate spectra, but on occasion the same object was observed nearly simultaneously on two telescopes.

A good night of imaging of half of Stripe 82 typically yields 100-300 objects that are tagged as new SN candidates, which are publicly accessible through the $\mathrm{Web}^{41}$ immediately after they are entered into the database. This number significantly exceeds the number of targets we can observe spectroscopically, and choices must be made. The following subsections describe the further prioritization of SN targets for spectroscopic observations. The algorithm and strategy depends on the amount of resources available, which is something that evolved quite substantially between the 2005 and 2006 search seasons.

\subsection{SN Photometric Typing}

After each night of imaging on the SDSS $2.5 \mathrm{~m}$ telescope, the gri light curves of all active candidates are compared against a library of light curve templates of different SN types. The purpose of this procedure is twofold: (1) to quickly identify the best-matching template and provide estimates of the redshift, extinction, the approximate date of maximum light, and current apparent magnitudes, and (2) to compute other quantities that are useful for prioritizing follow-up spectroscopy, such as the amount of host galaxy contamination. The process is essential since the number of active SN candidates (a good fraction of which are not $\mathrm{SNe}$ ) at any given time exceeds the number that can be observed spectroscopically by a large factor. A reliable system is necessary to make efficient use of the valuable spectroscopic resources. Several techniques and algorithms for SN photometric identification have been introduced previously by Poznanski et al. (2002, 2006), Johnson \& Crotts (2006), Riess et al. (2004b), Sullivan et al. (2006), Kunz et al. (2007), and Kuznetsova \& Connolly (2007). The method adopted here is most similar to that used by the SNLS spectroscopic followup described in Sullivan et al. (2006). Below, we describe the details of the algorithm.

The measured light curves are compared against a library of light curve templates, which are grouped into three types$\mathrm{SN}$ Ia, SN Ib/c, and SN II-and are generated from multiepoch spectra constructed and compiled by P. Nugent ${ }^{42}$ and also from the SUSPECT database ${ }^{43}$. Specifically, for the type Ia, we use Nugent's Branch-normal, 1991T-like, and 1991bg-like templates. The Branch-normal spectra are used for computing synthetic light curves of $\mathrm{SNe}$ Ia with a range of luminosities parameterized by the decline rate. The model is described

\footnotetext{
41 http://sdssdp47.fnal.gov/sdsssn/candidates/candTable.php.

$42 \mathrm{http}: / /$ supernova.lbl.gov/nugent/nugent_templates.html.

$43 \mathrm{http} / / /$ bruford.nhn.ou.edu/suspect/index $1 . \mathrm{html}$.
} 
in Appendix C. The set of templates used for the type Ib/c are Nugent's normal and hypernova Ib/c spectra, as well as spectra and light curves of SN1999ex and SN2002ap from the SUSPECT database. Similarly, we use Nugent's II-P, IIL, and IIn spectra, and SUSPECT's 1993J (IIb), 1998S (IIn), and SN1999em (II-P) to generate a set of type II light curves.

The light curves in the observed ugriz filters are calculated on a grid of four parameters $\left(z, A_{V}, T_{\max },\left[\Delta m_{15}(B)\right.\right.$, template $\mathrm{SN}]$ ), where $z$ is the redshift, $A_{V}$ is the host galaxy extinction in the $V$ band and assumes $R_{V}=3.1$, and $T_{\max }$ is the time of rest-frame $B$-band maximum light. The last parameter refers to either the decline rate parameter for the Branch-normal Ia models $\left(\Delta m_{15}(B)\right)$, or the particular SN template for the peculiar SNe Ia (1991T-like and 1991bg-like) and the core-collapse models. We do not attempt to fit or correct for the Milky Way extinction. In this procedure, we assume a cosmology to convert the redshift to a luminosity distance, similar to the method adopted by the SNLS described in Sullivan et al. (2006). The adopted cosmological parameters are $\Omega_{m}=0.3$ and $\Omega_{\Lambda}=$ 0.7 . For the SN Ia templates, we also assume a fiducial peak $B$-band absolute magnitude of $M_{B}=-19.0+5 \log \left(H_{0} / 70\right)$ mag, where $H_{0}$ is the Hubble constant in units of $\mathrm{km} \mathrm{s}^{-1} \mathrm{Mpc}^{-1}$, for a standard $\Delta m_{15}(B)=1.1 \mathrm{SN}$ Ia. As shown by Sullivan et al. (2006), a particular set of assumed cosmological parameters in the computation of the model light curves does not significantly bias the population of targets for spectroscopic observations. At low redshift $(z \lesssim 0.2)$, the luminosity distances are not sensitive to our choice of $\Omega_{m}$ and $\Omega_{\Lambda}$. Above $z \sim 0.2$, the statistical uncertainties in the fluxes and the combination of varying $z$ and $A_{V}$ can compensate for differences in luminosity distance that might result from a different set of cosmological parameters. The assumption, however, could systematically bias the estimated photometric redshifts, but that is not a concern for the purposes of target selection.

The templates are grouped into three $\mathrm{SN}$ types- $-\mathrm{Ia}$, Ibc, and II-and the fitter records the best-fit parameters and the minimum value of the $\chi^{2}$ statistic on the four-dimensional parameter grid within each of the three types. In addition to identifying the SN type with the lowest $\chi^{2}$ value, which we refer to as "type-best," we examine the relative values of $\chi^{2}$ and determine whether the SN candidate should be considered as "typed" according to one or both of the following two criteria. If we denote the value of $\chi^{2}$ of the best-matching type by $\chi_{\min }^{2}$, the next best type as $\chi_{1}^{2}$, and the worst one as $\chi_{2}^{2}$ (i.e., $\chi_{\min }^{2}<\chi_{1}^{2}<\chi_{2}^{2}$ ), the "A" criterion is satisfied if $\chi_{\min }^{2}<\chi_{1}^{2}-\chi_{\min }^{2}$. If "type-best" is a Ia and satisfies the above criterion, the candidate is said to have a "type-A of Ia". The "B" criterion is satisfied if $\chi_{\min }^{2}<N\left(\chi_{\min }^{2}+\chi_{1}^{2}+\chi_{2}^{2}\right) / 3$ with $N=$ 0.5 . Similarly, if the best-fit type is an SN Ia and satisfies the above $\chi^{2}$ criterion, the candidate is said to have a "type-B of Ia". The value of 0.5 adopted for $N$ works well for $\mathrm{SN}$ candidates with a low $\mathrm{S} / \mathrm{N}$, and was empirically determined from a sample of spectroscopically confirmed SNe from our 2004 engineering run (Sako et al. 2005). Also computed are the estimated current $g$ and $r$ magnitudes from the model light curves. We also search for the nearest galaxy within $10^{\prime \prime}$ from the SN position in the SDSS galaxy catalog and refit and retype the light curves using the best estimate of its redshift as a prior. We adopt galaxy photometric redshifts from Oyaizu et al. (2007) and spectroscopic redshifts from the SDSS DR5 (Adelman-McCarthy et al. 2007).

We note that the absolute $\chi^{2}$ values for the model fits do not appear to be very meaningful. The models have not been calibrated against real data and there are no errors associated

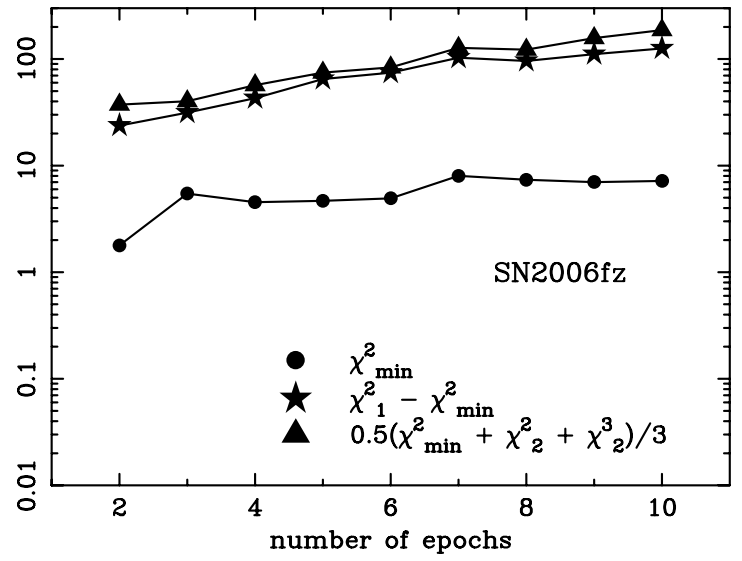

Figure 5. Fit results for SN2006fz (SN Ia at $z=0.105$; internal candidate ID 13135) as functions of the number of epochs in the light curve. The bottom curve (labeled with filled circles) shows the minimum $\chi^{2}$ values, which at every epoch correspond to the Ia model. The top two curves labeled with filled stars and triangles show values relevant for the "A" and "B" typing criteria, respectively. For this particular SN, $\chi_{\min }^{2}$ is always below these two curves, which implies that this $\mathrm{SN}$ is a type Ia at high confidence.

with the light curves (see, e.g., Appendix C). The current implementation also does not attempt to reject outlier points due to poor zeropointing and imperfect image registration, which are common features of the search photometry. The best-fit $\chi^{2}$ value for the SN Ia model of a true SN Ia may be large compared to the number of degrees of freedom, but the confidence can be high if that value is well below the values of the other SN types. The relative values of $\chi^{2}$ are, therefore, important and useful discriminators. In Figure 5, we show the values of $\chi_{\min }^{2}$, $\chi_{1}^{2}<\chi_{2}^{2}$, and $0.5\left(\chi_{\min }^{2}+\chi_{1}^{2}+\chi_{2}^{2}\right) / 3$ as functions of the number of epochs for SN 2006fz, a type Ia at $z=0.105$, with a flat galaxy photometric redshift prior. For this candidate, the model with the minimum $\chi^{2}$ corresponds to the SN Ia model, and both the "A" and "B" criteria are satisfied at all epochs. Also shown in Figure 6 are the light curve fits to SN 2006fz using the first $2,4,6$, and 8 epochs of search photometry.

The overall performance of the photometric typing software is demonstrated in Figure 7, where we plot the fraction of the spectroscopically confirmed $\mathrm{SNe}$ Ia, whose best-fit model is that of an SN Ia as a function of the number of the light curve epochs. The figure also shows the fraction of SN Ia that satisfy one of the "A" or "B" criteria with or without a nearest-neighbor host galaxy redshift prior. Note that the fractions increase between 2 to $\sim 4$ epochs, but the improvement beyond $\sim 5$ epochs is marginal. The fraction with the best type equal to a Ia does not reach unity because the spectroscopic sample includes (1) peculiar SNe Ia, (2) candidates with poorly subtracted images and photometry epochs, (3) SNe Ia discovered well after maximum light, and (4) $\mathrm{SNe}$ with low-S/N light curves whose best-fit type drifts with the number of epochs. Candidates that fall into the first three categories were targeted for spectroscopy because they were nearby and bright enough to be observed on $3 \mathrm{~m}$ class telescopes, and those candidates were observed even if their best-fit photometric type was not an SN Ia (see Section 3.3). The candidates in the fourth category were observed because they were at one time typed as an SN Ia. We also show in Figure 8 the time of $B$-band maximum light estimated from 2-4 light curve epochs in comparison with the "final" estimate of the peak using the full light curves. Again, the reliability improves substantially between 2 and $\sim 4$ epochs. 

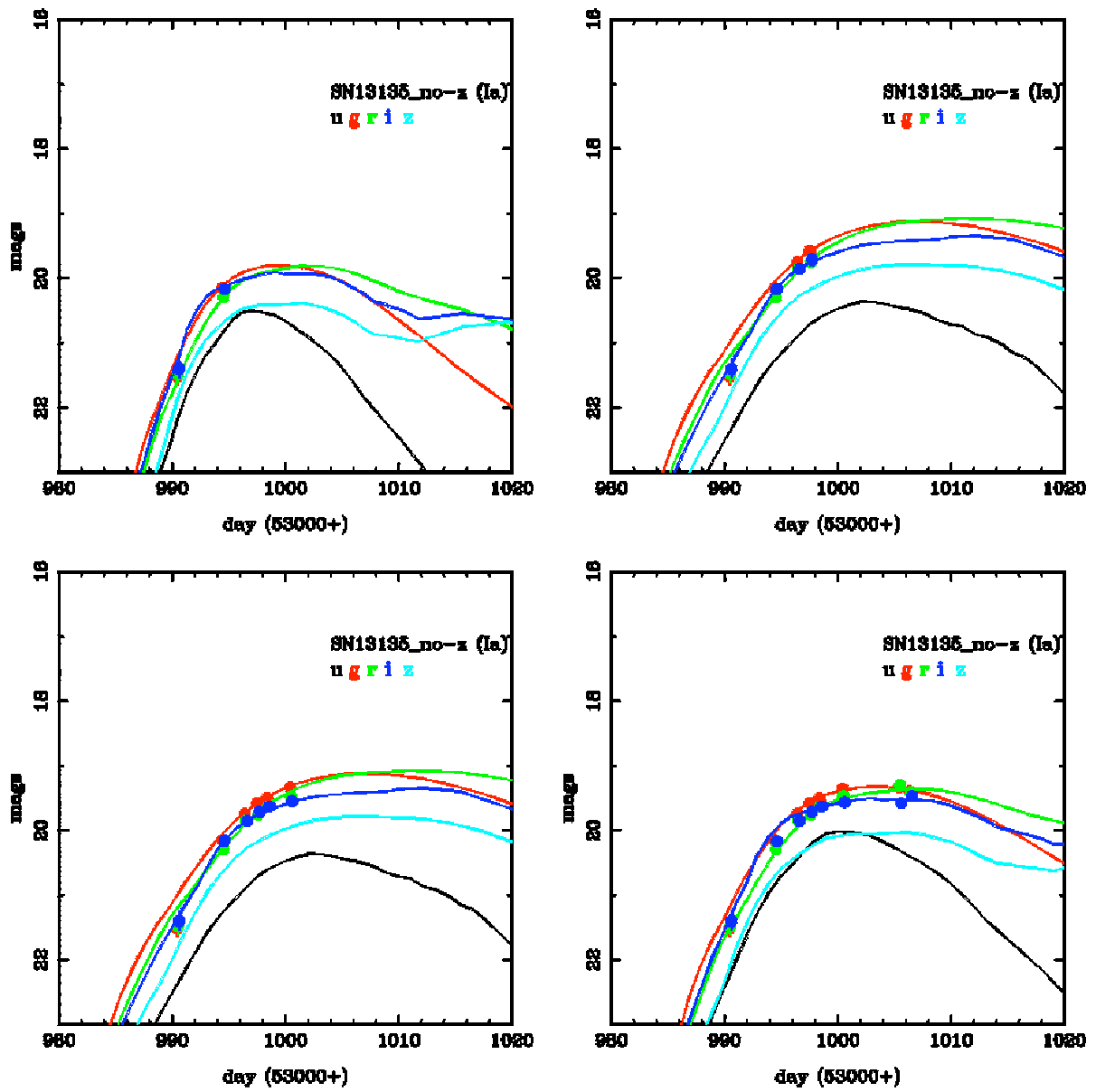

Figure 6. Light curves of SN2006fz (the same SN as shown in Figure 5) and fits to the best-fit SN Ia models after 2, 4, 6, and 8 epochs of detections without a galaxy photometric redshift prior. Search photometry from gri are shown. Model light curves in $u$ and $z$ are shown as well.

(A color version of this figure is available in the online journal)

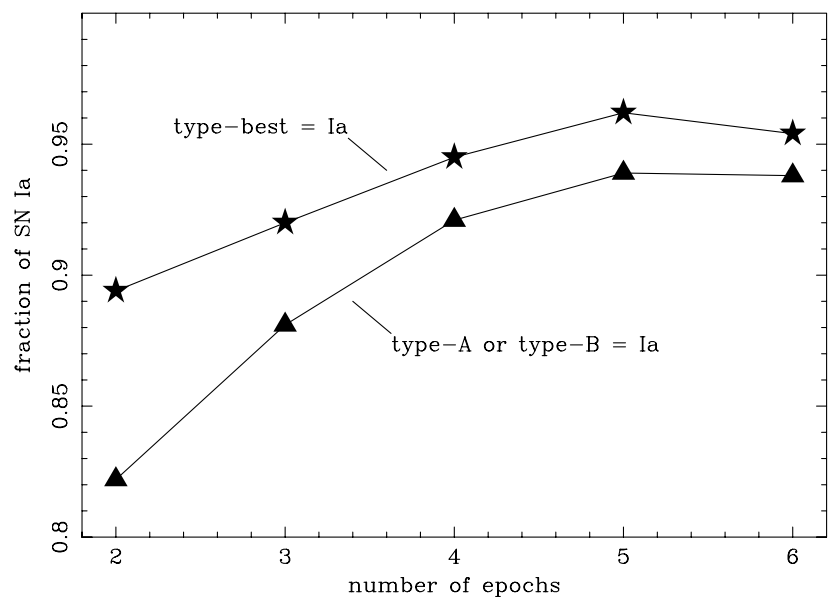

Figure 7. The fraction of spectroscopically confirmed SNe Ia whose best-fit model is that of an SN Ia as a function of the number epochs (top curve with stars). Also shown is the fraction of SNe Ia, which satisfy one of the "A" or "B" criteria with or without a galaxy redshift prior (bottom curve with triangles). A large fraction of the sources $(>80 \%)$ are typed as SN Ia after only 2 epochs of imaging.
Studies of the software and handscanning efficiencies as functions of the object brightness with the fakes (see Section A.2) show that the SN candidate identification and spectroscopic follow-up observations are essentially complete out to $z \sim 0.12$ (Dilday et al. 2007). The one exception to this is our bias against highly-extincted $\mathrm{SNe}$, since the grid of light curve models did not extend beyond $A_{V}=1.0$ in 2005 and $A_{V}=3.0$ in 2006. A post-season analysis of the 2005 candidates with an extended $A_{V}$ range recovered two additional photometric SN Ia candidates with large extinction in this redshift range. There is no evidence for an additional population of low- $z$ SNe Ia that were missed due to the models adopted.

The light curve fits generally run on the gri search photometry, which is usually accurate to within $\sim 0.1 \mathrm{mag}$. Whenever the computer cluster at APO is idle, we run the pipeline in sdssforce mode (see, Section 2.2.2) to produce $u$ and $z$-band differenced images from all runs available on the mountain for each of the SN candidates. This typically includes frames from several pre-discovery epochs. The $u$-band photometry is sometimes useful for distinguishing $\mathrm{SNe}$ Ia from core-collapse $\mathrm{SNe}$ that are generally bluer $(u-g \lesssim 0.5)$ during the early phases after explosion. 


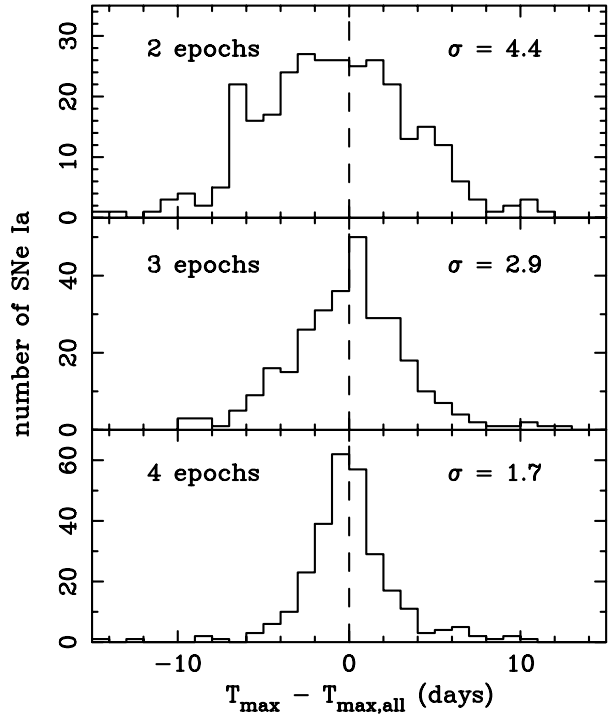

Figure 8. Distribution of the difference between the time of $B$-band maximum estimated using the full light curves and that using the first 2 (top), 3 (middle), and 4 (bottom) epochs of the spectroscopically confirmed SNe Ia. Values are taken from fits with a flat redshift prior. The distributions are fit with a single Gaussian, and the $\sigma$ values are listed in each panel. The width of the distribution stays approximately constant above 4 epochs. The vertical dashed lines represent the $x$-axis origin, not the centroid of the distributions.

\subsection{Observability Index}

After the SN photometric typing process, the software computes three quantities that help prioritize the target list for spectroscopic observations. These quantities are referred to as "weights" and they are constructed specifically to identify candidates that are (1) near or before peak brightness, (2) less contaminated by galaxy light, and (3) not heavily extincted by intervening dust. The product of the three weights defines the "observability index"; candidates with a higher observability index are generally assigned higher priority.

For (1), we define the "time weight" as,

$$
W_{T}= \begin{cases}e^{|t|\left\{\Delta m_{15}(B) /[10(1+z)]\right\}} & \text { if } t<0 \text { days, } \\ e^{-t\left\{\Delta m_{15}(B) /[20(1+z)]\right\}} & \text { if } t \geqslant 0 \text { days }\end{cases}
$$

where $t$ is the current estimate of the $\mathrm{SN}$ epoch in days relative to $B$-band maximum brightness. Note that $W_{C}$ is large when the $\mathrm{SN}$ is young, as $\mathrm{SN}$ are most easily classified near maximum light (Filippenko 1997), and decays exponentially with a characteristic time scale of 20 days. The decline-rate parameter $\Delta m_{15}(B)$ and redshift $z$ are also adopted from the best-fit values obtained from the light curve fits. If the best-fit type is a core-collapse $\mathrm{SN}, \Delta m_{15}(B)$ is set to unity.

The second weight computed by the software is the "contamination weight" defined as

$$
W_{C}=e^{-2 / \theta} \times\left(\frac{F_{\mathrm{SN}, r}}{F_{\mathrm{Gal}, r}}\right)^{1 / 2},
$$

where $\theta$ is the distance in arcseconds between the $\mathrm{SN}$ and the centroid of the nearest-neighbor host galaxy. The quantity $F_{\mathrm{SN}, r}$ is proportional to the current $r$-band flux of the $\mathrm{SN}$, again, as estimated from the best-fit light curve model,

$$
F_{\mathrm{SN}, r}=10^{-0.4 r_{\mathrm{SN}}}
$$

and $F_{\mathrm{Gal}, r}$ is the estimated local galaxy $r$-band flux at the position of the $\mathrm{SN}$ defined to be

$$
F_{\mathrm{Gal}, r}=10^{-0.4\left(r_{\mathrm{Gal}}+d / d_{\mathrm{eff}}\right)},
$$

where $d$ is the angular separation between the $\mathrm{SN}$ and the centroid of the nearest-neighbor source and $d_{\mathrm{eff}}$ is the effective radius of that source along the position angle of the SN. This quantity is computed according to

$$
d_{\mathrm{eff}}=\frac{i \operatorname{soA}^{2}\left(1-e^{2}\right)}{1-e^{2} \cos ^{2} \phi} .
$$

Here, $\phi$ is the position angle of the $\mathrm{SN}$ measured from the position angle of the host galaxy's semimajor axis (isoPhi) with ellipticity given by

$$
e^{2}=1-\left(\frac{\text { isoB }}{\text { isoA }}\right)^{2}
$$

The isophotal galaxy parameters (isoA, isoB, and isoPhi), which are used to measure the galaxy's ellipticity of the $25 \mathrm{mag} \mathrm{arcsec}^{-1}$ isophote, are adopted from the SDSS DR4 catalog 44 . Clearly, candidates with larger values of $W_{C}$ suffer less contamination from the host galaxy light.

The third and final weight is the "dust weight," which estimates the amount of dust extinction from both host galaxy morphology and color, and is defined to be

$$
W_{D}=e^{-d_{\text {eff }} / d} \times\left(1+3 f_{\mathrm{deV}}+5 c_{\text {ellip }}\right) .
$$

The quantity $f_{\mathrm{deV}}$ is the fractional probability of the source being well represented by a de Vaucouleurs profile and is again computed from values in the SDSS catalog, i.e.,

$$
f_{\mathrm{deV}}=\frac{\mathrm{deV} \_\mathrm{L}}{\mathrm{deV} \_\mathrm{L}+\exp \_\mathrm{L}+\operatorname{star} \_\mathrm{L}},
$$

where deV_L, exp_L, star_L are the null hypothesis probabilities of the object being well represented by a de Vaucouleurs profile, an exponential function, and a stellar profile (PSF), respectively. The last quantity in parentheses of Equation (7) characterizes the color of the galaxy, and is defined to be

$$
c_{\text {ellip }}= \begin{cases}\left(r_{\mathrm{Gal}}-i_{\mathrm{Gal}}-0.4\right) / 0.2 & \text { if }\left(r_{\mathrm{Gal}}-i_{\mathrm{Gal}}\right)>0.4 \\ 0 & \text { and }\left(g_{\mathrm{Gal}}-r_{\mathrm{Gal}}\right)>0.9, \\ & \text { otherwise }\end{cases}
$$

where $g_{\mathrm{Gal}}, r_{\mathrm{Gal}}, i_{\mathrm{Gal}}$ are the host galaxy $g, r$, and $i$-band model magnitudes. This quantity is non-zero only for red elliptical galaxy candidates, which typically populate the color-color space bounded by $(r-i)>0.4$ and $(g-r)>0.9$. Note that the light curves also provide an independent (and usually more reliable) estimate of the amount of dust extinction.

The final observability index is defined as the product of the three weights,

$$
\text { observability index }=W_{C} \times W_{T} \times W_{D} .
$$

In practice, the observability index is rarely used for the bright $(r \lesssim 20.5$ mag) low- $z$ SN candidates, since there are enough spectroscopic resources to observe nearly all of those targets. At high $z$, however, the number of SN Ia candidates exceeds

\footnotetext{
${ }^{44}$ http://www.sdss.org/dr4/algorithms/classify.html.
} 
the number we can observe given our limited resources on large-aperture telescopes. In order to select the best targets from a large number of candidates that satisfy the light curve selection criteria, the sources are ranked based on the value of the observability index. Manual screening and selection of the sources by the spectroscopic coordinator is still required. We describe our strategy in detail below.

\subsection{Spectroscopic Follow-up Strategy}

Spectra are obtained by the various spectroscopic teams, who independently apply for observing time during each of the search seasons. The teams agree to confirm types and obtain redshifts of SN Ia candidates at the highest priority, but each team generally has one or more of their own SN projects that they pursue in parallel. Some of these include (1) multi-epoch spectroscopy of peculiar $\mathrm{SNe}$, (2) $\mathrm{SNe}$ in underluminous host galaxies, (3) $\mathrm{SN} \mathrm{Ib/c} \mathrm{and} \mathrm{hypernova} \mathrm{candidates,} \mathrm{(4)} \mathrm{detailed} \mathrm{spectroscopic}$ properties of SN Ia hosts, and (5) multi-epoch studies of line features and their diversity in SNe Ia. Whenever possible, we also try to obtain spectra of other variable objects if a scanner has classified them as "interesting." An example of this is a dwarf nova discovered in Aquarius (Prieto et al. 2006).

In 2005, the primary telescopes used for observing the lowredshift candidates were the ARC $3.5 \mathrm{~m}$, WHT $4.2 \mathrm{~m}$, and the MDM $2.4 \mathrm{~m}$ telescopes. The high- $z$ candidates were observed with either the HET or Subaru. The resources were sufficient to obtain spectra of most $z \lesssim 0.15 \mathrm{SN}$ Ia candidates as well as core-collapse $\mathrm{SNe}$ at $z \lesssim 0.06$, but only a fraction of the $z \gtrsim 0.15$ candidates were observed.

During the fall 2006 campaign, the various spectroscopic teams were awarded sufficient $3 \mathrm{~m}$ class telescope time to obtain a spectrum of essentially all SN candidates with $r \lesssim 20.5$ mag (an average SN Ia at $z \lesssim 0.15$ and core-collapse $\mathrm{SN}$ at $z \lesssim 0.06$ near maximum light) and obtain multi-epoch spectra of a large sample of nearby $(z \lesssim 0.1)$ SNe Ia.

We generally require at least two epochs of detection before we target the candidate for spectroscopic observation, to guarantee that the source is not an asteroid. This also enables a better estimate of the epoch of peak brightness of the SN. Since the primary goal of our survey is to obtain well-sampled light curves of type Ia SNe, SN Ia candidates that are discovered early are generally given higher priority for spectroscopy. This is especially true for the very low $z$ candidates $(z \lesssim 0.1)$ to enable rapid confirmation and dissemination to the public.

In practice, we initially select SN Ia candidates that satisfy at least one of the "A" or "B" criteria either with or without a galaxy photometric redshift prior. There are times, however, during stretches of poor weather at APO and/or when several $3 \mathrm{~m}$ class spectroscopic telescopes are online, when there are not enough new and bright SN Ia candidates to observe. This was not an issue during most of the 2005 season, but it occurred frequently in 2006. In such cases, we loosen the selection criteria and inspect all currently-bright sources irrespective of their bestfit $\mathrm{SN}$ type; there is only a small number $(\sim 30-50)$ of sources with $r \lesssim 20.5 \mathrm{mag}$ at any given time. When necessary, we also target candidates that have only a single epoch of detection. Extremely red single-epoch targets that are not associated with a host galaxy are generally avoided (to minimize the chance of observing an asteroid).

On the other hand, there is never a shortage of higher- $z$ SN Ia targets at $z \gtrsim 0.2$, so the "A" or "B" criterion with or without a galaxy photometric redshift prior is always used in the pre-selection process. At these redshifts, we generally avoid candidates that are located within $\sim 0.5^{\prime \prime}$ of the centroid of a host galaxy, since they have a relatively higher chance of being an active galactic nucleus. Candidates at $z \sim 0.3$ that are well separated from the host and suffer a relatively low level of extinction (as estimated from the multi-band light curve fits) are high-priority (priority 0 and 1) targets for HET, which is a queuescheduled telescope (Shetrone et al. 2007). Lower- $z$ candidates $(z \sim 0.2)$ are assigned lower HET priority (mostly priority 2 , but some at priority 3 ). Targets are generally kept active in the HET queue until they are $\sim 20$ days past maximum light as estimated from the light curve fits. The Subaru telescope has focused on SN Ia candidates in a similar redshift range, but, to take advantage of its superb image quality, the Subaru telescope has generally been assigned targets that suffer a relatively large amount of host contamination (i.e., a low value of $W_{C}$; see Section 3.2).

The spectra are often analyzed in real time by the observers on site, and feedback is given to the spectroscopic coordinator. Depending on the observing conditions, the target list is adjusted in real time. At the end of the night, the observers provide a preliminary spectroscopic type of each of the candidates observed. The type and the preliminary redshift is entered immediately into the database, and the results are disseminated to the community through the Central Bureau Electronic Telegrams. All nearby $\mathrm{SNe}(z \lesssim 0.08)$, however, are also announced to a list of subscribers via email usually within hours from confirmation, allowing rapid complementary follow-up on other telescopes. Information on all spectroscopically confirmed $\mathrm{SNe}$ is placed on a public Web page ${ }^{45}$ as soon as the $\mathrm{SN}$ type has been entered into the database. Lists of all spectroscopically confirmed SNe from 2005 and 2006 are presented in Tables 5 and 6, respectively.

\section{IMAGING FOLLOW-UP}

Throughout the 2005 search, we used some of our ARC $3.5 \mathrm{~m}$ and MDM $2.4 \mathrm{~m}$ time, as well as the imager on the NMSU $1 \mathrm{~m}$ telescope, University of Hawaii 88 inch telescope, the $1.8 \mathrm{~m}$ Vatican Advanced Technology Telescope (VATT), and the $3.5 \mathrm{~m}$ WIYN telescope to (1) augment the light curve points of spectroscopically confirmed SNe Ia during periods of poor weather conditions at APO and (2) obtain late-time photometry of SNe that had faded below the SDSS detection limit. Some imaging observations were performed by the VATT, the WIYN, and the $1.5 \mathrm{~m}$ telescope at Maidanak Observatory during the 2006 season. Additional imaging data were obtained on the ARC $3.5 \mathrm{~m}$ during November of 2006 when the Dual Imaging Spectrograph was not functional. This effort of follow-up imaging was not carried over for the MDM and for ARC during September and October, since we concluded that spectroscopy is the more valuable use of our resources. In December of both 2005 and 2006, however, imaging observations were performed to get light curves of SNe discovered in November past maximum light. Deep multi-band imaging of several host galaxies of SNe Ia were also obtained by the $2.5 \mathrm{~m}$ Isaac Newton Telescope.

A few high-profile SDSS-II SN targets were also observed extensively by several follow-up programs: optical imaging and spectroscopy by the CfA SN group, optical and infrared imaging and optical spectroscopy by the CSP, and optical spectroscopy by the SNFactory.

\footnotetext{
${ }^{45}$ http://sdssdp47.fnal.gov/sdsssn/snlist_confirmed.php.
} 
Table 5

List of Spectroscopically Confirmed Supernovae in 2005

\begin{tabular}{|c|c|c|c|c|c|c|c|}
\hline \multirow{2}{*}{$\begin{array}{l}\text { Candidate } \\
\text { ID }^{\mathrm{a}}\end{array}$} & \multirow{2}{*}{$\begin{array}{c}\text { IAU } \\
\text { Name }\end{array}$} & \multirow[b]{2}{*}{$z^{\mathrm{b}}$} & R.A. ${ }^{c}$ & Decl. & \multirow{2}{*}{$\begin{array}{c}\text { Discovery } \\
\text { date }\end{array}$} & \multirow[b]{2}{*}{ Type $^{\mathrm{d}}$} & \multirow{2}{*}{$\begin{array}{c}\text { Number of } \\
\text { spectra }^{\mathrm{e}}\end{array}$} \\
\hline & & & \multicolumn{2}{|c|}{ (degrees in J2000) } & & & \\
\hline 722 & 2005 ed & 0.0859 & 0.70569 & +0.75127 & 2005-09-04 & SN Ia & 1 \\
\hline 739 & $2005 \mathrm{ef}$ & 0.1072 & 14.59529 & +0.67906 & 2005-09-04 & SN Ia & 1 \\
\hline 744 & 2005 ei & 0.1286 & -30.80149 & +0.31750 & 2005-09-04 & SN Ia & 1 \\
\hline 762 & 2005 eg & 0.1912 & 15.53535 & -0.87899 & 2005-09-04 & SN Ia & 1 \\
\hline 774 & 2005 ex & 0.0936 & 25.46351 & -0.87638 & 2005-09-04 & SN Ia & 2 \\
\hline 1032 & $2005 \mathrm{ez}$ & 0.1299 & 46.79571 & +1.11953 & 2005-09-06 & SN Ia & 1 \\
\hline 1112 & $2005 \mathrm{fg}$ & 0.2585 & -20.98238 & -0.37519 & 2005-09-10 & SN Ia & 1 \\
\hline 1114 & $2005 \mathrm{lb}$ & 0.0257 & -16.29145 & -0.25250 & 2005-09-10 & SN II & 1 \\
\hline 1119 & $2005 \mathrm{fc}$ & 0.2982 & -39.58644 & +0.89466 & $2005-09-10$ & SN Ia & 1 \\
\hline 1166 & $\ldots$ & 0.3825 & 9.35564 & +0.97328 & 2005-09-10 & SN Ia & 1 \\
\hline 1241 & $2005 \mathrm{ff}$ & 0.089 & -22.32746 & -0.77659 & $2005-09-10$ & SN Ia & 2 \\
\hline 1253 & $2005 \mathrm{fd}$ & 0.2625 & -36.20095 & +0.16308 & $2005-09-10$ & SN Ia & 2 \\
\hline 1316 & $2005 \mathrm{fe}$ & 0.2174 & -25.13617 & +0.49444 & 2005-09-10 & SN Ia & 1 \\
\hline 1371 & $2005 \mathrm{fh}$ & 0.1189 & -10.62619 & +0.42939 & 2005-09-10 & SN Ia & 1 \\
\hline 1472 & 2005lc & 0.0144 & 45.54660 & -1.16651 & $2005-09-10$ & SN II & 1 \\
\hline 1580 & $2005 \mathrm{fb}$ & 0.186 & 45.32310 & -0.64409 & 2005-09-11 & SN Ia & 1 \\
\hline 1686 & $\ldots$ & 0.1367 & 2.24835 & -0.21039 & 2005-09-11 & SN Ia? & 1 \\
\hline 1688 & $\ldots$ & 0.3591 & -38.64223 & +0.32454 & 2005-09-11 & SN Ia & 1 \\
\hline 1794 & $2005 f j$ & 0.1426 & -42.16314 & -0.44537 & 2005-09-11 & SN Ia & 1 \\
\hline 2000 & $2005 \mathrm{hl}$ & 0.0243 & -46.16752 & +0.54298 & $2005-09-13$ & $\mathrm{SN} \mathrm{Ib}$ & 1 \\
\hline 2017 & 2005 fo & 0.2629 & -31.05666 & +0.59352 & 2005-09-13 & SN Ia & 1 \\
\hline 2030 & $2005 f l$ & 0.2343 & -48.15841 & -1.25328 & $2005-09-13$ & SN Ia & 1 \\
\hline 2031 & $2005 \mathrm{fm}$ & 0.1530 & -47.95680 & -1.17143 & $2005-09-13$ & SN Ia & 1 \\
\hline 2053 & $2005 \mathrm{fk}$ & 0.2651 & -41.16734 & -0.38295 & $2005-09-13$ & SN Ic & 1 \\
\hline 2102 & $2005 \mathrm{fn}$ & 0.0958 & -47.77902 & +0.19114 & $2005-09-13$ & SN Ia & 1 \\
\hline 2165 & $2005 \mathrm{fr}$ & 0.291 & 17.09174 & -0.09627 & 2005-09-14 & SN Ia & 1 \\
\hline 2246 & 2005 fy & 0.1952 & 50.09041 & -0.88558 & 2005-09-14 & SN Ia & 4 \\
\hline 2308 & 2005 ey & 0.149 & 34.27287 & +0.28030 & 2005-09-14 & SN Ia & 3 \\
\hline 2330 & $2005 \mathrm{fp}$ & 0.2135 & 6.80711 & +1.12060 & 2005-09-14 & SN Ia & 1 \\
\hline 2366 & $2005 f q$ & 0.1462 & 13.43351 & -0.56021 & $2005-09-15$ & SN II & 1 \\
\hline 2372 & $2005 \mathrm{ft}$ & 0.1798 & 40.52072 & -0.54085 & $2005-09-15$ & SN Ia & 3 \\
\hline 2422 & $2005 \mathrm{fi}$ & 0.268 & 1.99454 & +0.63820 & $2005-09-15$ & SN Ia & 1 \\
\hline 2440 & $2005 \mathrm{fu}$ & 0.194 & 42.63370 & +0.80780 & $2005-09-15$ & SN Ia & 1 \\
\hline 2533 & $2005 \mathrm{fs}$ & 0.344 & 31.22069 & -0.32647 & $2005-09-15$ & SN Ia & 1 \\
\hline 2561 & $2005 \mathrm{fv}$ & 0.1170 & 46.34345 & +0.85837 & $2005-09-15$ & SN Ia & 4 \\
\hline 2635 & $2005 \mathrm{fw}$ & 0.1440 & 52.70433 & -1.23808 & $2005-09-16$ & SN Ia & 3 \\
\hline 2661 & $\ldots$ & 0.1930 & -6.79249 & +0.09721 & $2005-09-16$ & SN II & 1 \\
\hline 2689 & $2005 \mathrm{fa}$ & 0.166 & 24.90035 & -0.75875 & $2005-09-16$ & SN Ia & 1 \\
\hline 2744 & $2005 \mathrm{hm}$ & 0.0350 & -35.24730 & -1.02741 & 2005-09-16 & $\mathrm{SN} \mathrm{Ib}$ & 1 \\
\hline 2789 & $2005 f x$ & 0.2907 & -15.79855 & +0.40110 & $2005-09-17$ & SN Ia & 1 \\
\hline 2916 & $2005 \mathrm{fz}$ & 0.121 & -44.07830 & +0.56956 & $2005-09-23$ & SN Ia & 4 \\
\hline 2943 & 2005 go & 0.2662 & 17.70495 & +1.00792 & $2005-09-24$ & SN Ia & 1 \\
\hline 2992 & $2005 \mathrm{gp}$ & 0.1266 & 55.49706 & -0.78267 & 2005-09-24 & SN Ia & 2 \\
\hline 3080 & $2005 \mathrm{ga}$ & 0.1739 & 16.93234 & -1.03950 & $2005-09-25$ & SN Ia & 3 \\
\hline 3087 & $2005 \mathrm{gc}$ & 0.1654 & 20.40671 & -0.97723 & $2005-09-25$ & SN Ia & 2 \\
\hline 3199 & $2005 \mathrm{gs}$ & 0.2511 & -26.70728 & +1.05054 & $2005-09-25$ & SN Ia & 1 \\
\hline 3241 & $2005 \mathrm{gh}$ & 0.387 & -47.34854 & -0.35409 & $2005-09-25$ & SN Ia? & 1 \\
\hline 3256 & $2005 \mathrm{hn}$ & 0.1076 & -30.73229 & -0.22348 & $2005-09-25$ & SN Ia & 1 \\
\hline 3317 & $2005 \mathrm{gd}$ & 0.1618 & 26.96261 & +0.64061 & $2005-09-25$ & SN Ia & 2 \\
\hline 3331 & 2005ge & 0.208 & 34.56138 & +0.79656 & $2005-09-25$ & SN Ia? & 2 \\
\hline 3377 & $2005 \mathrm{gr}$ & 0.2460 & 54.15620 & +1.07917 & $2005-09-25$ & SN Ia & 1 \\
\hline 3451 & $2005 \mathrm{gf}$ & 0.2498 & -25.93073 & +0.70815 & $2005-09-25$ & SN Ia & 1 \\
\hline 3452 & $2005 \mathrm{gg}$ & 0.2313 & -25.32850 & +0.63919 & $2005-09-25$ & SN Ia & 1 \\
\hline 3592 & $2005 \mathrm{gb}$ & 0.0870 & 19.05242 & +0.79195 & $2005-09-26$ & SN Ia & 3 \\
\hline 3818 & $2005 \mathrm{gi}$ & 0.0511 & 13.96950 & +0.50494 & $2005-09-26$ & SN II & 1 \\
\hline 3901 & 2005ho & 0.0638 & 14.85043 & +0.00261 & $2005-09-26$ & SN Ia & 2 \\
\hline 4000 & $2005 \mathrm{gt}$ & 0.2793 & 31.01601 & -0.36583 & $2005-09-26$ & SN Ia & 1 \\
\hline 4012 & $\ldots$ & 0.0253 & 48.44893 & -0.24360 & $2005-09-26$ & SN Ic & 1 \\
\hline 4046 & $2005 \mathrm{gw}$ & 0.278 & -5.50168 & +0.64211 & $2005-09-26$ & SN Ia & 1 \\
\hline 4241 & $2005 \mathrm{gu}$ & 0.3320 & 12.23769 & -0.90573 & $2005-09-27$ & SN Ia & 1 \\
\hline 4524 & $2005 \mathrm{gj}$ & 0.0618 & 45.29978 & -0.55387 & $2005-09-28$ & SN Ia & 19 \\
\hline 4577 & $2005 \mathrm{gv}$ & 0.365 & 38.47554 & +0.28071 & $2005-09-28$ & SN Ia & 1 \\
\hline 4679 & $2005 \mathrm{gy}$ & 0.3345 & 21.52834 & +0.67686 & 2005-09-29 & SN Ia & 1 \\
\hline 5103 & $2005 \mathrm{gx}$ & 0.148 & -0.11556 & +0.73717 & 2005-10-02 & SN Ia & 2 \\
\hline
\end{tabular}


Table 5

(Continued)

\begin{tabular}{|c|c|c|c|c|c|c|c|}
\hline \multirow{2}{*}{$\begin{array}{l}\text { Candidate } \\
\text { ID }^{\mathrm{a}}\end{array}$} & \multirow{2}{*}{$\begin{array}{c}\text { IAU } \\
\text { Name }\end{array}$} & \multirow[b]{2}{*}{$z^{\mathrm{b}}$} & R.A. ${ }^{c}$ & Decl. & \multirow{2}{*}{$\begin{array}{c}\text { Discovery } \\
\text { date }\end{array}$} & \multirow[b]{2}{*}{ Type $^{\mathrm{d}}$} & \multirow{2}{*}{$\begin{array}{c}\text { Number of } \\
\text { spectra }^{\mathrm{e}}\end{array}$} \\
\hline & & & \multicolumn{2}{|c|}{ (degrees in J2000) } & & & \\
\hline 5183 & $2005 \mathrm{gq}$ & 0.3905 & 53.45402 & +0.70935 & $2005-10-02$ & SN Ia & 1 \\
\hline 5350 & $2005 \mathrm{hp}$ & 0.1764 & -52.78083 & -0.77924 & $2005-10-03$ & SN Ia & 1 \\
\hline 5391 & $2005 \mathrm{hs}$ & 0.3009 & 52.34197 & -1.09470 & $2005-10-03$ & SN Ia & 1 \\
\hline 5395 & $2005 \mathrm{hr}$ & 0.1170 & 49.64095 & +0.12335 & $2005-10-03$ & SN Ia & 1 \\
\hline 5533 & $2005 \mathrm{hu}$ & 0.2206 & -31.33006 & +0.41343 & 2005-10-12 & SN Ia & 1 \\
\hline 5549 & $2005 h x$ & 0.120 & 3.25054 & +0.24823 & $2005-10-12$ & SN Ia & 1 \\
\hline 5550 & 2005hy & 0.1562 & 3.59830 & +0.33305 & $2005-10-12$ & SN Ia & 3 \\
\hline 5588 & 2005 hw & 0.4103 & 2.36862 & +1.15492 & $2005-10-12$ & SN Ia & 1 \\
\hline 5635 & $2005 \mathrm{hv}$ & 0.1795 & -26.81724 & -0.03492 & 2005-10-12 & SN Ia & 1 \\
\hline 5717 & $2005 i a$ & 0.2545 & 17.89596 & -0.00589 & $2005-10-12$ & SN Ia & 1 \\
\hline 5736 & $2005 \mathrm{jz}$ & 0.2654 & 22.86274 & -0.63162 & $2005-10-12$ & SN Ia & 1 \\
\hline 5737 & $2005 \mathrm{ib}$ & 0.3937 & 22.85704 & -0.60339 & $2005-10-12$ & SN Ia & 1 \\
\hline 5751 & $2005 \mathrm{hz}$ & 0.1305 & 11.63416 & +0.83823 & $2005-10-12$ & SN Ia & 3 \\
\hline 5821 & 2005hq & 0.465 & -47.41758 & -0.82528 & $2005-10-13$ & SN Ia? & 2 \\
\hline 5844 & $2005 \mathrm{ic}$ & 0.3118 & -32.21379 & -0.84294 & $2005-10-13$ & SN Ia & 1 \\
\hline 5916 & 2005 is & 0.1746 & 5.43739 & -0.32503 & $2005-10-13$ & SN Ia? & 3 \\
\hline 5944 & $2005 \mathrm{hc}$ & 0.0459 & 29.19980 & -0.21372 & $2005-10-13$ & SN Ia & 5 \\
\hline 5957 & $2005 i e$ & 0.2803 & 34.76058 & -0.27283 & $2005-10-13$ & SN Ia & 2 \\
\hline 5966 & 2005 it & 0.3090 & 16.19039 & +0.51392 & $2005-10-13$ & SN Ia & 1 \\
\hline 5994 & $2005 \mathrm{ht}$ & 0.189 & -47.39745 & -0.16789 & $2005-10-13$ & SN Ia & 1 \\
\hline 6057 & 2005 if & 0.0675 & 52.55362 & -0.97458 & $2005-10-15$ & SN Ia & 2 \\
\hline 6100 & $2005 \mathrm{ka}$ & 0.3186 & -26.51685 & +1.08649 & $2005-10-20$ & SN Ia & 1 \\
\hline 6108 & 2005 ih & 0.2603 & 1.80660 & +0.34898 & $2005-10-21$ & SN Ia & 1 \\
\hline 6127 & 2005 iw & 0.2894 & -22.67602 & -0.09236 & $2005-10-21$ & SN Ia & 1 \\
\hline 6137 & $2005 i v$ & 0.3001 & -52.06387 & +0.24475 & $2005-10-21$ & SN Ia & 1 \\
\hline 6192 & 2005jy & 0.2727 & -11.53501 & +1.25699 & $2005-10-21$ & SN Ia & 1 \\
\hline 6196 & $2005 \mathrm{ig}$ & 0.2809 & -22.36885 & -0.50266 & $2005-10-21$ & SN Ia & 1 \\
\hline 6249 & $2005 \mathrm{ii}$ & 0.2950 & 3.26555 & -0.62012 & $2005-10-21$ & SN Ia & 1 \\
\hline 6295 & 2005 js & 0.0793 & 23.67298 & -0.60537 & $2005-10-21$ & SN Ia & 2 \\
\hline 6304 & $2005 \mathrm{jk}$ & 0.185 & 26.49762 & +1.19596 & $2005-10-21$ & SN Ia & 3 \\
\hline 6315 & $2005 i x$ & 0.2557 & -49.51717 & +1.09200 & $2005-10-21$ & SN Ia & 2 \\
\hline 6406 & $2005 i j$ & 0.1240 & 46.08861 & -1.06296 & $2005-10-22$ & SN Ia & 2 \\
\hline 6422 & $2005 \mathrm{id}$ & 0.187 & -10.86119 & -0.66322 & $2005-10-22$ & SN Ia & 1 \\
\hline 6471 & $\ldots$ & 0.2018 & -50.61388 & +0.49495 & $2005-10-22$ & SN II & 1 \\
\hline 6558 & $2005 \mathrm{hj}^{\mathrm{f}}$ & 0.0574 & 21.70167 & -1.23812 & $2005-10-22$ & SN Ia & 2 \\
\hline 6649 & $2005 j \mathrm{~d}$ & 0.318 & 34.27586 & +0.53484 & $2005-10-23$ & SN Ia & 2 \\
\hline 6696 & $\ldots$ & 0.2382 & -20.92067 & +0.47925 & $2005-10-23$ & SN Ia? & 1 \\
\hline 6699 & $2005 \mathrm{ik}$ & 0.313 & -37.18502 & -1.05699 & $2005-10-23$ & SN Ia & 2 \\
\hline 6773 & 2005 iu & 0.0916 & -54.93498 & +0.21737 & $2005-10-24$ & SN Ia & 1 \\
\hline 6777 & 2005iy & 0.4043 & -38.78356 & +0.38561 & $2005-10-24$ & SN Ia & 2 \\
\hline 6780 & $2005 i z$ & 0.206 & -31.93139 & +0.26708 & $2005-10-24$ & SN Ia & 1 \\
\hline 6852 & 2005 jf & 0.3007 & 53.41983 & -0.11210 & $2005-10-24$ & SN Ia? & 1 \\
\hline 6924 & $2005 \mathrm{ja}$ & 0.329 & -1.03067 & +0.87698 & $2005-10-24$ & SN Ia & 1 \\
\hline 6933 & $2005 \mathrm{jc}$ & 0.214 & 11.35172 & +1.07558 & $2005-10-24$ & SN Ia & 2 \\
\hline 6936 & $2005 \mathrm{jl}$ & 0.1810 & -36.76618 & -0.69978 & $2005-10-24$ & SN Ia & 1 \\
\hline 6962 & $2005 \mathrm{je}$ & 0.0930 & 38.86091 & +1.07488 & $2005-10-24$ & SN Ia? & 1 \\
\hline 6968 & $\ldots$ & 0.0983 & 19.55569 & -0.90655 & $2005-10-24$ & SN Ia? & 3 \\
\hline 7017 & $\ldots$ & 0.1430 & 31.36534 & -0.49791 & $2005-10-24$ & SN Ia & 3 \\
\hline 7143 & $2005 j g$ & 0.306 & -14.73764 & -0.20737 & $2005-10-26$ & SN Ia & 1 \\
\hline 7147 & $2005 \mathrm{jh}$ & 0.1099 & -9.98155 & -0.05548 & $2005-10-26$ & SN Ia & 3 \\
\hline 7243 & $2005 \mathrm{jm}$ & 0.2045 & -31.92092 & +0.47193 & $2005-10-27$ & SN Ia & 1 \\
\hline 7335 & $2005 \mathrm{kn}$ & 0.197 & -41.11480 & -0.35534 & $2005-10-27$ & SN Ia? & 1 \\
\hline 7426 & $2005 \mathrm{jb}$ & 0.386 & -20.98663 & -0.36792 & $2005-10-27$ & SN Ia? & 1 \\
\hline 7460 & 2005jx & 0.2133 & -36.41594 & -0.68170 & $2005-10-27$ & SN Ia? & 1 \\
\hline 7473 & $2005 \mathrm{ji}$ & 0.220 & 4.32641 & -0.25726 & $2005-10-27$ & SN Ia & 2 \\
\hline 7475 & $2005 \mathrm{jn}$ & 0.3191 & 4.75348 & -0.28149 & $2005-10-27$ & SN Ia & 2 \\
\hline 7512 & 2005 jo & 0.2354 & 52.09032 & -0.32609 & $2005-10-27$ & SN Ia & 1 \\
\hline 7779 & $2005 \mathrm{jw}$ & 0.376 & -49.91979 & -0.00717 & $2005-10-28$ & SN Ia & 1 \\
\hline 7847 & $2005 j p$ & 0.2126 & 32.45995 & -0.06179 & $2005-10-28$ & SN Ia & 1 \\
\hline 7876 & $2005 \mathrm{ir}$ & 0.0767 & 19.18251 & +0.79463 & $2005-10-28$ & SN Ia & 2 \\
\hline 7947 & $2005 \mathrm{jj}$ & 0.368 & -45.81392 & +0.40835 & $2005-10-28$ & SN Ia? & 1 \\
\hline 8030 & $2005 \mathrm{jv}$ & 0.423 & 40.20883 & +0.99314 & $2005-10-28$ & SN Ia & 1 \\
\hline 8046 & $2005 \mathrm{ju}$ & 0.2591 & 39.11682 & +0.51126 & 2005-10-29 & SN Ia & 1 \\
\hline
\end{tabular}


Table 5

(Continued)

\begin{tabular}{|c|c|c|c|c|c|c|c|}
\hline \multirow{2}{*}{$\begin{array}{l}\text { Candidate } \\
\text { ID }^{\mathrm{a}}\end{array}$} & \multirow{2}{*}{$\begin{array}{c}\text { IAU } \\
\text { Name }\end{array}$} & \multirow[b]{2}{*}{$z^{\mathrm{b}}$} & R.A. ${ }^{c}$ & Decl. & \multirow{2}{*}{$\begin{array}{c}\text { Discovery } \\
\text { date }\end{array}$} & \multirow[b]{2}{*}{ Type $^{\mathrm{d}}$} & \multirow{2}{*}{$\begin{array}{l}\text { Number of } \\
\text { spectra }^{\mathrm{e}}\end{array}$} \\
\hline & & & \multicolumn{2}{|c|}{ (degrees in J2000) } & & & \\
\hline 8151 & $2005 \mathrm{hk}$ & 0.0131 & 6.96200 & -1.19815 & $2005-10-29$ & SN Ia & 11 \\
\hline 8213 & $2005 \mathrm{ko}$ & 0.1855 & -2.47907 & -0.92139 & $2005-10-31$ & SN Ia & 2 \\
\hline 8495 & $2005 \mathrm{mi}$ & 0.2151 & -24.73898 & -0.74816 & $2005-10-31$ & SN Ia & 1 \\
\hline 8598 & $2005 \mathrm{jt}$ & 0.3613 & 42.66734 & -0.06596 & 2005-11-01 & SN Ia & 1 \\
\hline 8679 & $2005 \mathrm{jr}$ & 0.2945 & 15.05152 & +0.34081 & 2005-11-01 & SN II & 1 \\
\hline 8707 & $2005 \mathrm{mh}$ & 0.3951 & 41.23617 & +0.20359 & 2005-11-01 & SN Ia? & 1 \\
\hline 8719 & $2005 \mathrm{kp}$ & 0.1174 & 7.72148 & -0.71884 & 2005-11-01 & SN Ia & 2 \\
\hline 8921 & 2005ld & 0.1462 & -34.99800 & -0.00792 & 2005-11-02 & SN Ia & 2 \\
\hline 9032 & 2005le & 0.2549 & -22.11544 & -0.49357 & 2005-11-02 & SN Ia & 1 \\
\hline 9045 & $2005 \mathrm{kq}$ & 0.3903 & -12.16286 & -0.60863 & 2005-11-02 & SN Ia & 1 \\
\hline 9118 & $2005 \mathrm{kr}$ & 0.1344 & 47.12358 & +0.88893 & $2005-11-03$ & SN Ic & 1 \\
\hline 9207 & $2005 \lg$ & 0.3509 & 19.08363 & -0.80780 & $2005-11-03$ & SN Ia & 1 \\
\hline 9273 & $2005 \mathrm{ks}$ & 0.0992 & -35.51432 & -0.03245 & $2005-11-03$ & SN Ic & 1 \\
\hline 9457 & 2005li & 0.2572 & -24.18564 & +0.25304 & 2005-11-04 & SN Ia & 1 \\
\hline 9467 & $20051 \mathrm{~h}$ & 0.2191 & -31.04860 & +1.18079 & 2005-11-04 & SN Ia & 2 \\
\hline 10028 & $2005 \mathrm{kt}$ & 0.0662 & 17.74185 & +0.27615 & 2005-11-07 & SN Ia & 1 \\
\hline 10096 & $20051 \mathrm{j}$ & 0.0789 & 29.42928 & -0.17945 & 2005-11-07 & SN Ia & 1 \\
\hline 10106 & $\ldots$ & 0.1479 & 47.69327 & -0.20488 & 2005-11-07 & SN Ia? & 2 \\
\hline 10297 & $2005 \mathrm{mk}$ & 0.1486 & -19.33066 & +0.83301 & 2005-11-08 & SN II & 1 \\
\hline 10367 & $2005 \mathrm{~kb}$ & 0.0155 & 12.71115 & +0.85362 & 2005-11-08 & SN II & 2 \\
\hline 10434 & $2005 \mathrm{lk}$ & 0.1037 & -30.04406 & -1.19371 & 2005-11-08 & SN Ia & 1 \\
\hline 10449 & 200511 & 0.246 & -22.97138 & -1.12817 & 2005-11-08 & SN Ia & 1 \\
\hline 10550 & $20051 f$ & 0.3008 & -10.32462 & -1.20487 & 2005-11-08 & SN Ia & 1 \\
\hline 10805 & $2005 \mathrm{ku}$ & 0.0456 & -15.07246 & -0.01371 & $2005-11-10$ & SN Ia & 1 \\
\hline 10907 & $20051 \mathrm{~m}$ & 0.0853 & 3.77046 & +0.35514 & 2005-11-12 & SN II & 1 \\
\hline 11017 & $2005 \mathrm{mj}{ }^{\mathrm{g}}$ & 0.210 & -37.04401 & -1.06939 & $2005-11-13$ & SN II & 1 \\
\hline 11067 & $2005 \mathrm{ml}$ & 0.119 & 33.51840 & -0.23918 & 2005-11-14 & SN Ia & 1 \\
\hline 11206 & $2005 \mathrm{~mm}$ & 0.3805 & 3.28979 & +1.14552 & $2005-11-20$ & SN Ia & 1 \\
\hline 11300 & $2005 \ln$ & 0.130 & 6.75050 & -0.58662 & $2005-11-20$ & SN Ia & 1 \\
\hline 11320 & $2005 \mathrm{mo}$ & 0.2743 & 57.55376 & -0.24022 & $2005-11-22$ & SN Ia & 1 \\
\hline 11332 & $2005 \mathrm{mn}$ & 0.0474 & 57.32682 & -0.69207 & $2005-11-23$ & $\mathrm{SN} \mathrm{Ib}$ & 4 \\
\hline 11452 & 2005lo & 0.300 & 9.29943 & -1.20342 & $2005-11-24$ & SN Ia & 1 \\
\hline 11557 & $20051 \mathrm{q}$ & 0.382 & 40.40017 & +0.20502 & $2005-11-24$ & SN Ia & 1 \\
\hline 11650 & $2005 \mathrm{mp}$ & 0.275 & 16.19032 & +0.05563 & $2005-11-25$ & SN Ia & 1 \\
\hline 11864 & $20051 \mathrm{p}$ & 0.305 & 26.92832 & +0.20722 & $2005-11-26$ & SN Ia & 1 \\
\hline 12136 & $2005 \mathrm{mq}$ & 0.352 & -9.90923 & -0.34989 & $2005-11-27$ & SN Ia & 1 \\
\hline
\end{tabular}

Notes.

${ }^{a}$ Internal SN candidate designation.

${ }^{\mathrm{b}}$ Final spectroscopic redshifts. When narrow galaxy lines are detected in at least one of the spectra, the redshifts are given to four decimal places. The typical uncertainties are $\sim 0.0005$. When only the $\mathrm{SN}$ is detected, the redshifts are given to three decimal places and the uncertainties are $\sim 0.005$ (see Zheng et al. 2007).

${ }^{\mathrm{c}}$ Right ascension is provided in decimal degrees defined in the range $\left[-180^{\circ},+180^{\circ}\right]$.

d The final spectroscopic type of the SN candidate. Spectroscopically probable SNe Ia are designated "SN Ia?" (see Zheng et al. 2007).

e This includes all spectral observations performed including those of the SN candidate and possibly its host galaxy.

${ }^{\mathrm{f}}$ Spectroscopically confirmed by Quimby et al. (2007).

g Spectroscopically confirmed by the ESSENCE Group.

(This table is also available in a machine-readable form in the online journal)

\section{RESULTS FROM FALL 2005 AND 2006}

The 2005 season resulted in 73 unique SDSS imaging runs acquired on 59 different nights. Approximately half of the frames were taken under non-photometric conditions, bright moon, and/or poor seeing. A total of 155,616 objects were visually inspected during this season, of which 24,402 were tagged as potential $\mathrm{SNe}$, resulting in 11,385 unique $\mathrm{SN}$ candidates (see Table 7). Interestingly, 6618 of those candidates were detected in only a single epoch, and are mostly likely moving solar system objects that were not filtered properly. A few of these singleepoch candidates may be fast transients that rise and fade on timescales shorter than $\sim 1$ day (Becker et al. 2004). Most of the remaining 4767 candidates are true variables or transient sources. ${ }^{46}$ A short summary of the 2005 and 2006 seasons is shown in Table 7.

For the 2005 search season, we acquired 248 spectra from 187 distinct $\mathrm{SN}$ candidates under various observing conditions. A total of 130 unique candidates were spectroscopically confirmed to be of type Ia. One of these SNe Ia (SN2005hj) was co-discovered

\footnotetext{
46 A very small fraction of the 4767 multi-epoch candidates are artifacts of image subtraction, e.g., faint dipoles that appear in the same place at two or more different epochs.
} 
Table 6

List of Spectroscopically Confirmed Supernovae in 2006

\begin{tabular}{|c|c|c|c|c|c|c|c|}
\hline \multirow{2}{*}{$\begin{array}{l}\text { Candidate } \\
\text { ID }^{\mathrm{a}}\end{array}$} & \multirow{2}{*}{$\begin{array}{c}\text { IAU } \\
\text { Name }\end{array}$} & \multirow[b]{2}{*}{$z^{\mathrm{b}}$} & R.A. ${ }^{c}$ & Decl. & \multirow{2}{*}{$\begin{array}{c}\text { Discovery } \\
\text { date }\end{array}$} & \multirow[b]{2}{*}{ Type $^{\mathrm{d}}$} & \multirow{2}{*}{$\begin{array}{c}\text { Number of } \\
\text { spectra }^{\mathrm{e}}\end{array}$} \\
\hline & & & \multicolumn{2}{|c|}{ (degrees in J2000) } & & & \\
\hline 12778 & $2006 \mathrm{fs}$ & 0.10 & -42.50417 & +0.40879 & 2006-08-27 & SN Ia & 1 \\
\hline 12779 & $2006 f d$ & 0.08 & -50.52813 & +1.22112 & $2006-08-27$ & SN Ia & 1 \\
\hline 12780 & $2006 \mathrm{eq}^{\mathrm{f}}$ & 0.05 & -37.84531 & +1.22811 & $2006-08-27$ & SN Ia & 2 \\
\hline 12781 & 2006er & 0.08 & 5.40656 & -1.00997 & $2006-08-27$ & SN Ia & 1 \\
\hline 12782 & $2006 f q$ & 0.07 & 5.00338 & -0.62497 & $2006-08-27$ & SN II & 1 \\
\hline 12819 & 2006ho & 0.11 & 8.36686 & -0.32024 & 2006-08-29 & SN II & 1 \\
\hline 12820 & $2006 f g$ & 0.04 & 9.58353 & -0.29499 & 2006-08-29 & SN II & 4 \\
\hline 12841 & $2006 \mathrm{gk}$ & 0.29 & -38.65275 & -1.03367 & 2006-09-11 & SN Ia & 1 \\
\hline 12842 & $2006 \mathrm{ez}$ & 0.09 & -36.54476 & -1.01596 & 2006-09-11 & SN II & 4 \\
\hline 12843 & $2006 f a$ & 0.17 & -36.12191 & -0.98027 & 2006-09-11 & SN Ia & 2 \\
\hline 12851 & $2006 \mathrm{gm}$ & 0.25 & -15.21893 & -0.62737 & 2006-09-11 & SN Ia & 1 \\
\hline 12853 & 2006еy & 0.17 & -43.23444 & +0.72307 & 2006-09-11 & SN Ia & 1 \\
\hline 12855 & $2006 \mathrm{fk}$ & 0.17 & -29.74447 & +0.71624 & 2006-09-11 & SN Ia & 2 \\
\hline 12856 & $2006 f l$ & 0.17 & -27.13453 & +0.75598 & 2006-09-11 & SN Ia & 1 \\
\hline 12860 & $2006 \mathrm{fc}$ & 0.12 & -36.30580 & +1.17591 & 2006-09-11 & SN Ia & 2 \\
\hline 12869 & 2006ge & 0.28 & -32.60088 & +0.00108 & 2006-09-12 & SN Ia & 1 \\
\hline 12874 & $2006 \mathrm{fb}$ & 0.24 & -6.03538 & -0.17711 & 2006-09-12 & SN Ia & 1 \\
\hline 12881 & 2006gu & 0.24 & 10.16235 & -0.07357 & 2006-09-12 & SN Ia & 1 \\
\hline 12883 & $2006 \mathrm{fr}$ & 0.31 & -47.37411 & +0.39867 & 2006-09-12 & SN Ia? & 1 \\
\hline 12897 & $2006 \mathrm{eb}$ & 0.02 & 18.42139 & -0.10158 & 2006-09-12 & SN Ia & 1 \\
\hline 12898 & $2006 \mathrm{fw}$ & 0.08 & 26.79308 & -0.14696 & 2006-09-12 & SN Ia & 3 \\
\hline 12907 & $2006 \mathrm{fv}$ & 0.13 & 20.40784 & +0.41453 & 2006-09-12 & SN Ia? & 1 \\
\hline 12927 & $2006 f j$ & 0.19 & 41.78614 & +0.77513 & 2006-09-12 & SN Ia & 1 \\
\hline 12928 & 2006ew & 0.14 & -54.99097 & -0.97569 & 2006-09-12 & SN Ia & 1 \\
\hline 12930 & $2006 \mathrm{ex}$ & 0.15 & -50.31722 & -0.47453 & 2006-09-12 & SN Ia & 1 \\
\hline 12950 & 2006fy & 0.08 & -8.33255 & -0.84028 & 2006-09-12 & SN Ia & 2 \\
\hline 12971 & $2006 f f$ & 0.23 & 6.64850 & -0.30207 & 2006-09-12 & SN Ia & 1 \\
\hline 12972 & $2006 \mathrm{ft}$ & 0.26 & 7.95859 & -0.38296 & 2006-09-12 & SN Ia & 1 \\
\hline 12977 & $2006 \mathrm{gh}$ & 0.25 & 13.69561 & -0.25086 & 2006-09-12 & SN Ia & 1 \\
\hline 12979 & $2006 \mathrm{gf}$ & 0.12 & 11.60148 & +0.00348 & 2006-09-12 & SN Ia & 1 \\
\hline 12983 & $2006 \mathrm{gl}$ & 0.27 & 16.45878 & +0.14480 & 2006-09-12 & SN Ia & 1 \\
\hline 12991 & $2006 \mathrm{gd}$ & 0.15 & 17.60995 & -1.06803 & 2006-09-12 & SN II & 1 \\
\hline 13005 & 2006fh & 0.12 & 27.39546 & -0.64988 & 2006-09-12 & SN Ia & 1 \\
\hline 13025 & $2006 f x$ & 0.22 & -18.43267 & +0.41590 & 2006-09-15 & SN Ia & 2 \\
\hline 13038 & 2006gn & 0.10 & -12.17322 & +0.50461 & 2006-09-15 & SN Ia & 1 \\
\hline 13044 & $2006 \mathrm{fm}$ & 0.12 & -27.45725 & +0.50326 & 2006-09-15 & SN Ia & 3 \\
\hline 13045 & $2006 f n$ & 0.18 & -14.97506 & +0.53767 & 2006-09-15 & SN Ia & 1 \\
\hline 13070 & $2006 f u$ & 0.20 & -2.21494 & -0.74634 & 2006-09-15 & SN Ia & 1 \\
\hline 13072 & $2006 f i$ & 0.23 & -25.04053 & +0.02437 & 2006-09-15 & SN Ia & 2 \\
\hline 13099 & $2006 \mathrm{gb}$ & 0.27 & -0.18127 & -1.25038 & 2006-09-16 & SN Ia & 1 \\
\hline 13135 & $2006 f z$ & 0.10 & 4.17229 & -0.42454 & 2006-09-16 & SN Ia & 9 \\
\hline 13136 & 2006go & 0.37 & 6.14068 & -0.27906 & 2006-09-16 & SN Ia & 1 \\
\hline 13152 & $2006 \mathrm{gg}$ & 0.20 & 7.05211 & +0.11801 & 2006-09-16 & SN Ia & 1 \\
\hline 13174 & $2006 \mathrm{ga}$ & 0.24 & 13.23469 & +0.44786 & 2006-09-16 & SN Ia & 2 \\
\hline 13195 & 2006 fo & 0.02 & 38.16203 & +0.61751 & 2006-09-16 & SN Ic & 11 \\
\hline 13254 & $2006 \mathrm{gx}$ & 0.18 & 42.05867 & -0.34703 & 2006-09-16 & SN Ia & 1 \\
\hline 13305 & 2006he & 0.21 & -28.89951 & +0.69121 & 2006-09-17 & SN Ia & 1 \\
\hline 13327 & 2006jf & 0.28 & -21.27261 & +0.00204 & 2006-09-17 & SN Ia & 1 \\
\hline 13354 & $2006 \mathrm{hr}$ & 0.16 & 27.56482 & -0.88727 & 2006-09-17 & SN Ia & 1 \\
\hline 13355 & 2006kh & 0.06 & 27.29935 & -0.60526 & 2006-09-17 & SN II & 1 \\
\hline 13357 & $2006 \mathrm{gw}$ & 0.33 & 29.47294 & -0.53415 & 2006-09-17 & SN Ia & 1 \\
\hline 13370 & $2006 \mathrm{gv}$ & 0.20 & 27.15155 & +0.32822 & 2006-09-18 & SN Ia & 1 \\
\hline 13376 & $2006 \mathrm{gq}$ & 0.07 & 26.30225 & +0.35644 & 2006-09-18 & SN II & 4 \\
\hline 13425 & $2006 g p$ & 0.21 & -21.45832 & +0.05499 & 2006-09-18 & SN Ia & 1 \\
\hline 13449 & 2006ix & 0.08 & -0.19154 & -0.31104 & 2006-09-18 & SN II & 1 \\
\hline 13467 & 2006hq & 0.07 & -5.05354 & +0.20523 & 2006-09-18 & SN Ia? & 1 \\
\hline 13506 & 2006hg & 0.24 & 25.24334 & -0.72785 & 2006-09-19 & SN Ia & 1 \\
\hline 13511 & 2006hh & 0.24 & 40.61235 & -0.79412 & 2006-09-19 & SN Ia & 1 \\
\hline 13518 & $2006 \mathrm{mn}^{\mathrm{g}}$ & $\ldots$ & 16.95163 & -0.10984 & 2006-09-19 & SN Ia & $\ldots$ \\
\hline 13577 & $2006 \mathrm{~kg}$ & 0.23 & 16.07075 & +0.76913 & 2006-09-19 & SN II & 1 \\
\hline 13578 & 2006hc & 0.20 & 17.39470 & +0.70416 & 2006-09-19 & SN Ia & 1 \\
\hline 13610 & 2006hd & 0.30 & -33.98522 & +0.72628 & 2006-09-19 & SN Ia & 1 \\
\hline
\end{tabular}


Table 6

(Continued)

\begin{tabular}{|c|c|c|c|c|c|c|c|}
\hline \multirow{2}{*}{$\begin{array}{l}\text { Candidate } \\
\text { ID }^{\mathrm{a}}\end{array}$} & \multirow{2}{*}{$\begin{array}{c}\text { IAU } \\
\text { Name }\end{array}$} & \multirow[b]{2}{*}{$z^{\mathrm{b}}$} & R.A. ${ }^{c}$ & Decl. & \multirow{2}{*}{$\begin{array}{c}\text { Discovery } \\
\text { date }\end{array}$} & \multirow[b]{2}{*}{ Type $^{\mathrm{d}}$} & \multirow{2}{*}{$\begin{array}{c}\text { Number of } \\
\text { spectra }^{\mathrm{e}}\end{array}$} \\
\hline & & & \multicolumn{2}{|c|}{ (degrees in J2000) } & & & \\
\hline 13641 & 2006hf & 0.22 & -14.78127 & -0.98114 & 2006-09-19 & SN Ia & 1 \\
\hline 13655 & 2006hs & 0.26 & 39.02022 & -0.99418 & 2006-09-19 & SN Ia & 1 \\
\hline 13727 & 2006hj & 0.23 & -42.41192 & +0.93262 & 2006-09-20 & SN Ia & 2 \\
\hline 13736 & $2006 \mathrm{hv}$ & 0.15 & -23.16671 & +1.03097 & $2006-09-20$ & SN Ia & 2 \\
\hline 13757 & $2006 \mathrm{hk}$ & 0.28 & -9.87691 & -1.15785 & 2006-09-20 & SN Ia & 1 \\
\hline 13796 & 2006hl & 0.15 & -9.30843 & +0.53288 & 2006-09-20 & SN Ia & 3 \\
\hline 13830 & $2006 \mathrm{hm}$ & 0.33 & -8.43828 & -0.38228 & 2006-09-20 & SN Ia & 1 \\
\hline 13835 & 2006hp & 0.25 & 6.05964 & -0.24826 & 2006-09-20 & SN Ia & 1 \\
\hline 13894 & $2006 \mathrm{jh}$ & 0.13 & 1.69061 & -0.03676 & 2006-09-22 & SN Ia & 1 \\
\hline 13934 & 2006jg & 0.33 & -17.88899 & -0.43527 & 2006-09-22 & SN Ia & 1 \\
\hline 13936 & $2006 \mathrm{hz}$ & 0.23 & 3.17083 & -0.53548 & 2006-09-22 & SN II & 1 \\
\hline 13956 & 2006hi & 0.26 & 20.94181 & +0.81673 & $2006-09-23$ & SN Ia & 1 \\
\hline 14019 & 2006ki & 0.22 & -43.35758 & -0.64795 & $2006-09-27$ & SN Ia & 1 \\
\hline 14024 & 2006ht & 0.20 & -41.80144 & +0.91635 & $2006-09-27$ & SN Ia & 1 \\
\hline 14108 & 2006hu & 0.14 & 53.59465 & -1.12321 & $2006-09-28$ & SN Ia & 1 \\
\hline 14157 & $2006 \mathrm{kj}$ & 0.21 & 51.13669 & +1.02236 & 2006-09-28 & SN Ia? & 1 \\
\hline 14212 & 2006iy & 0.20 & -29.52989 & +1.04501 & 2006-09-29 & SN Ia & 1 \\
\hline 14261 & $2006 \mathrm{jk}$ & 0.29 & -31.75960 & +0.25379 & 2006-09-29 & SN Ia & 1 \\
\hline 14279 & $2006 h x$ & 0.04 & 18.48878 & +0.37167 & 2006-09-29 & SN Ia & 6 \\
\hline 14284 & $2006 i b$ & 0.18 & 49.04929 & -0.60094 & 2006-09-29 & SN Ia & 1 \\
\hline 14298 & $2006 \mathrm{jj}$ & 0.27 & -45.10495 & +1.22326 & 2006-09-29 & SN Ia & 1 \\
\hline 14318 & 2006py & 0.06 & -19.57480 & -0.13692 & 2006-09-30 & SN Ia & 3 \\
\hline 14331 & $2006 \mathrm{kl}$ & 0.22 & 7.88868 & -0.13581 & 2006-09-30 & SN Ia & 1 \\
\hline 14377 & 2006hw & 0.14 & 48.26431 & -0.47163 & 2006-09-30 & SN Ia & 1 \\
\hline 14397 & $2006 \mathrm{kk}$ & 0.39 & 6.91610 & +0.64951 & 2006-09-30 & SN Ia & 1 \\
\hline 14421 & $2006 i a$ & 0.17 & 31.82988 & +1.25208 & 2006-09-30 & SN Ia & 1 \\
\hline 14437 & 2006hy & 0.14 & -27.91904 & -1.19639 & 2006-10-01 & SN Ia & 2 \\
\hline 14450 & $2006 \mathrm{kn}$ & 0.12 & -45.47827 & +0.90048 & 2006-10-01 & SN II & 1 \\
\hline 14451 & 2006ji & 0.18 & -51.80505 & +0.92707 & 2006-10-01 & SN Ia & 1 \\
\hline 14456 & $2006 \mathrm{jm}$ & 0.33 & -16.44890 & +1.05066 & 2006-10-01 & SN Ia & 1 \\
\hline 14481 & $20061 \mathrm{j}$ & 0.24 & 2.68173 & +0.20131 & 2006-10-01 & SN Ia & 1 \\
\hline 14492 & 2006jo & 0.08 & 20.81130 & -0.32964 & 2006-10-01 & SN Ib & 2 \\
\hline 14599 & 2006j1 & 0.05 & -51.98055 & -0.57454 & 2006-10-01 & SN II & 4 \\
\hline 14735 & $2006 \mathrm{~km}$ & 0.30 & 35.15803 & +0.34838 & 2006-10-02 & SN Ia & 1 \\
\hline 14782 & 2006jp & 0.16 & -45.76575 & -0.27904 & 2006-10-02 & SN Ia & 1 \\
\hline 14815 & $2006 i z$ & 0.14 & -40.92836 & +0.55949 & 2006-10-03 & SN Ia & 3 \\
\hline 14816 & 2006ja & 0.11 & -23.28370 & +0.50606 & 2006-10-03 & SN Ia & 3 \\
\hline 14846 & 2006jn & 0.23 & 7.66324 & +0.14158 & 2006-10-03 & SN Ia & 1 \\
\hline 14871 & 2006jq & 0.11 & 54.27710 & +0.00936 & $2006-10-03$ & SN Ia & 3 \\
\hline 14979 & $2006 \mathrm{jr}$ & 0.18 & 54.94643 & +0.99282 & $2006-10-03$ & SN Ia & 1 \\
\hline 14984 & $2006 j \mathrm{~s}$ & 0.19 & -46.16643 & -0.09279 & 2006-10-03 & SN Ia & 1 \\
\hline 15002 & $2006 \mathrm{ko}$ & 0.38 & 22.24981 & +0.76995 & $2006-10-04$ & SN Ia? & 1 \\
\hline 15009 & $2006 \mathrm{kp}$ & 0.29 & 32.79856 & +0.69583 & 2006-10-04 & SN Ia? & 1 \\
\hline 15031 & 2006iw & 0.03 & -9.66882 & +0.25973 & 2006-10-04 & SN II & 5 \\
\hline 15057 & $2006 \mathrm{md}^{\mathrm{g}}$ & $\ldots$ & 17.88126 & 0.40949 & 2006-10-04 & SN Ia & $\ldots$ \\
\hline 15129 & $2006 \mathrm{kq}$ & 0.20 & -41.09761 & -0.32143 & 2006-10-04 & SN Ia & 1 \\
\hline 15132 & $2006 \mathrm{jt}$ & 0.14 & -30.29977 & +0.19769 & 2006-10-04 & SN Ia & 2 \\
\hline 15136 & 2006ju & 0.15 & -8.83745 & -0.71836 & 2006-10-05 & SN Ia? & 1 \\
\hline 15161 & 2006jw & 0.25 & 35.84291 & +0.81900 & 2006-10-12 & SN Ia? & 1 \\
\hline 15170 & $2006 j x$ & 0.25 & 58.05858 & +0.29207 & 2006-10-12 & $\mathrm{SN} \mathrm{Ib}$ & 1 \\
\hline 15171 & $2006 \mathrm{~kb}$ & 0.12 & -55.20746 & -1.06449 & 2006-10-13 & SN Ia & 2 \\
\hline 15201 & $2006 \mathrm{ks}$ & 0.21 & -22.48059 & +0.00364 & 2006-10-13 & SN Ia & 1 \\
\hline 15203 & 2006jy & 0.20 & 15.73471 & +0.18309 & $2006-10-13$ & SN Ia & 1 \\
\hline 15213 & $2006 \mathrm{lk}$ & 0.31 & 53.01914 & -0.10014 & 2006-10-13 & SN Ia & 1 \\
\hline 15217 & $2006 j v$ & 0.37 & 22.63421 & +0.21980 & 2006-10-13 & SN Ia & 1 \\
\hline 15219 & $2006 \mathrm{ka}$ & 0.25 & 34.61115 & +0.22669 & 2006-10-13 & SN Ia & 1 \\
\hline 15222 & $2006 j z$ & 0.20 & 2.85338 & +0.70274 & 2006-10-13 & SN Ia & 1 \\
\hline 15229 & $2006 \mathrm{kr}$ & 0.22 & 4.83198 & +1.09072 & 2006-10-13 & SN Ia & 1 \\
\hline 15234 & $2006 \mathrm{kd}$ & 0.13 & 16.95834 & +0.82820 & 2006-10-13 & SN Ia & 2 \\
\hline 15254 & 2006oy & 0.20 & -46.50709 & -0.36005 & 2006-10-14 & SN Ia & 1 \\
\hline 15259 & $2006 \mathrm{kc}$ & 0.22 & -22.45580 & -0.40782 & $2006-10-14$ & SN Ia & 1 \\
\hline
\end{tabular}


Table 6

(Continued)

\begin{tabular}{|c|c|c|c|c|c|c|c|}
\hline \multirow{2}{*}{$\begin{array}{l}\text { Candidate } \\
\text { ID }^{\mathrm{a}}\end{array}$} & \multirow{2}{*}{$\begin{array}{c}\text { IAU } \\
\text { Name }\end{array}$} & \multirow[b]{2}{*}{$z^{\mathrm{b}}$} & R.A. ${ }^{c}$ & Decl. & \multirow{2}{*}{$\begin{array}{c}\text { Discovery } \\
\text { date }\end{array}$} & \multirow[b]{2}{*}{ Type $^{\mathrm{d}}$} & \multirow{2}{*}{$\begin{array}{c}\text { Number of } \\
\text { spectra }^{\mathrm{e}}\end{array}$} \\
\hline & & & \multicolumn{2}{|c|}{ (degrees in $\mathbf{J} 2000$ ) } & & & \\
\hline 15286 & $\ldots g$ & 0.00 & -56.60836 & -1.11915 & 2006-10-15 & SN Ia & $\ldots$ \\
\hline 15287 & $2006 \mathrm{kt}$ & 0.23 & -36.04032 & -1.05743 & 2006-10-15 & SN Ia & 1 \\
\hline 15301 & 2006lo & 0.18 & -36.42008 & +0.58911 & 2006-10-15 & SN Ia & 1 \\
\hline 15320 & $2006 \mathrm{kv}$ & 0.06 & 0.77773 & +0.91377 & 2006-10-16 & SN II & 4 \\
\hline 15339 & $2006 n s$ & 0.12 & -36.61574 & +0.78484 & 2006-10-17 & SN II & 4 \\
\hline 15340 & $2006 \mathrm{mu}$ & 0.14 & -23.99717 & +0.82135 & 2006-10-17 & SN Ia? & 1 \\
\hline 15354 & $20061 p$ & 0.22 & 6.77368 & -0.12598 & 2006-10-17 & SN Ia & 2 \\
\hline 15356 & $20061 \mathrm{~m}$ & 0.28 & -24.94668 & +0.40995 & 2006-10-17 & SN Ia & 1 \\
\hline 15365 & $2006 \mathrm{ku}$ & 0.19 & -5.44334 & +1.24909 & 2006-10-18 & SN Ia & 1 \\
\hline 15369 & $20061 n$ & 0.24 & -11.16705 & -0.56261 & 2006-10-18 & SN Ia & 2 \\
\hline 15383 & 2006lq & 0.32 & 34.14953 & -0.15516 & 2006-10-18 & SN Ia & 1 \\
\hline 15421 & $2006 \mathrm{kw}$ & 0.18 & 33.74160 & +0.60251 & 2006-10-21 & SN Ia & 1 \\
\hline 15425 & $2006 \mathrm{kx}$ & 0.16 & 55.56108 & +0.47829 & 2006-10-21 & SN Ia & 1 \\
\hline 15433 & $2006 \mathrm{mt}$ & 0.22 & 14.87963 & -0.25651 & 2006-10-21 & SN Ia & 2 \\
\hline 15440 & $20061 \mathrm{r}$ & 0.26 & 39.72067 & +0.09013 & 2006-10-21 & SN Ia & 1 \\
\hline 15443 & $20061 b$ & 0.18 & 49.86744 & -0.31803 & 2006-10-21 & SN Ia & 1 \\
\hline 15453 & $2006 \mathrm{ky}$ & 0.18 & -40.33170 & -1.02429 & 2006-10-21 & SN Ia & 1 \\
\hline 15456 & 200611 & 0.38 & -28.13274 & -0.90341 & 2006-10-21 & SN Ia & 1 \\
\hline 15459 & 2006la & 0.12 & -19.29859 & -0.90175 & 2006-10-21 & SN Ia & 1 \\
\hline 15461 & $2006 \mathrm{kz}$ & 0.18 & -33.15252 & -0.49468 & 2006-10-21 & SN Ia & 1 \\
\hline 15466 & $2006 \mathrm{mz}$ & 0.25 & -42.35496 & -0.12322 & 2006-10-21 & SN Ia & 1 \\
\hline 15475 & $20061 \mathrm{c}$ & 0.02 & -18.89802 & -0.16487 & 2006-10-21 & SN Ic & 7 \\
\hline 15504 & 2006 oc & 0.27 & -14.29855 & -0.87642 & $2006-10-22$ & SN Ia & 1 \\
\hline 15508 & $20061 \mathrm{~s}$ & 0.14 & 27.16898 & -0.57661 & 2006-10-22 & SN Ia & 3 \\
\hline 15557 & $2006 \mathrm{oz}$ & 0.00 & -27.77686 & +0.89735 & $2006-10-23$ & $\mathrm{SN} \mathrm{Ib}$ & 1 \\
\hline 15583 & $2006 \mathrm{mv}$ & 0.17 & 37.73108 & +0.94630 & $2006-10-23$ & SN Ia & 1 \\
\hline 15584 & $2006 n t$ & 0.28 & 43.49546 & +0.98696 & 2006-10-23 & SN Ia & 1 \\
\hline 15648 & 2006ni & 0.17 & -46.28162 & -0.19482 & $2006-10-23$ & SN Ia & 1 \\
\hline 15674 & 2006nu & 0.20 & -19.17084 & +0.26291 & $2006-10-23$ & SN Ia & 1 \\
\hline 15704 & 2006nh & 0.36 & 40.21073 & +0.65875 & 2006-10-24 & SN Ia & 1 \\
\hline 15734 & 2006ng & 0.40 & 5.53028 & -0.09564 & 2006-10-24 & SN Ia & 1 \\
\hline 15749 & $2006 \mathrm{mw}$ & 0.12 & 51.28531 & -0.04058 & 2006-10-24 & SN Ia? & 1 \\
\hline 15756 & $2006 n f$ & 0.39 & 0.37483 & +0.27597 & 2006-10-24 & SN Ia & 1 \\
\hline 15760 & $2006 \mathrm{mh}^{\mathrm{g}}$ & $\ldots$ & 18.80583 & 0.39925 & 2006-10-24 & SN Ia & $\ldots$ \\
\hline 15776 & 2006na & 0.32 & 32.82944 & -0.99826 & 2006-10-24 & SN Ia & 1 \\
\hline 15833 & $2006 m x$ & 0.13 & -59.13613 & +0.57021 & $2006-10-26$ & SN Ia? & 1 \\
\hline 15868 & 2006pa & 0.25 & 38.09980 & -0.71358 & 2006-10-28 & SN Ia? & 3 \\
\hline 15872 & $2006 n b$ & 0.21 & 36.72250 & -0.32781 & 2006-10-28 & SN Ia & 1 \\
\hline 15897 & $2006 \mathrm{pb}$ & 0.17 & 11.68145 & -1.03290 & 2006-10-29 & SN Ia & 1 \\
\hline 15901 & 2006od & 0.17 & 31.97630 & -0.53539 & 2006-10-29 & SN Ia & 1 \\
\hline 16000 & 2006nj & 0.40 & 21.11762 & +0.07439 & 2006-10-30 & SN Ia & 1 \\
\hline 16021 & $2006 \mathrm{nc}$ & 0.12 & 13.84373 & -0.38881 & $2006-10-30$ & SN Ia & 2 \\
\hline 16032 & 2006nk & 0.20 & 44.06922 & -0.41077 & $2006-10-30$ & SN Ia & 1 \\
\hline 16069 & 2006nd & 0.13 & -18.75392 & -1.00660 & 2006-10-31 & SN Ia & 4 \\
\hline 16072 & $2006 n v$ & 0.29 & 3.12444 & -0.97731 & 2006-10-31 & SN Ia & 1 \\
\hline 16073 & 2006of & 0.16 & 8.10776 & -1.05400 & 2006-10-31 & SN Ia & 1 \\
\hline 16087 & $2006 \mathrm{pc}$ & 0.06 & 26.04409 & -0.15589 & 2006-11-01 & SN II & 1 \\
\hline 16093 & 2006oe & 0.34 & -9.63753 & +1.13248 & 2006-11-01 & SN Ia & 1 \\
\hline 16099 & $2006 n n$ & 0.20 & 26.42094 & -1.05439 & 2006-11-01 & SN Ia & 1 \\
\hline 16100 & 2006nl & 0.19 & 30.43629 & -1.03241 & 2006-11-01 & SN Ia & 1 \\
\hline 16106 & 2006no & 0.25 & -27.91065 & -1.14878 & 2006-11-01 & SN Ia & 1 \\
\hline 16108 & 2006 og & 0.16 & -56.98224 & +0.57061 & 2006-11-01 & SN Ia & 1 \\
\hline 16116 & 2006oh & 0.16 & -4.69799 & -1.10581 & 2006-11-01 & SN Ia & 1 \\
\hline 16165 & 2006nw & 0.16 & 30.73314 & -0.53387 & 2006-11-02 & SN Ia & 1 \\
\hline 16179 & $2006 n x$ & 0.14 & 53.37764 & -0.67729 & 2006-11-03 & SN Ic & 6 \\
\hline 16185 & 2006ok & 0.10 & 16.86807 & -0.26937 & 2006-11-09 & SN Ia & 1 \\
\hline 16192 & 2006ny & 0.08 & 9.82211 & +0.08393 & 2006-11-09 & SN II & 2 \\
\hline 16206 & 2006pe & 0.16 & 5.78819 & -0.05363 & 2006-11-09 & SN Ia & 1 \\
\hline 16211 & $2006 \mathrm{~nm}$ & 0.31 & -11.83591 & +0.26693 & 2006-11-09 & SN Ia & 1 \\
\hline 16213 & 2006 oi & 0.20 & 8.97105 & +0.25840 & 2006-11-09 & SN Ia & 1 \\
\hline
\end{tabular}


Table 6

(Continued)

\begin{tabular}{|c|c|c|c|c|c|c|c|}
\hline \multirow{2}{*}{$\begin{array}{l}\text { Candidate } \\
\text { ID }^{\mathrm{a}}\end{array}$} & \multirow{2}{*}{$\begin{array}{c}\text { IAU } \\
\text { Name }\end{array}$} & \multirow[b]{2}{*}{$z^{\mathrm{b}}$} & R.A. ${ }^{c}$ & Decl. & \multirow{2}{*}{$\begin{array}{c}\text { Discovery } \\
\text { date }\end{array}$} & \multirow[b]{2}{*}{ Type $^{\mathrm{d}}$} & \multirow{2}{*}{$\begin{array}{c}\text { Number of } \\
\text { spectra }^{\mathrm{e}}\end{array}$} \\
\hline & & & \multicolumn{2}{|c|}{ (degrees in J2000) } & & & \\
\hline 16215 & 2006ne & 0.05 & 18.40767 & +0.42388 & 2006-11-09 & SN Ia & 8 \\
\hline 16232 & $2006 o j$ & 0.34 & 17.20541 & -0.98955 & $2006-11-10$ & SN Ia & 1 \\
\hline 16259 & 2006ol & 0.12 & -7.97003 & +0.85635 & $2006-11-10$ & SN Ia & 1 \\
\hline 16276 & 2006om & 0.16 & 20.57866 & +1.01062 & $2006-11-10$ & SN Ia & 1 \\
\hline 16280 & $2006 n z$ & 0.04 & 14.12170 & -1.22673 & 2006-11-11 & SN Ia & 4 \\
\hline 16281 & 2006pd & 0.19 & -7.05785 & -0.66805 & 2006-11-11 & SN Ia & 1 \\
\hline 16287 & $2006 n p$ & 0.11 & 46.66451 & +0.06403 & 2006-11-11 & SN Ia & 6 \\
\hline 16314 & $20060 a$ & 0.06 & -39.07107 & -0.84347 & $2006-11-12$ & SN Ia & 3 \\
\hline 16333 & 2006on & 0.07 & -31.00630 & -1.07020 & 2006-11-12 & SN Ia & 3 \\
\hline 16350 & $2006 \mathrm{ph}$ & 0.24 & -36.36051 & -0.88583 & 2006-11-14 & SN Ia & 1 \\
\hline 16352 & $2006 \mathrm{pk}$ & 0.25 & -13.75064 & -0.87336 & 2006-11-14 & SN Ia & 2 \\
\hline 16357 & 2006pi & 0.37 & -29.18082 & -0.60684 & 2006-11-14 & SN Ia & 1 \\
\hline 16392 & $2006 \mathrm{ob}$ & 0.06 & 27.95060 & +0.26331 & 2006-11-14 & SN Ia & 6 \\
\hline 16402 & $2006 s v$ & 0.30 & -23.63228 & +0.26527 & 2006-11-14 & SN Ia & 2 \\
\hline 16414 & 2006pj & 0.31 & -20.67354 & +0.73814 & 2006-11-14 & SN Ia & 1 \\
\hline 16421 & 2006pg & 0.32 & 51.83630 & +1.08966 & 2006-11-14 & SN Ia & 1 \\
\hline 16442 & 200600 & 0.28 & -30.05319 & -0.73322 & $2006-11-15$ & SN Ia & 1 \\
\hline 16473 & 2006pl & 0.22 & -31.38785 & +0.58852 & 2006-11-15 & SN Ia & 2 \\
\hline 16482 & $2006 \mathrm{pm}$ & 0.20 & -31.29049 & +0.93367 & 2006-11-15 & SN Ia & 1 \\
\hline 16541 & $2006 \mathrm{pn}$ & 0.13 & 57.26188 & -0.53277 & $2006-11-16$ & SN Ia & 1 \\
\hline 16567 & $2006 \mathrm{pf}$ & 0.40 & 25.43805 & -0.18915 & 2006-11-17 & SN Ia & 1 \\
\hline 16578 & 2006po & 0.18 & -54.18680 & -0.84849 & 2006-11-17 & SN Ia & 1 \\
\hline 16618 & $2006 \mathrm{pq}$ & 0.20 & 21.27937 & -1.21894 & 2006-11-18 & SN Ia & 1 \\
\hline 16619 & $2006 \mathrm{ps}$ & 0.10 & 25.93874 & -1.11192 & $2006-11-18$ & SN Ia & 2 \\
\hline 16631 & $2006 p v$ & 0.21 & 54.62279 & -0.67557 & 2006-11-18 & SN Ia & 1 \\
\hline 16641 & $2006 \mathrm{pr}$ & 0.11 & 23.56123 & -0.40363 & 2006-11-18 & SN Ia & 3 \\
\hline 16644 & $2006 \mathrm{pt}$ & 0.30 & 36.81737 & -0.39348 & 2006-11-18 & SN Ia & 1 \\
\hline 16668 & $2006 \mathrm{pu}$ & 0.15 & 40.72425 & +0.89287 & 2006-11-18 & SN II & 1 \\
\hline 16692 & 2006op & 0.03 & -39.61722 & +0.99331 & 2006-11-18 & SN Ia? & 2 \\
\hline 16737 & $2006 \mathrm{qc}$ & 0.20 & -47.16732 & -0.95232 & 2006-11-20 & SN Ia & 1 \\
\hline 16748 & $2006 s x$ & 0.23 & 7.95132 & -0.51394 & 2006-11-20 & SN Ia & 2 \\
\hline 16758 & 2006pw & 0.33 & -18.38765 & +1.15081 & 2006-11-20 & $\mathrm{SN}$ Ia & 1 \\
\hline 16774 & 2006 sy & 0.21 & 35.27168 & +0.82840 & $2006-11-20$ & SN Ia & 2 \\
\hline 16776 & $2006 q d$ & 0.27 & -12.97859 & -0.17608 & 2006-11-20 & SN Ia & 1 \\
\hline 16779 & $2006 q a$ & 0.40 & 45.29054 & -0.02624 & 2006-11-20 & $\mathrm{SN}$ Ia & 1 \\
\hline 16781 & $2006 q b$ & 0.33 & 52.06103 & -0.20096 & 2006-11-20 & SN Ia & 1 \\
\hline 16789 & $2006 \mathrm{pz}$ & 0.33 & 43.78434 & +0.23477 & $2006-11-20$ & SN Ia & 1 \\
\hline 16793 & 2006qg & 0.23 & -31.42464 & +0.44701 & 2006-11-20 & SN Ia & 1 \\
\hline 16836 & 2006sw & 0.33 & 16.25085 & -0.37464 & 2006-11-21 & SN Ia & 1 \\
\hline 16840 & $2006 \mathrm{qf}$ & 0.17 & 57.98849 & -0.39658 & 2006-11-21 & SN II & 1 \\
\hline 16847 & $2006 p x$ & 0.28 & -6.67875 & +0.96110 & 2006-11-21 & SN Ia & 1 \\
\hline 16872 & $2006 \mathrm{qh}$ & 0.12 & -33.02908 & +0.70684 & 2006-11-21 & SN Ia & 1 \\
\hline 16894 & $2006 \mathrm{tb}$ & 0.30 & 56.31453 & -0.12854 & 2006-11-22 & $\mathrm{SN}$ Ia & 1 \\
\hline 16899 & $2006 \mathrm{ta}$ & 0.29 & 52.46008 & +0.29509 & $2006-11-22$ & SN Ia & 1 \\
\hline 16938 & 2006qe & 0.39 & -11.91674 & -0.53442 & $2006-11-22$ & SN Ia & 1 \\
\hline 16941 & $2006 s z$ & 0.19 & -4.28142 & -0.55636 & $2006-11-22$ & SN Ia? & 1 \\
\hline 16953 & 2006pp & 0.34 & 12.24364 & +0.47695 & $2006-11-23$ & SN Ia & 1 \\
\hline 16956 & $2006 q j$ & 0.11 & -57.22662 & -0.31996 & $2006-11-23$ & SN Ia & 1 \\
\hline 16988 & $2006 \mathrm{qk}$ & 0.06 & -23.61507 & +0.15419 & 2006-11-24 & SN Ic & 4 \\
\hline 17048 & 2006qi & 0.19 & 27.58151 & +0.88490 & 2006-11-24 & SN Ia & 1 \\
\hline 17081 & 2006ql & 0.27 & 27.53797 & +0.42048 & $2006-11-25$ & SN Ia & 1 \\
\hline 17106 & $2006 \mathrm{tc}$ & 0.21 & 47.00818 & +0.94343 & $2006-11-25$ & SN Ia & 1 \\
\hline 17117 & $2006 \mathrm{qm}$ & 0.14 & 40.60031 & -0.79652 & $2006-11-25$ & SN Ia & 2 \\
\hline 17135 & $2006 r z$ & 0.03 & 56.52825 & +0.38975 & $2006-11-26$ & SN Ia & 4 \\
\hline
\end{tabular}

Notes.

a Internal SN candidate designation.

${ }^{\mathrm{b}}$ Preliminary spectroscopic redshifts. The typical uncertainties are $\sim 0.01$.

${ }^{c}$ Right ascension is provided in decimal degrees defined in the range $\left[-180^{\circ},+180^{\circ}\right]$.

d The preliminary spectroscopic type of the SN candidate. Spectroscopically probable SNe Ia are designated "SN Ia?" (see Zheng et al. 2007).

e This includes all spectral observations performed including those of the SN candidate and possibly its host galaxy.

f Spectroscopically confirmed by the CfA SN Group.

g Spectroscopically confirmed by the ESSENCE Group.

(This table is also available in a machine-readable form in the online journal) 
Table 7

Summary of the 2005 and 2006 Search Seasons

\begin{tabular}{lcc}
\hline \hline Number of & 2005 & 2006 \\
\hline Nights on SDSS $2.5 \mathrm{~m}$ & 59 & 60 \\
Runs & 73 & 90 \\
Total number of objects & 375,187 & 391,435 \\
$\quad$ Number of objects scanned & 155,616 & 14,430 \\
$\quad$ Number of objects tagged as SN & 24,402 & 4,189 \\
& 11,385 & 3,694 \\
SN candidates discovered $^{\mathrm{a}}$ & 6,618 & 599 \\
Single-epoch candidates $^{\mathrm{b}}$ & &
\end{tabular}

Notes.

a The number of unique SN candidates tagged by a scanner. This number differs from the number of objects tagged as a SN because it includes multiple objects from the same candidate.

${ }^{\mathrm{b}}$ Candidates that were detected in only a single run. Most of these sources are likely to be solar system objects.

and spectroscopically observed by the Texas Supernova Search (Quimby et al. 2007). The numbers of confirmed core-collapse $\mathrm{SNe}$ were 7 and 13 for type Ib/c and II, respectively. One of these type II SNe (SN2005mj) was spectroscopically confirmed by the ESSENCE Group. An additional 40 candidates were observed in the post-season during 2005 December-2006 January. A sample of 41 SN targets including SN2005hk (Phillips et al. 2007) and SN2005gj (Prieto et al. 2007) for which we acquired 16 and 9 spectra, respectively, were observed spectroscopically more than once. The host galaxy of one 2005 candidate (SN7017) was observed during the 2006 search season. Interestingly, that supernova was still active and visible a year after discovery; the spectrum was identified as a peculiar SN similar to SN2002ic and SN2005gj.

In 2006, 90 SDSS imaging runs were taken on 60 nights. The number of visually-inspected objects was reduced by more than an order of magnitude to 14,430 . This improvement is due to the modified moving object finder and the choice of scanning only second-epoch and bright first-epoch objects. We identified a total of 3694 unique SN candidates. Surprisingly, there is still a somewhat large sample (599 out of 3694) of slow-moving single-epoch objects that were tagged by a scanner as a possible $\mathrm{SN}$ and made the candidate list.

During the second search season, a total of 449 spectra were acquired, $\sim 80 \%$ more than the 2005 season, from 285 unique candidates. Though the majority of these were observations of new 2006 candidates, a handful of host galaxies of the 2005 candidates were observed as well. A sample of 197 candidates, including 5 candidates that were observed by the CfA SN Group and the ESSENCE Survey, were spectroscopically confirmed to be SNe Ia and 14 candidates were identified as spectroscopically probable SNe Ia. The numbers of confirmed core-collapse SNe were 7 and 19 for type Ib/c and II, respectively.

Table 8 summarizes the spectroscopic observations grouped into candidates discovered in 2005 and 2006. We performed 172 (241) observations of new SN Ia candidates discovered in 2005 (2006), of which 130 (197) were spectroscopically confirmed to be SNe Ia, and 16 (14) candidates were identified as spectroscopically probable $\mathrm{SNe} \mathrm{Ia}$; the latter are denoted SN Ia? in Tables 5 and 6. In addition, observations of 7 (7) additional candidates in 2005 (2006) resulted in galaxy spectra (either the $\mathrm{SN}$ had faded or the galaxy was too bright relative to the $\mathrm{SN}$ ) with spectroscopic redshifts consistent with photometric redshifts estimated from the multi-band light curves of the SNe. Therefore, in the first two years, $90 \%$ of SN Ia
Table 8

Spectroscopic Observations of 2005 and 2006 Candidates

\begin{tabular}{|c|c|c|}
\hline Number of Spectroscopic Observations & 2005 & 2006 \\
\hline SN Ia candidates ${ }^{\mathrm{a}}$ & 173 & 241 \\
\hline Identified as SN Ia & $130^{\mathrm{b}}$ & $197^{\mathrm{c}}$ \\
\hline Identified as probable SN Ia & 16 & 14 \\
\hline Resulting in host galaxy spectra of photometric SN Ia & 7 & 7 \\
\hline Resulting in other host galaxy spectra & 3 & 6 \\
\hline Identified as $\mathrm{SN} \mathrm{Ib/c}$ & 1 & 0 \\
\hline Identified as SN II & $5^{\mathrm{d}}$ & 6 \\
\hline Identified as AGN & 2 & 0 \\
\hline Identified as flaring M-dwarf & 1 & 1 \\
\hline Unidentified & 8 & 10 \\
\hline Non-SN Ia candidates ${ }^{\mathrm{e}}$ & 24 & 42 \\
\hline Identified as $\mathrm{SN} \mathrm{Ib/c}$ & 7 & 6 \\
\hline Identified as SN II & 7 & 14 \\
\hline Photometric SN Ia candidates with host galaxy spectroscopy ${ }^{f}$ & 81 & 6 \\
\hline Other SN candidates with host galaxy spectroscopyg & 26 & 0 \\
\hline Duplicate and multi-epoch spectra ${ }^{\mathrm{h}}$ & 86 & 148 \\
\hline Total & 390 & 437 \\
\hline
\end{tabular}

Notes.

a Number of candidates targeted for spectroscopy that had been photometrically identified as a possible SN Ia.

b This includes one SN Ia that was spectroscopically confirmed by the Texas Supernova Search (Quimby et al. 2007).

c This includes five SNe Ia that were spectroscopically confirmed by the CfA SN Group and the ESSENCE Group.

${ }^{d}$ This includes one SN II that was spectroscopically confirmed by the ESSENCE Group.

e This includes observations of core-collapse SNe, novae, and other interesting transients.

${ }^{f}$ Of the 113 host galaxy spectra obtained, the measured redshifts of a sample of 81 sources were consistent with the photometric redshift estimated from the SN Ia model light curve fits, and were thus identified as photometric SN Ia.

$\mathrm{g}$ These were targeted as host galaxies of photometric SN Ia candidates. The observations, however, resulted in either noisy, unclassifiable spectra or measured spectroscopic redshifts that are inconsistent with the SN Ia light curve redshifts implying that the candidates are not SNe Ia.

$\mathrm{h}$ The total number of duplicate and multi-epoch spectra; not the number of candidates targeted for multi-epoch spectroscopy.

targets resulted in spectra of SNe Ia, probable $\mathrm{SNe}$ Ia, or host galaxies of photometric SN Ia candidates. A total of $12 \mathrm{SN}$ Ia targets $(3 \%)$ were classified instead as core-collapse $\mathrm{SNe}$, 4 targets $(1 \%)$ were classified as either an AGN or a flaring M-dwarf, and 9 targets (2\%) resulted in galaxy spectra with spectroscopic redshifts inconsistent with photometric redshifts estimated from the SN Ia model fits. Finally, there is a sample of 18 spectra (4\%), most of which were taken under poor weather conditions, that are unidentified.

Evaluation of the full light curves after the end of each search season enables identification of additional photometric SN Ia candidates which, for various reasons, were not spectroscopically observed during the search. We have identified at least 200 high-quality SN Ia candidates from the 2005 candidate list, and have already acquired host galaxy spectra for a significantly-sized subsample. These targets are also good fillers during periods of poor observing conditions at APO that result in a lack of good new SN candidates. As of the writing of this paper, we have measured redshifts for 81 host galaxies of candidates with Ia-like light curves. An additional 13 candidates were identified to be good Ia candidates with host galaxy redshifts from the SDSS redshift survey. 


\section{SUMMARY}

The search pipeline of the SDSS-II SN survey has enabled efficient discoveries of variable and transient astronomical sources, filtering over 375,000 objects detected each season into several thousands of SN candidates. A reliable photometric SN typing system and spectroscopic follow-up algorithm have allowed spectroscopic observations of $\sim 150$ SNe Ia per season with $90 \%$ targeting efficiency. After two seasons, the search and spectroscopic follow-up algorithms have reached a relatively mature stage, and it is unlikely that major changes will be made for the third and final season of 2007. If the observing conditions resemble those of the previous two years, we expect to increase our sample of spectroscopically confirmed SN Ia by an additional $\sim 150-200$ events, reaching a sample of $\sim 500$ confirmed SNe Ia for the completed survey. A majority of the low-redshift sources will have well-sampled multi-band light curves that can serve as templates for future studies.

Spectroscopic target selection by a human observer is appropriate (and preferred) for a relatively small survey like the SDSS-II SN survey, but this is unlikely to be feasible for future large-scale surveys that will discover thousands or tens of thousands of SNe over the period of a few years. Based on our experience, however, we believe that much of the candidate identification process can be automated, and with just 2-4 epochs of multi-band imaging, SN candidates can be assigned probabilities that are reliable enough for performing follow-up spectroscopy. More quantitative studies of SN identification using photometric data alone will be presented in a future article.

Funding for the SDSS and SDSS-II has been provided by the Alfred P. Sloan Foundation, the Participating Institutions, the National Science Foundation, the U.S. Department of Energy, the National Aeronautics and Space Administration, the Japanese Monbukagakusho, the Max Planck Society, and the Higher Education Funding Council for England. The SDSS web site is http://www.sdss.org/.

The SDSS is managed by the Astrophysical Research Consortium for the Participating Institutions. The Participating Institutions are the American Museum of Natural History, Astrophysical Institute Potsdam, University of Basel, Cambridge University, Case Western Reserve University, University of Chicago, Drexel University, Fermilab, the Institute for Advanced Study, the Japan Participation Group, Johns Hopkins University, the Joint Institute for Nuclear Astrophysics, the Kavli Institute for Particle Astrophysics and Cosmology, the Korean Scientist Group, the Chinese Academy of Sciences (LAMOST), Los Alamos National Laboratory, the Max Planck Institute for Astronomy (MPIA), the Max Planck Institute for Astrophysics (MPA), New Mexico State University, Ohio State University, University of Pittsburgh, University of Portsmouth, Princeton University, the United States Naval Observatory, and the University of Washington.

The Hobby-Eberly Telescope (HET) is a joint project of the University of Texas at Austin, the Pennsylvania State University, Stanford University, Ludwig-Maximillians-Universität München, and Georg-August-Universität Göttingen. The HET is named in honor of its principal benefactors, William P. Hobby and Robert E. Eberly. The Marcario Low-Resolution Spectrograph is named for Mike Marcario of High Lonesome Optics, who fabricated several optics for the instrument but died before its completion; it is a joint project of the Hobby-Eberly
Telescope partnership and the Instituto de Astronomía de la Universidad Nacional Autónoma de México. The Apache Point Observatory $3.5 \mathrm{~m}$ telescope is owned and operated by the Astrophysical Research Consortium. We thank the observatory director, Suzanne Hawley, and site manager, Bruce Gillespie, for their support of this project. The Subaru Telescope is operated by the National Astronomical Observatory of Japan. The William Herschel Telescope is operated by the Isaac Newton Group, and the Nordic Optical Telescope is operated jointly by Denmark, Finland, Iceland, Norway, and Sweden, both on the island of La Palma in the Spanish Observatorio del Roque de los Muchachos of the Instituto de Astrofisica de Canarias. Observations at the ESO New Technology Telescope at La Silla Observatory were made under programme IDs 77.A-0437, 78.A-0325, and 79.A-0715. Kitt Peak National Observatory, National Optical Astronomy Observatory, is operated by the Association of Universities for Research in Astronomy, Inc. (AURA) under cooperative agreement with the National Science Foundation. The WIYN Observatory is a joint facility of the University of Wisconsin-Madison, Indiana University, Yale University, and the National Optical Astronomy Observatories. The W.M. Keck Observatory is operated as a scientific partnership among the California Institute of Technology, the University of California, and the National Aeronautics and Space Administration. The Observatory was made possible by the generous financial support of the W.M. Keck Foundation. The South African Large Telescope of the South African Astronomical Observatory is operated by a partnership between the National Research Foundation of South Africa, Nicolaus Copernicus Astronomical Center of the Polish Academy of Sciences, the Hobby-Eberly Telescope Board, Rutgers University, Georg-August-Universität Göttingen, University of Wisconsin-Madison, University of Canterbury, University of North Carolina-Chapel Hill, Dartmough College, Carnegie Mellon University, and the United Kingdom SALT consortium.

\section{APPENDIX A}

\section{SUPERNOVA SOFTWARE}

This appendix describes the details of the supernova software that runs on the computer cluster at APO.

The majority of Framesub is written in the Perl language. This provides the internal glue that strings together the various processing steps. In general, the image-level computations are written in the $C$ language. These applications are called by the Perl scripts. The implementation of Framesub on the computing cluster is controlled by shell scripts.

As a programmatic summary, the Framesub pipeline consists of a series of stages, each of which has actions which it undertakes, as well as dependencies on the successful completion of previous stages. By default, an ensemble of images is passed from stage to stage using input and output lists. If an image fails the assigned actions in a particular stage, the frame is not passed into the input list for the subsequent stages of processing. A record is kept of the fact that it failed, and all such failures must be addressed individually. Below we describe the three modes in which the pipeline is run.

\section{A1. sdssred}

This mode performs the basic reduction and preparation of the data for image subtraction.

FINDNEWIM. After processing by Photo, Framesub must initialize its input list of images. This stage searches for 
the correct data products ${ }^{47}$ for the camera column-filter combination assigned to it. This includes the $\mathrm{fpC}$ science image, a fpM mask image, an asTrans file providing the astrometry, and a psField file that contains Photo's model of the PSF (Stoughton et al. 2002).

MKSATMASK. The fpM file contains a pixel-by-pixel mask corresponding to any operations that have happened on the science image. This includes interpolation over CCD defects and cosmic rays, the masking of saturated pixels, and interpolation over bleed trails from the saturated stars. This stage translates the SDSS-format mask file into a mask image understood by Framesub. In practice, this is merely a reassignment to the numerical values of the mask bits.

We provide an additional level of editing in these images by masking halos around clusters of saturated pixels, as well as extending diffraction spike masks at the orientation correct for the camera rotation. These additional masks are only added for clusters of more than 20 saturated pixels. An operational scale length $r$ for the halo and diffraction masking is determined by using the number of saturated pixels $N_{\text {sat }}$ and solving for $r$ through $N_{\text {sat }}=\pi r^{2}$.

SDSSPSF. This stage converts the solution for the PSF obtained by Photo contained in the psField files, into a format readable by Dophot (Schechter et al. 1993). The Photo PSF solution is later used to make an aperture correction to the difference image photometric measurements (see Section A3).

SDSSSTARS. This stage obtains a list of the positions, magnitudes, and magnitude errors for the calibration stars contained within the frame and stores them in a text file suitable as input to SDSSZERO, which computes the zeropoints of each of the frames (see below). The list of calibration stars is also an optional input to DIFFIM, providing a list of preferred sources to be used in deriving the optimal convolution kernel (see Section A3). The magnitudes and magnitude errors were initially based on the DR4 database (Adelman-McCarthy et al. 2006), but were later updated based on the catalog provided by Ivezić et al. (2007).

The difference images obtained by the SN Survey are always photometrically scaled to the template image; the following two stages are included when running sdssred on the template images.

STARDOPHOT. This stage performs doPhot photometry on a list of calibration stars provided by SDSSSTARS, using the modified version of the Dophot software package.

SDSSZERO. This stage performs zeropointing of the frame. The magnitudes and magnitude errors of the calibration stars are first converted to flux units via $10^{-0.4 \mathrm{~m}}$, and then the model, ADU counts $=\mathrm{A}^{*}$ flux, is solved for A using the weighted least-squares procedure. The zeropointing algorithm does iterative sigma clipping at $\{24.0,12.0,6.0,6.0,6$.$\} standard deviations from the best-$ fit zeropoint model. The final zeropoint, the statistical error on the zeropoint, and the RMS of the zeropoint are then converted into magnitudes and stored for use during the $\mathrm{SN}$ search.

\footnotetext{
${ }^{47}$ http://www.sdss.org/dr6/dm/flatFiles/FILES.html.
}

\section{A2. sdssred With Fakes}

To monitor the software and human search efficiencies, we insert artificial $\mathrm{SNe}$ (fakes) into the data stream in real-time. Prior to the beginning of the survey, a library of fakes was generated (1000 fakes in 2005). Each fake is assigned a redshift, celestial position $(\alpha, \delta)$, date of peak-magnitude in $V$ band, and an intrinsic luminosity. The redshift distribution was generated according to $d N / d z \propto z^{2}$ and the celestial positions are then chosen to be near to a galaxy with the same redshift, based on a catalog of photometric redshifts (Oyaizu et al. 2007).

The fakes generated during the 2005 observing campaign did not include extinction and reddening; however, the set of fakes generated during the 2006 campaign included extinction, with $A_{V}$ chosen from an exponential distribution, $P\left(A_{V}\right) \propto e^{-A_{V} / 0.4}$.

The following stages are used to generate realistic type Ia SN light curves for the fakes in real time.

SDSSFAKESELECT. To minimize computation time for inserting fakes into the data stream and still retain the ability to simulate the magnitude at an arbitrary light-curve epoch, a range of dates for which the fake magnitude is within the expected limit of the SDSS telescope was computed. This stage checks the library of fakes to see whether any fake has a position within the limits of the field and that the present MJD falls within the expected range of observability.

STARDOPHOT_FAKE. This stage performs Dophot photometry on calibration stars within the frame. This is necessary to dynamically derive the magnitude to ADU conversion for the fakes.

SDSSZERO_FAKE. This stage computes a zeropoint for the frame, based on the dophot photometry from the previous stage. It is identical to the SDSSZERO stage described in Section A1.

SDSSFAKEINSERT. This stage inserts the fake SNe into the search image. Given the current MJD and the fake light-curve parameters described above, the theoretical magnitude is computed in real-time. The light-curve model is identical to the stretch model used for online photometric typing, as described in Section 3.1. Given the magnitude and the zeropoint computed above, an integrated flux value is computed. The PSF model for the fake comes from the Photo derived PSF; this is remapped to the astrometric grid of the search image and photometrically scaled. Poisson noise is added to each pixel. Finally, the unperturbed search image is copied and the ADU values for each pixel are overlaid on the search image, which is passed to the subsequent processing stages. A record of changes to the image is written to the $\mathrm{fpC}$ file header, and the magnitudes and corresponding MJD are written to a MySQL database.

\section{A3. sdssdiff}

This operational mode works with two sets of images that have been successfully run through the sdssred stages. One set of data is considered the "template" for difference imaging, and they are subtracted from the science images to yield only those objects that have varied in position or brightness.

In practice, most of our template images consist of the PSFoptimized co-adds of SDSS Stripe 82 (Annis et al. 2006). The images consist of 5-10 co-added exposures, depending on the position in the stripe, from runs prior to the 2005 SN search season. Only images with seeing $\lesssim 1.1^{\prime \prime}$ are used. Figure 9 


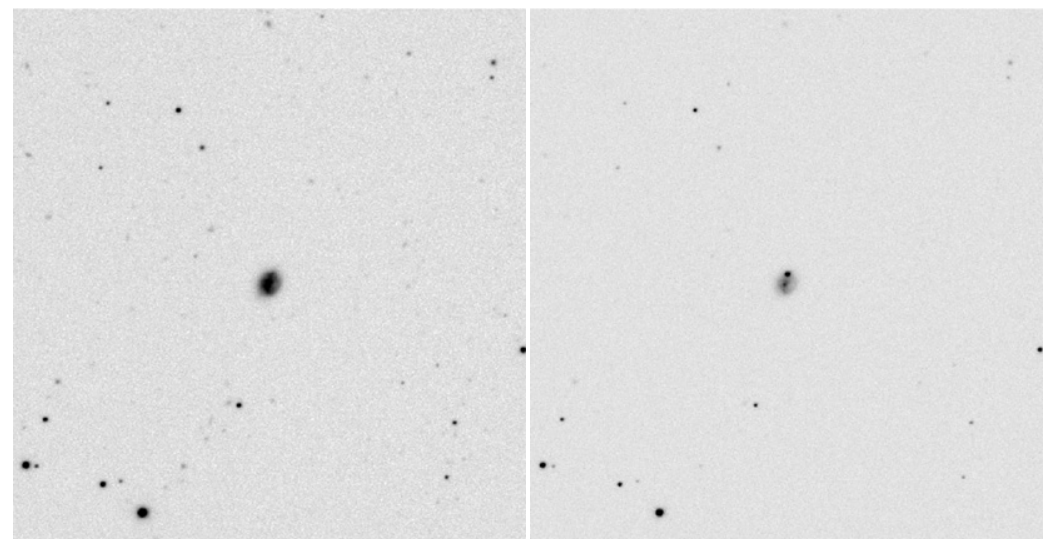

Figure 9. Template (left) and search (right) $r$-band images centered on the host galaxy of SN2005ir, a type Ia SN at $z=0.076$. The SN is visible above and slightly to the right of the of the host galaxy in the right-hand image. The search image was part of run 5823, and was taken under photometric conditions with $\sim 1.1^{\prime \prime}$ seeing. Images are $3.3^{\prime}$ on the side. Note the enhanced density of faint sources $(r \gtrsim 23 \mathrm{mag})$ visible in the template image that are not detected in the search image.

shows an $r$-band co-add image of the field around SN2005ir in comparison with a single-exposure search image. Some of our fields near the edge of the stripe fall outside of the co-add, and for these we use single-epoch templates from a night with good seeing.

MATCHTEMPL. Since the SDSS camera is used in drift-scan mode, the data from a given run comprise a long swath of the sky. This tapestry is split by Photo into individual fields of $2048 \times 1489$ pixels, with 128 pixels of overlap between neighboring fields. Because Stripe 82 is an equatorial field, this corresponds to a slice in $\alpha$. It is not guaranteed that the $\alpha$ boundaries of the science image agree exactly with those of the template, meaning, in general, we will need to identify and combine the two template images that overlap the science image. However, the pointing model of the telescope is sufficiently accurate that the overlap in $\delta$ is nearly complete.

GLUETEMPL. This stage implements the pixel-level coaddition of the overlapping template images into a single template image whose R.A. coverage is similar to the input science image.

REMAP. Because the images may still be misaligned in R.A. by a fractional pixel offset, and in declination by multiple pixels, we apply a remapping kernel that exactly aligns the glued template image with the science image. For this operation, we use the astrometry included in the asTrans files to determine, for each pixel in the science image, what position in the template image needs to be resampled. We apply a windowed-sinc resampling of the template image with a $9 \times 9$ pixel footprint (i.e., a Lanczos 4 kernel) to yield a final remapped glued template image. The template is always remapped to the reference frame of the science image, to avoid convolving the science data. Because the template image generally has much higher signal-to-noise (except possibly near the beginning of the stripe where only single-exposure templates are available), the "smoothing" effects due to convolution are not a serious issue. We propagate the pixel noise and pixel masks through this convolution process.

DIFFIM. After the astrometric registration described above, we implement a photometric registration that matches the
PSF of the two images, so that they may be subtracted on a pixel-by-pixel basis. This uses a modified version of the Alard (2000) algorithm as described in Becker et al. (2004).

In this procedure, known objects (stars or galaxies) are used to determine a set of convolution kernels that minimize the difference between each convolved template object and the respective science object. Objects that include masked pixels are rejected, as are variable objects. The ensemble of kernels is then used to construct a single kernel function that varies spatially with position on the image, to account for differences in the PSF across the frames. This function is allowed to vary quadratically across the image. We then apply this kernel to each pixel in the image and subtract the convolved template from the science image, yielding a difference image in which the static signal has been subtracted, and only objects that have varied in position or brightness remain. We choose to always apply this kernel to the template image, even though in good seeing conditions this requires us to actually deconvolve the template data. We propagate the image noise and mask similar to the REMAP process.

DIFFDOPHOT. We next perform object detection on the difference image, again using the modified Dophot package. The software is able to read the propagated noise and mask images to assist in the object detection and measurement. The background is explicitly set to zero, assuming that the difference imaging has produced a background-subtracted image. The Photo PSF model and aperture corrections are used to perform the photometry. We only perform photometry on positive-going excursions, thus objects that fade in brightness are not measured.

PIXCHK. We next read in the difference, noise, and mask images surrounding each detected object and examine the neighboring pixels. The total number of positivevalued pixels, negative-valued pixels, and masked pixels are calculated in an aperture, as well as the total amount of flux in positive-valued and negative-valued pixels. These will be used to reject dipoles due to image misalignment or general failures in the difference imaging.

DIFFCUT. This stage rejects candidates from the photometry list based upon photometry flags and PIXCHK 
values. In particular, objects for which Dophot is unable to perform a 4-parameter fit using the mean PSF parameters are rejected (Dophot types 4 or 6 ). Objects with $\mathrm{S} / \mathrm{N}$ smaller than 3.0 are also rejected, as well as objects with excessive fractions of masked pixels (0.6) or fractions of flux in negative pixels (0.65), amongst other tests. These cuts yield the set of objects ingested into the database by DoObjects.

\section{A4. sdssforce}

This mode is used to perform forced-positional PSF photometry. The coordinates of the candidate are determined from a $\mathrm{S} / \mathrm{N}$-weighted average of all objects associated with the SN. The PSF model is adopted from output of Photo. In practice, this mode is used to measure magnitudes in the $u$ and $z$ bands, which are not automatically processed by sdssdiff, as well as ugriz magnitudes in images obtained before the SN discovery to obtain measurements of low-S/N detections and upper limits. This mode yields more accurate measurements of the fluxes than those produced by sdssdiff, and they generally agree with the final photometry to within $\sim 0.05$ mag in all filters (Holtzman et al. 2007).

\section{APPENDIX B}

\section{AUTOSCANNER}

As a further illustration of the histogram method of PDF estimation, we note that Bayes' theorem, applied to classification, says that

$$
\frac{P\left(c_{i} \mid \theta\right)}{P\left(c_{j} \mid \theta\right)}=\frac{P\left(\theta \mid c_{i}\right) P\left(c_{i}\right)}{P\left(\theta \mid c_{j}\right) P\left(c_{j}\right)}
$$

where $P(a \mid b)$ is the conditional probability for $a$ given $b, c_{i}$ represents object class $i$, and $\theta$ denote particular values for the set of observables of an object. If this ratio is larger than one, then the object is more likely to belong to class $i$ than to class $j$.

Applied to the problem at hand, this can be written as

$$
\frac{P\left(c_{i} \mid \theta\right)}{P\left(c_{j} \mid \theta\right)}=\frac{\left(N_{i}^{\prime} / N_{i}\right)\left(N_{i} / N\right)}{\left(N_{j}^{\prime} / N_{j}\right)\left(N_{j} / N\right)}=\frac{N_{i}^{\prime}}{N_{j}^{\prime}}
$$

where $N_{i}$ is the total number of objects in the training set belonging to class $i, N$ is the number of total objects in the training set, and $\mathrm{a}^{\prime}$ denotes objects possessing observables $\theta$. $\theta$ represents a subset of all possible observables, which is equivalent to marginalizing over all other observables, and the set used differs according to which class of background is being tested.

The criterion used by the autoscanner to identify objects of class $i$ is

$$
\frac{N_{i}^{\prime}}{N_{j}^{\prime}}>\frac{N_{i}}{N_{j}}
$$

where $i$ represents the classes of background (artifact, mover, dipole), and class $j$ is always taken as SN. The motivation for this criterion is that we are searching for an overdensity of objects of class $i$ in the region of observable space in question. In practice, the threshold is a free parameter that can be adjusted to control the relative level of accepted signal to background events.

In this experiment, background events dominate over $\mathrm{SNe}$ epochs by a large factor, and a Bayesian classifier would reject $\mathrm{SN}$ epochs that lie in a reasonable region of the observable
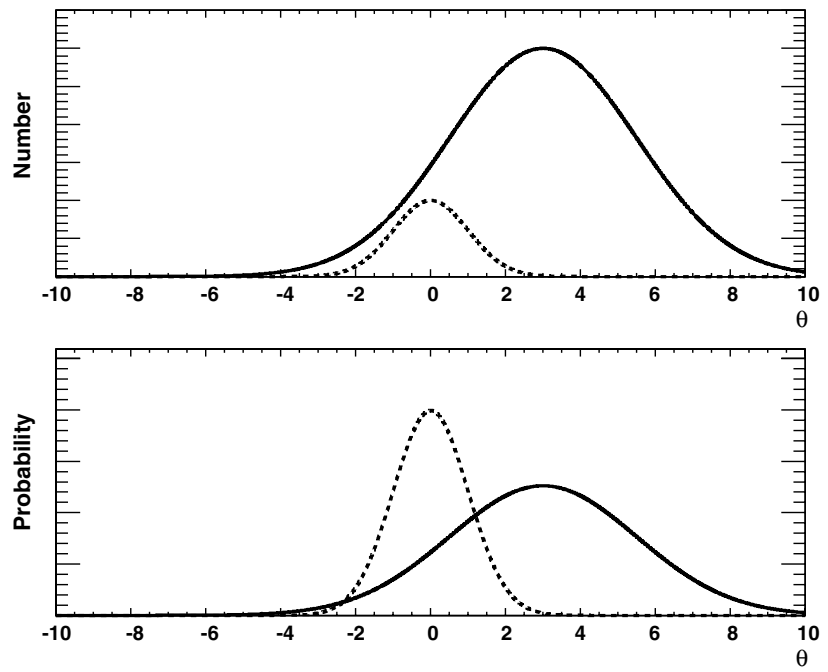

Figure 10. Hypothetical distributions of an observable for signal (dashed) and background (solid). The peak of the signal occurs at 0 ; however, the vastly greater number of background events swamp the signal. While it is true that an object with a value of this observable of 0 is more likely to be a background event, this is clearly not the behavior we want from our classifier, as we are trying to isolate the relatively rare signal events (i.e. SN epochs). The effect of recasting the problem from one of relative probabilities to one of overdensities. The peak of the signal (dashed) is now strongly inconsistent with background.

space if the region overlaps with background events, even far away from the peak of the background distribution. A cartoon of the situation is shown in Figure 10. Thus, the autoscanner can also be regarded as a Bayesian classifier where the thresholds are adjusted $a$ priori to mitigate false negatives, at the cost of more background in the scanning.

The training set for the autoscanner consists of all objects from the 2005 observing season that were ranked by a person as an artifact, a dipole, a moving object, or an SN. This set is comprised of 91,220 total objects, of which 65,576 are a sub-class of background, and 25,644 are objects ranked as an $\mathrm{SN}$. The quantities used for the classification of artifacts are a measure of the object's ellipticity, the $\mathrm{S} / \mathrm{N}$, and the value of the reduced $\chi^{2}$ when fitting the object to a model of the PSF. In computing $\chi^{2}$, we do not account for possible error in the PSF model and so objects with large $\mathrm{S} / \mathrm{N}$ values also have large $c h i^{2}$ values. Therefore, in classifying artifacts it is important to retain the correlation between $c h i^{2}$ and $\mathrm{S} / \mathrm{N}$. The quantities used for classifying dipoles are the measured flux in negative-valued pixels, and the ratio of measured flux in negative-valued pixels to the flux in positive-valued pixels. The quantities used for classifying moving objects are the measured magnitudes, the magnitude of the apparent motion between different filters, and an angle describing the apparent motion between filters. If the object has a detection in the 3 filterbands gri, then the angle is the angle between the "vector" that describes the apparent motion between the $r$ and $i$ filters, and the "vector" that describes the apparent motion between the $i$ and $g$ filters. If the object is truly moving then these apparent motions should be nearly co-linear. If the object is only detected in two of the gri filters then the angle is the angle between the apparent motion between the two filters and the "unit vector" aligned in the direction of increasing right ascension. Main-belt asteroids typically have an apparent motion in a fixed direction, and using this angle in the classification is a way to encode that information. 


\section{APPENDIX C}

\section{SN Ia LIGHT CURVE MODELS}

This appendix describes the SN Ia light curve models used for photometric typing, redshift estimate, and selection of targets for spectroscopic observations. To calculate synthetic light curves of Branch-normal SNe Ia at various values of $\Delta m_{15}(B)$, the $B$-band magnitude change in 15 days from $B$-band maximum (Phillips 1993), we adopt the results of Phillips et al. (1999), who found relationships between the peak absolute $B V I$ magnitudes of nearby $\mathrm{SNe}$ Ia as a function of $\Delta m_{15}(B)$. These authors parameterize the peak magnitude relative to that of a reference SN Ia with $\Delta m(B)=1.1$ using a quadratic function of the form

$$
\Delta M_{\max }=a \times\left[\Delta m_{15}(B)-1.1\right]+b \times\left[\Delta m_{15}(B)-1.1\right]^{2} .
$$

There are $a$ and $b$ coefficients for each of the $B V I$ filters, as listed in Table 3 of Phillips et al. (1999). The $a$ coefficients $\left(a_{B}\right.$ $=0.79, a_{V}=0.67$, and $a_{I}=0.42$ ) decrease as a function of the filter effective wavelength. This shows that SNe Ia with larger $\Delta m_{15}(B)$ are dimmer and redder; those with smaller $\Delta m_{15}(B)$ are intrinsically more luminous and bluer. We find that the $a$ coefficient as a function of wavelength can be well represented by the following linear relation:

$$
a(\lambda)=1.248-1.045 \times 10^{-4} \lambda(\AA) .
$$

We use this relation to perform color corrections directly onto the template spectra. Operationally, defining $F_{v}(\lambda, x=0)$ to be the monochromatic flux ( $\mathrm{erg} \mathrm{cm}^{-2} \mathrm{~s}^{-1} \mathrm{~Hz}^{-1}$ ) of the standard template spectrum at rest-frame wavelength $\lambda$, then the luminosity-corrected value is given by

$$
F_{v}(\lambda, x) \longrightarrow F_{v}(\lambda, x=0) \times 10^{-0.4\left(a x+b x^{2}\right)},
$$

where $x \equiv \Delta m_{15}(B)-1.1, b=0.633$ (Phillips et al. 1999), and $a$ is given in Equation (C2). Although this color correction is valid at $B$-band maximum, we assume that it is valid at all epochs. This provides a way to compute synthetic light curves in the ugriz system (or any other filter set) for a SN Ia with a given $\Delta m_{15}(B)$ at arbitrary redshifts.

Finally, we stretch the light curves according to the method described in Perlmutter et al. (1997). The Nugent templates folded through the $B$-band filter transmission curve yield $\Delta m_{15}(B)=1.05$, so we define $s$ to be unity when $\Delta m_{15}(B)=$ 1.05. For other values of $\Delta m_{15}(B)$, we express the stretch factor as $s=(1+z)(15 / \tau)$, where we have factored out the cosmological time delay factor, and $\tau$ is represented by the following third-order polynomial:

$\tau[\Delta m(B)]=c_{0}+c_{1} \Delta m_{15}(B)+c_{2}\left[\Delta m_{15}(B)\right]^{2}+c_{3}\left[\Delta m_{15}(B)\right]^{3}$,

where $c_{0}=3.455, c_{1}=13.719, c_{2}=-3.601$, and $c_{3}=0.946$. This gives an adequate representation of the $B$-band light curve for $\Delta m_{15}(B)$ between 0.5 and 2.5. This function $s\left[\Delta m_{15}(B)\right]$ at zero redshift is plotted in Figure 11. The same relation derived by Perlmutter et al. (1997) is also shown for comparison. Figure 12 shows rest-frame $g$-band light curves for different values of $\Delta m_{15}(B)$. Peak absolute magnitudes in ugriz as a function of $\Delta m_{15}(B)$ are shown in Figure 13.

Although this procedure does not always accurately reproduce the multi-band light curves, most notably the variation of the secondary peak in the red with $\Delta m_{15}(B)$ (Riess et al. 1996),

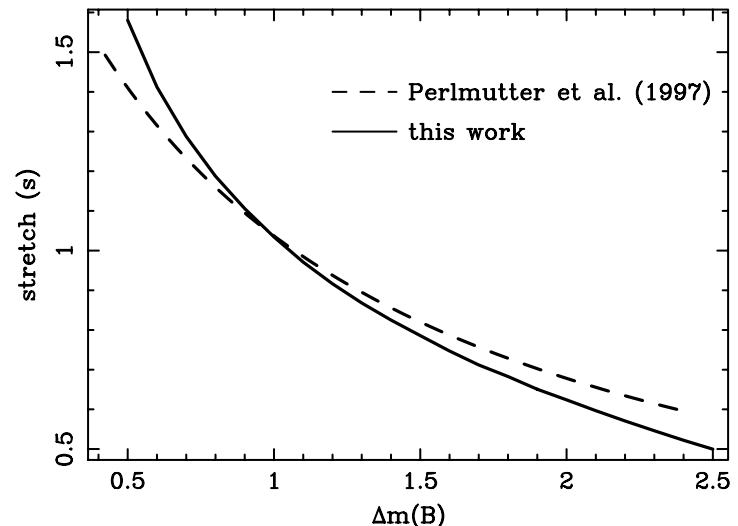

Figure 11. The stretch factor $s$ at $z=0$ as a function of $\Delta m_{15}(B)$ adopted in this work (black) in comparison to that of Perlmutter et al. (1997) represented by a dashed line.

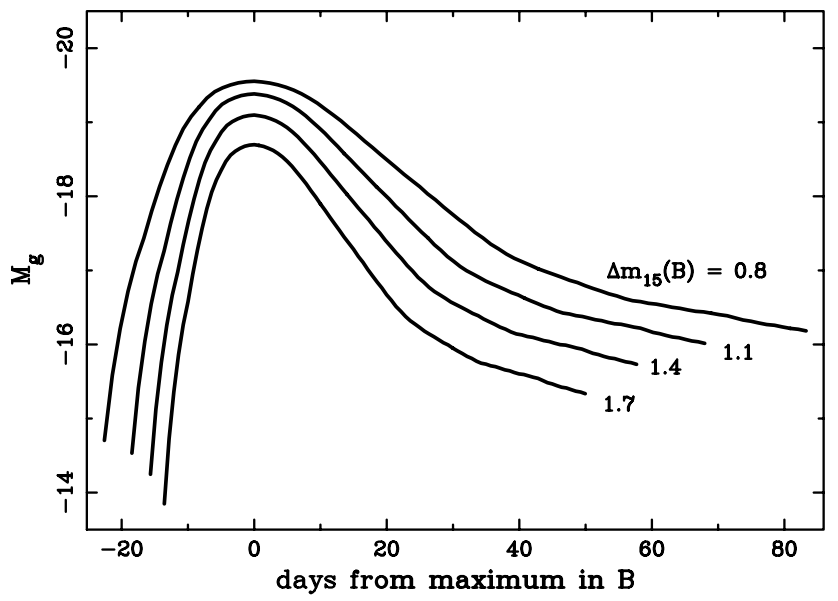

Figure 12. Sample SN Ia rest-frame $g$-band light curves for four values of $\Delta m_{15}(B)=0.8,1.1,1.4$, and 1.7 .

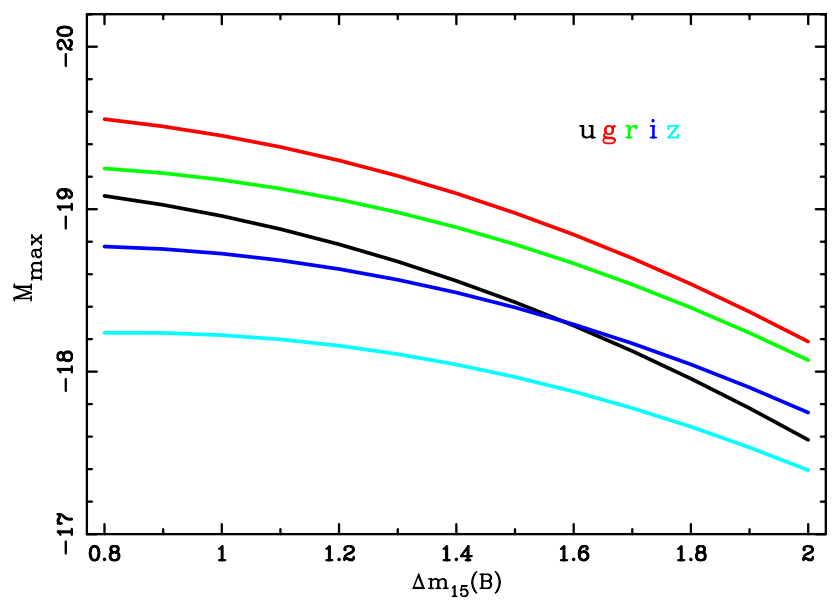

Figure 13. Resulting peak absolute magnitudes for a SN Ia at zero redshift in the SDSS ugriz system as functions of $\Delta m_{15}(B)$. The curves are analogous to those shown in Figure 8 of Phillips et al. (1999).

(A color version of this figure is available in the online journal)

they appear to be of sufficient quality for the purposes of photometric typing. 


\section{REFERENCES}

Adelman-McCarthy, J., et al. 2007, ApJS, in press

Adelman-McCarthy, J., et al. 2007, ApJS, submitted

Adelman-McCarthy, J. K., et al. 2006, ApJS, 162, 38

Alard, C. 2000, A\&AS, 144, 363

Aldering, G., et al. 2002, Proc. SPIE, 4836, 61

Annis, J. T., et al. 2006, American Astronomical Society Meeting Abstracts, 209, \#215.09

Astier, P., et al. 2006, A\&A, 447, 31

Barbary, K., et al. 2006, Bull. Am. Astron. Soc., 38, 1025

Becker, A. C., et al. 2004, ApJ, 611, 418

Dilday, B., et al. 2007, in preparation

Filippenko, A. V. 1997, ARA\&A, 35, 309

Filippenko, A. V., Li, W. D., Treffers, R. R., \& Modjaz, M. 2001, IAU Colloq. 183: Small Telescope Astronomy on Global Scales, 246, 121

Frieman, J., et al. 2008, AJ, 135, 338

Fukugita, M., Ichikawa, T., Gunn, J. E., Doi, M., Shimasaku, K., \& Schneider, D. P. 1996, AJ, 111, 1748

Gunn, J. E., et al. 1998, AJ, 116, 3040

Gunn, J. E., et al. 2006, AJ, 131, 2332

Hamuy, M., Phillips, M. M., Suntzeff, N. B., Schommer, R. A., Maza, J., \& Aviles, R. 1996, AJ, 112, 2391

Hamuy, M., Phillips, M. M., Suntzeff, N. B., Schommer, R. A., Maza, J., \& Aviles, R. 1996, AJ, 112, 2398

Hamuy, M., Phillips, M. M., Suntzeff, N. B., Schommer, R. A., Maza, J., Smith, R. C., Lira, P., \& Aviles, R. 1996, AJ, 112, 2438

Hamuy, M., et al. 1993, AJ, 106, 2392

Hamuy, M., et al. 1996, AJ, 112, 2408

Hamuy, M., et al. 2006, PASP, 118, 2

Hogg, D. W., Finkbeiner, D. P., Schlegel, D. J., \& Gunn, J. E. 2001, AJ, 122,2129

Holtzman, J., et al. 2007, in preparation

Hsiao, E. Y., Conley, A., Howell, D. A., Sullivan, M., Pritchet, C. J., Carlberg, R. G., Nugent, P. E., \& Phillips, M. M. 2007, ApJ, 663,1187

Ivezić, Ž., Allyn Smith, J., Miknaitis, G., Lin, H., \& Tucker, D. 2007, arXiv astro-ph/0703157

Ivezić, Ž., et al. 2004, Astron. Nachr., 325, 583

Jha, S., Riess, A. G., \& Kirshner, R. P. 2007, ApJ, 659, 122

Jha, S., et al. 2006, AJ, 131, 527

Johnson, B. D., \& Crotts, A. P. S. 2006, AJ, 132, 756

Kasen, D., \& Woosley, S. E. 2007, ApJ, 656, 661

Kessler, R., et al. 2007, in preparation

Kim, A., Goobar, A., \& Perlmutter, S. 1996, PASP, 108, 190

Kunz, M., Bassett, B. A., \& Hlozek, R. A. 2007, Phys. Rev. D, 75, 103508
Kuznetsova, N. V., \& Connolly, B. M. 2007, ApJ, 659, 530

Lupton, R., Gunn, J. E., Ivezić, Z., Knapp, G. R., \& Kent, S. 2001, Astron. Data Anal. Softw. Syst. X, 238, 269

Lupton, R. H., Ivezic, Z., Gunn, J. E., Knapp, G., Strauss, M. A., \& Yasuda, N. 2002, Proc. SPIE, 4836, 350

Miknaitis, G., et al. 2007, arXiv astro-ph/0701043

Nugent, P., Kim, A., \& Perlmutter, S. 2002, PASP, 114, 803

Oyaizu, H., et al. 2007, in preparation

Perlmutter, S., et al. 1997, ApJ, 483, 565

Perlmutter, S., et al. 1999, ApJ, 517, 565

Pier, J. R., Munn, J. A., Hindsley, R. B., Hennessy, G. S., Kent, S. M., Lupton, R. H., \& Ivezić, Ž. 2003, AJ, 125, 1559

Phillips, M. M. 1993, ApJ, 413, L105

Phillips, M. M., Lira, P., Suntzeff, N. B., Schommer, R. A., Hamuy, M., \& Maza, J. 1999, AJ, 118, 1766

Phillips, M. M., et al. 2007, PASP, 119, 360

Poznanski, D., Gal-Yam, A., Maoz, D., Filippenko, A. V., Leonard, D. C., \& Matheson, T. 2002, PASP, 114, 833

Poznanski, D., Maoz, D., \& Gal-Yam, A. 2006, arXiv astro-ph/0610129

Prieto, J., Anderson, S., Becker, A., Marriner, J., Sako, M., \& Jha, S. 2006, Cent. Bur. Electron. Telegrams, 692, 1

Prieto, J. L., et al. 2007, in preparation

Pskovskii, I. P. 1977, Sov. Astron., 21, 675

Quimby, R., Höflich, P., \& Wheeler, J. C. 2007, arXiv astro-ph/0705.4467, 705 Riess, A. G. 1996, PhD thesis

Riess, A. G., Press, W. H., \& Kirshner, R. P. 1995, ApJ, 438, L17

Riess, A. G., Press, W. H., \& Kirshner, R. P. 1996, ApJ, 473, 88

Riess, A. G., et al. 1998, AJ, 116, 1009

Riess, A. G., et al. 1999, AJ, 117, 707

Riess, A. G., et al. 2004a, ApJ, 607, 665

Riess, A. G., et al. 2004b, ApJ, 600, L163

Riess, A. G., et al. 2007, ApJ, 659, 98

Sako, M., et al. 2005, in Proceedings of the 22nd Texas Symposium on

Relativistic Astrophysics at Stanford, 2004, ed. P. Chen, E. Bloom,

G. Madejski, \& V. Petrosian eConf C041213, 1424

Schechter, P. L., Mateo, M., \& Saha, A. 1993, PASP, 105, 1342

Shetrone, M., et al. 2007, PASP, 119, 556

Smith, C., Rest, A., Hiriart, R., Becker, A., Stubbs, C. W., Valdes, F. G., \& Suntzeff, N. 2002, Proc. SPIE, 4836, 395

Smith, J. A., et al. 2002, AJ, 123, 2121

Stoughton, C., et al. 2002, AJ, 123, 485

Sullivan, M., et al. 2006, AJ, 131, 960

Tucker, D. L., et al. 2006, Astron. Nachr., 327, 821

Wood-Vasey, W. M., et al. 2007, arXiv astro-ph/0701041

York, D. G., et al. 2000, AJ, 120, 1579

Zheng, C., et al. 2007, in preparation 